\title{
Parental-fetal interplay of immune genes leads to intrauterine growth restriction
}

Gurman Kaur ${ }^{1,2,16}$, Caroline B. M. Porter ${ }^{2,16}$, Orr Ashenberg ${ }^{2}$, Jack Lee ${ }^{3}$, Samantha J. Riesenfeld ${ }^{2,4}$, Matan Hofree ${ }^{2}$, Maria Aggelakopoulou ${ }^{5}$, Ayshwarya Subramanian², Subita Balaram Kuttikkatte ${ }^{1}$, Kathrine E. Attfield ${ }^{5}$, Christiane A. E. Desel ${ }^{5,6}$, Jessica L. Davies ${ }^{5}$, Hayley G. Evans ${ }^{5}$, Inbal AvrahamDavidi $^{2}$, Lan T. Nguyen², Danielle A. Dionne ${ }^{2}$, Anna E. Neumann ${ }^{7}$, Lise Torp Jensen ${ }^{8}$, Thomas R. Barber $^{1}$, Elizabeth Soilleux ${ }^{9}$, Mary Carrington ${ }^{10,11}$, Gil McVean ${ }^{12}$, Orit Rozenblatt-Rosen ${ }^{2,13}$, Aviv Regev $^{2,13,14,15, *}$, Lars Fugger ${ }^{1,5,8, *}$

${ }^{1}$ MRC Human Immunology Unit, MRC Weatherall Institute of Molecular Medicine, John Radcliffe Hospital, University of Oxford, Oxford, UK

${ }^{2}$ Klarman Cell Observatory, Broad Institute of MIT and Harvard, Cambridge, MA, USA

${ }^{3}$ Department of Biomedical Engineering, School of Biomedical Engineering and Imaging Sciences, King's

College London, London, UK

${ }^{4}$ Pritzker School of Molecular Engineering, University of Chicago, Chicago, IL, USA

${ }^{5}$ Oxford Centre for Neuroinflammation, Nuffield Department of Clinical Neurosciences, MRC Weatherall Institute of Molecular Medicine, John Radcliffe Hospital, University of Oxford, Oxford, UK

${ }^{6}$ Current address: University Department of Neurology, University Hospital Magdeburg, Germany

${ }^{7}$ Broad Institute of MIT and Harvard, Cambridge, MA, USA

${ }^{8}$ Department of Clinical Medicine, Aarhus University Hospital, Aarhus, Denmark

${ }^{9}$ Department of Pathology, Tennis Court Rd, University of Cambridge

${ }^{10}$ Basic Science Program, Frederick National Laboratory for Cancer Research in the Laboratory of Integrative Cancer Immunology, National Cancer Institute, Bethesda, MD USA

${ }^{11}$ Ragon Institute of MGH, MIT, and Harvard, Cambridge, MA USA

${ }^{12}$ Big Data Institute, Li Ka Shing Centre for Health Information and Discovery, University of Oxford, Oxford, UK

${ }^{13}$ Current address: Genentech, 1 DNA Way, South San Francisco, CA, USA

${ }^{14}$ Massachusetts Institute of Technology, Department of Biology, Cambridge, MA, USA

${ }^{15}$ Howard Hughes Medical Institute, Chevy Chase, MD, USA

${ }^{16}$ These authors contributed equally

*Correspondence: lars.fugger@ndcn.ox.ac.uk (L.F.), aregev@broadinstitute.org (A.R.) 


\begin{abstract}
Intrauterine growth restriction (IUGR) of fetuses affects $5-10 \%$ of pregnancies and is associated with perinatal morbidity, mortality and long-term health issues. Understanding genetic predisposition to IUGR is challenging, owing to extensive gene polymorphisms, linkage disequilibrium, and maternal and paternal influence. Here, we demonstrate that the inhibitory receptor, KIR2DL1, expressed on maternal uterine natural killer ( $\mathrm{uNK}$ ) cells, in interaction with the paternally-inherited HLA-C*05, an HLA-C group 2 allotype, expressed on fetal trophoblast cells, causes IUGR in a humanised mouse model. Micro-CT imaging of the uteroplacental vasculature revealed reduced uterine spiral artery diameter and increased segment length, increasing fetal blood flow resistance. Single cell RNA-Seq from the maternal-fetal interface highlighted expression programs activated by KIR2DL1-induced IUGR in several placental cell types, including degradation of extracellular matrix components, angiogenesis, and uNK cell communication, suggesting a complex response underlying IUGR. As current IUGR treatments are insufficient, our findings provide important insight for drug development.
\end{abstract}




\section{INTRODUCTION}

Intrauterine growth restriction (IUGR) refers to pathologically reduced fetal growth and occurs in about $5-10 \%$ of pregnancies in developed countries, with higher proportions in developing countries (de Onis et al., 1998; Imdad and Bhutta, 2013; Romo et al., 2009). IUGR is one of the largest contributors to perinatal mortality and morbidity (Bernstein et al., 2000; Ego et al., 2013; Gardosi et al., 2005; Moraitis et al., 2014; Resnik, 2002; Rosenberg, 2008), and predisposes later in life to heart disease, hypertension, type 2 diabetes and stroke (Forsen et al., 2000; Frankel et al., 1996; Horikoshi et al., 2016; Leon et al., 1998; Rich-Edwards et al., 1997; Tyrrell et al., 2013; Warrington et al., 2019). IUGR can occur alone or with pre-eclampsia, a serious and systemic pregnancy complication characterised by maternal newonset hypertension and proteinuria (Burton and Jauniaux, 2018; Steegers et al., 2010).

Despite their serious clinical implications, the causes, disease mechanisms and relationship of IUGR and pre-eclampsia are poorly understood, and effective prevention and treatment strategies are lacking (Figueras and Gardosi, 2011; McCowan et al., 2018; Nardozza et al., 2017). This is partly because studying IUGR and pre-eclampsia in human pregnancies is logistically challenging, due to the inaccessibility of tissue during critical early stages of pregnancy. Thus, there is an unmet need to better understand disease pathogenesis to identify diagnostic and therapeutic tools that improve maternal and fetal health, and long-term health of the child.

IUGR risk factors can be fetal, maternal or placental in origin. Pathogenesis is suspected to occur early in placentation when the definitive placenta is forming, and lie in placental insufficiency and dysfunction, such as higher uterine resistance to blood flow (Burton and Jauniaux, 2018; Li et al., 2018; Malhotra et al., 2019; Nardozza et al., 2017). Genetic association studies in humans suggest that uterine natural killer (uNK) cell surface receptors and fetal trophoblast ligands play a role in controlling placentation. uNK cells express a combination of inhibitory and activating killer cell immunoglobulinlike receptors (KIRs) on their cell surface, are the most abundant lymphocytes at the maternal-fetal interface, especially during early to mid-gestation, and are implicated in placental vascular remodeling (Ashkar et al., 2003; Ashkar et al., 2000; Bashirova et al., 2006; Hanna et al., 2006; Hiby et al., 2010; 
Lima et al., 2012; Sojka et al., 2018). HLA-C alleles are KIR ligands and can modulate NK cell function. HLA-C is the only polymorphic HLA molecule expressed on the surface of fetal extravillous trophoblasts (cells derived from the outer layer of the blastocyst, defining the boundary between the mother and the fetus) and there is direct contact between $\mathrm{uNK}$ and trophoblast cells during placentation (Apps et al., 2009; King et al., 2000; Lanier, 2005; Moffett and Loke, 2006).

Pregnant women with two copies of the KIR A haplotype (i.e., KIR AA), composed of inhibitory KIR genes, have a higher risk (odds ratio 1.32-1.93) of pregnancy complications such as IUGR, preeclampsia and recurrent miscarriage, especially if the fetus inherits a group 2 HLA-C allele from the father (odds ratio 2.02). In contrast, the KIR B haplotype, mostly composed of activating KIR genes, is associated with protection against pregnancy disorders and high birth weight (Hiby et al., 2014; Hiby et al., 2010; Hiby et al., 2004; Nakimuli et al., 2015). KIR and HLA genes are highly polymorphic and inherited in complex haplotypes (Bashirova et al., 2006; Lanier, 2005; Parham, 2005), making it genetically challenging to ascertain the role of specific genes in disease pathogenesis. A typical KIR A haplotype consists of seven KIR genes and two pseudogenes, with extensive allelic variation present at several of these genes. Due to its strict binding specificity to group 2 HLA-C alleles, KIR2DL1 represents a candidate gene likely to confer the genetic risk observed in pregnancy complications (Bashirova et al., 2006; Hiby et al., 2004). Nevertheless, the KIR haplotypes have extensive gene polymorphism and strong linkage disequilibrium, so determining the role of specific genes requires teasing their effects apart from the haplotype by testing with functional assays.

Recent studies have used single cell RNA-seq (scRNA-seq) to begin to shed light on the complex cellular composition of the maternal fetal interface. In particular profiling of total immune (CD45+) cells from healthy human pregnancies (Vento-Tormo et al., 2018), highlighted three major subsets of NK cells in first trimester deciduas from healthy pregnant women, all of which expressed tissue-resident markers, but differed in their immunomodulatory profiles. Another study profiled cells from healthy and early pre-eclamptic placentas at the point of delivery (Tsang et al., 2017), but did not specifically highlight populations of NK cells. By necessity, however, such studies could only explore either first 
trimester placentas and be focused on likely healthy pregnancies, rather than the context of IUGR and relevant defined genotypes, or study placentas at the time of delivery by which point the cellular composition of the tissue is significantly different from that of early/mid-gestation.

Here, we developed a novel humanised mouse model system in which the interaction between the inhibitory KIR2DL1 receptor on maternal uNK cells and paternally-derived HLA-C*05, an HLA-C group 2 allotype, on fetal trophoblast cells leads to IUGR in fetuses. Our system highlights the involvement of this specific KIR-HLA-C interaction in disease pathogenesis, allowing us to study its role at the maternal-fetal interface in pregnancy. We show that this interaction leads to an increase in uterine vascular resistance, evidenced by a reduction in uterine spiral artery radius and an increase in arterial segment length. We performed single cell RNA-seq (scRNA-seq) on sorted populations of uNK cells from healthy and IUGR pregnancies to build a uNK cell atlas from the maternal-fetal interface at mid-gestation, revealing seven functionally heterogenous uNK subtypes. Topic modeling with Latent Direchelet Allocation (LDA) highlighted gene programs that are significantly perturbed in IUGR, including a program with Gzmd, Gzme and Gzmg expression in tissue-resident uNK cells, and a program involved in lymphocyte activation in circulating uNK cells. Further scRNA-seq of unsorted cells at the maternal-fetal interface at mid-gestation revealed expression changes in the IUGR phenotype for several non-NK cell types and a change in cellular communication between these cell types and uNK cells, suggesting a coordinated response leads to IUGR manifestation in pregnancy.

\section{RESULTS}

\section{A humanised HLA-C*05 and KIR2DL1 transgenic mouse model}

To characterise the role of specific KIR and HLA-C genes at the maternal-fetal interface, we used KIR2DL1 from the KIR A haplotype due to its strict specificity to bind group 2 HLA-C alleles expressed on fetal trophoblast cells. We chose KIR2DL1*0030201 because it is a common KIR2DL1 inhibitory allele typically found on the KIR A haplotype. Correspondingly, we chose HLA-C*05 (HLA$C^{*}$ 05:01:01:01), a common group 2 HLA-C allele. We developed humanised single transgenic mice 
with physiological expression of HLA-C*05 or KIR2DL1 and double transgenic mice expressing both HLA-C*05 and KIR2DL1.

The HLA-C*05 transgenic mice expressed HLA-C*05 on all MHC-class I expressing cells as HLA$\mathrm{C}^{*} 05$ was driven by the endogenous $H L A-C^{*} 05$ promoter. To facilitate interaction of the HLA-class I molecule with murine CD8 (and hence the T cell receptor), we replaced the $\alpha 3$ domain of HLA-C*05 with its murine counterpart from $\mathrm{H} 2-\mathrm{K}^{\mathrm{b}}$, along with the adjacent transmembrane and cytoplasmic domains - as has been done for other HLA-Class I genes (Borenstein et al., 2000) (Fig. 1a). We confirmed that HLA-C*05 was expressed in all cells of all tested organs using an anti-HLA-C antibody and qPCR primers specific for HLA-C (Fig. 1b and S1a). We further confirmed that different populations of immune cells in the spleen or thymus, as well as thymic epithelial cells (TECs), expressed HLA-C*05 (Fig. 1c,d), demonstrating physiological expression of the transgene in the mouse model. HLA-C*05-expressing splenocytes responded to in vitro stimuli such as IFN $\gamma$ or LPS by upregulating HLA-C expression (Fig. 1e,f and S1b, c), in agreement with previous observations (Otsuka et al., 1991).

To model the fact that KIR genes are predominantly expressed on NK cells in humans (Bashirova et al., 2006; Vilches and Parham, 2002), we generated mice where KIR2DL1 is restricted to murine NK cells. To this end, we generated transgenic mice with a loxP flanked stop cassette between the KIR2DL1 coding DNA sequence and CAG promoter, and mated them to mice expressing iCre-recombinase in cells expressing the NK cell marker, Ncrl (i.e., Ncrl-iCre BAC transgenic mice) (Eckelhart et al., 2011) (Fig. 1g), to generate KIR2DL1-expressing mice. Staining immune cells using anti-KIR antibody demonstrated specific and high expression of KIR2DL1 on NK cells only, with expression in $~ 75-90 \%$ of NK cells (Fig. 1h,i).

Expression of KIR2DL1 or HLA-C*05 did not alter the proportion of NK cells (Fig. S1d) or other immune cell types (Fig. S1e,f) in the lymphoid compartments of the transgenic mice expressing HLA- 
$\mathrm{C}^{*} 05$, KIR2DL1 or both HLA-C*05 and KIR2DL1, and NK cell receptor and transcription factor expression essential for mouse NK cell development remained unaltered (Fig. S1g-i). To minimize perturbations introduced to the transgenic model system, we did not knockout any mouse MHC class I or NK cell receptor molecules or abrogated any immune cell types. We further confirmed that NK cell development and maturation was normal and comparable to that observed in wild-type (WT) littermates by testing CD27 and CD11b expression, genes that represent distinct NK-cell development stages (Chiossone et al., 2009) (Fig. 1j,k and S1j,k).

In summary, to test the hypothesis that KIR2DL1 and HLA-C*05 in combination play a pathogenic role in the development of IUGR, we developed a novel humanised transgenic model system with specific expression of the inhibitory KIR2DL1 receptor on murine NK cells and the corresponding expression of the KIR2DL1 ligand, HLA-C*05, on all MHC-class I expressing cells in mice.

\section{KIR2DL1 and HLA-C*05 are functional in the transgenic mice}

We next validated that both KIR2DL1 and HLA-C*05 were functional in the transgenic setting. First, to test if signalling via the inhibitory KIR2DL1 receptor impacted NK cell responses, we stimulated splenocytes from WT, HLA-C*05- or KIR2DL1-expressing transgenic mice in vitro using antibodies that activate NK cell receptors (NKp46 or Ly49D), observing the expected increase in IFN $\gamma$ production by NK cells. Presence of $\alpha$-KIR2DL1 antibody along with $\alpha$-NKp46 or $\alpha$-Ly49D led to significant decrease (p-value $<0.05$, Mann-Whitney test) in IFN $\gamma$ production in KIR2DL1-expressing splenocytes, but not in HLA-C*05 or WT splenocytes (Fig. 2a,b), confirming inhibitory signalling via the KIR2DL1 transgene.

We also assessed KIR2DL1 binding by HLA-C*05 in vitro by incubating the KIR2DL1-expressing NK cell line (YT-KIR2DL1) with 721.221-C*05 transfectants and quantifying intracellular IFN $\gamma$ to measure NK cell activation. Co-culture with 721.221-C*05 transfectants, but not the control 721.221-vector or 721.221-C*07 transfectants, dampened the production of IFN $\gamma$ by KIR2DL1-expressing NK cell line, 
confirming that HLA-C*05 (but not HLA-C*07 or the empty vector transduced cells) was bound by KIR2DL1 (Fig. S11).

Next, to assess whether the KIR2DL1 transgene conferred inhibitory signals to NK cells upon recognition of HLA-C*05 in vivo, we performed adoptive transfer experiments. We isolated splenocytes from either knockout mice of murine MHC class I molecules, $\mathrm{H}_{2} \mathrm{~K}^{\mathrm{b}}$ and $\mathrm{H}_{2} \mathrm{D}^{\mathrm{b}}$ (" $\mathrm{KO}$ cells"), WT mice (normal expression of $\mathrm{H}_{2} \mathrm{~K}^{\mathrm{b}}$ and" H2D" "WT cells") or HLA-C*05 transgenic mice bred to $\mathrm{H}_{2} \mathrm{~K}^{\mathrm{b}}$ and $\mathrm{H} 2 \mathrm{D}^{\mathrm{b}} \mathrm{KO}$ ("HLA-C* $05^{+} \mathrm{KO}$ cells"). We labelled KO, WT and HLA-C* $05^{+} \mathrm{KO}$ splenocytes with different concentrations of the florescent cell labelling dye CFSE to distinguish them, and injected them into either WT or KIR2DL1-expressing transgenic mice. As expected, KO cells showed increased rejection in both WT or KIR2DL1-expressing recipient mice due to missing-self recognition, given the capacity of NK cells to attack cells that do not express sufficient levels of MHC class I molecules of the host (Karre et al., 1986). The HLA-C* $05^{+} \mathrm{KO}$ cells were protected from NKcell mediated rejection in KIR2DL1-expressing mice (but not WT mice) (p-value $<0.001$, MannWhitney test) (Fig. 2c-e), showing that HLA-C*05 engagement by the KIR2DL1-expressing NK cells repressed mouse NK cell activating pathways.

\section{KIR2DL1 and HLA-C*05 are expressed at the maternal-fetal interface}

We next evaluated the expression of the KIR2DL1 and HLA-C*05 transgenes at the maternal-fetal interface in two different mating combinations: (1) KIR2DL1-expressing female mated with a HLA$\mathrm{C}^{*} 05$ expressing male (KIR $\times \mathrm{C}^{*} 05$ ), chosen because in humans there is a higher risk of IUGR when the mother has the KIR A haplotype (always carrying KIR2DL1) and the father contributes the group2 HLA-C allele (Hiby et al., 2014; Hiby et al., 2010; Hiby et al., 2004; Nakimuli et al., 2015); and (2) WT female mated with a HLA-C*05 expressing male (WT x $\mathrm{C}^{*} 05$ ), as a control. HLA-C*05 mating males were homozygous for HLA-C*05, such that all fetuses in the litter would have the paternallyderived HLA-C*05 allele. Because the frequency of uNK cells peaks at early to mid-gestation and declines in late pregnancy (Lima et al., 2012; Sojka et al., 2018), we isolated uNK cells from mid- 
gestation (gestation day (gd) 9.5) from the maternal-fetal interface of KIR x C*05 and WT x C*05 matings.

In KIR x C*05, KIR2DL1 was expressed in $\sim 85-90 \%$ of all uNK cells-including $\mathrm{CD}^{*} 9^{+}$or DX $5^{+}$cell subsets, known to distinguish tissue resident (trNK) and conventional (cNK) NK cells (cNK cells circulate in the blood and spleen), respectively (Sojka et al., 2014); no KIR staining was observed in WT x C*05 (Fig. 2f,g). A third control, where a double transgenic female expressing HLA-C*05 and KIR2DL1 was mated with a WT male (C*05/KIR x WT), also expressed KIR in $\sim 85-90 \%$ of all uNK cells (Fig. 2g). The proportion of total uNK cells or $\mathrm{CD} 49 \mathrm{a}^{+}$and $\mathrm{DX}^{+}$uNK cell subsets was comparable across mating combinations (Fig. S1 m,n).

We assessed expression of HLA-C*05 at the maternal-fetal interface in uterine tissue isolated from implantation sites at gd9.5 or gd10.5 of an HLA-C*05 negative female mated to either an HLA-C*05 positive or HLA-C*05 negative male. This meant that HLA-C*05 expression at the maternal-fetal interface could be ascribed only to the paternally-derived HLA-C*05 allele on fetal trophoblast cells. We detected HLA-C mRNA expression in $\mathrm{C}^{*} 05^{+}$but not in $\mathrm{C}^{*} 05^{-}$fetal cells (Fig. $2 \mathbf{h}$ ), and HLA-C DNA, by genotyping of the fetus using genomic DNA isolated from embryonic tissue. Furthermore, we isolated and cultured fetal trophoblast cells from gd12.5 placentas and validated the paternally-derived HLA-C expression in HLA-C* $05^{+}$trophoblast cells by flow cytometry. Cultured fetal trophoblast cells were predominantly $\mathrm{CD} 45^{-}$Cytokeratin $-7^{+}$(data not shown), and in vitro stimulation with LPS + IFN $\gamma$ led to a significant upregulation of HLA-C expression in HLA-C* $05^{+}$(p-value $<0.01$, Mann-Whitney test), but not HLA-C*05, trophoblast cells (Fig. 2i,j, - — as shown previously (King et al., 2000).

Taken together, these results demonstrated the functionality of the KIR2DL1 and HLA-C*05 transgenes in the mouse model system and their expression at the maternal-fetal interface, confirming the validity of this model to study IUGR-relevant disease mechanisms. 


\section{Maternal KIR2DL1 and paternal HLA-C*05 expression leads to IUGR in fetuses}

Because birth and fetal weights are markers for intrauterine development, we investigated the effect of the KIR-HLA-C interactions in mediating IUGR by assessing fetal weight in the three mating combinations described above (KIR $\times \mathrm{C}^{*} 05$, WT $\mathrm{x} \mathrm{C}^{*} 05$ and $\mathrm{C}^{*} 05 / \mathrm{KIR} \times \mathrm{WT}$ ), as well as two additional controls (KIR x WT and WT x WT). Weight was measured just before birth, at gd18.5, to avoid milk uptake as a confounding factor.

Pregnancies involving females with KIR2DL1 expression on uNK cells and males with expression of HLA-C*05 (KIR x C*05 mating) led to IUGR in the fetuses, evidenced by a reduction in the average fetal weight of the embryos compared to all other mating combinations. The progeny from the KIR $\mathrm{x}$ $C^{*} 05$ mating were $\sim 12 \%$ lower in weight compared to the control WT x $C^{*} 05$ mating (Fig. 3a, p-value $<10^{-3}$, linear mixed-effects model) and $\sim 56 \%$ of KIR $\times \mathrm{C}^{*} 05$ fetuses were below the $10^{\text {th }}$ weight percentile of control WT $\times C^{*} 05$ fetuses (with $36 \%$ below the $5^{\text {th }}$ percentile of control WT $\times C^{*} 05$ fetuses) (Fig. 3b). The placental weight was very similar across mating combinations (Fig. 3c), concordant with the lack of observed lesions or pathology in the placenta assessed by H\&E staining (data not shown). The observed $12 \%$ reduction in average fetal weight and the skewed fetal weight distribution of the KIR x C*05 fetuses mirrors clinical observations (Figueras and Gardosi, 2011; Hiby et al., 2014; Moraitis et al., 2014).

Pregnant KIR2DL1-expressing females from the KIR x $C^{*} 05$ mating did not show evidence of hypertension throughout the course of gestation and post-partum (Fig. S2a) or of proteinuria (Fig. S2b), both hallmarks of pre-eclampsia (Steegers et al., 2010). Increased levels of the anti-angiogenic molecule soluble fms-like tyrosine kinase 1 (sFLT-1) and reduced levels of placental growth factor (PIGF) are associated with an increased risk of developing pre-eclampsia in suspected pregnancies (Duhig et al., 2019; Levine et al., 2004; Zeisler et al., 2016), however, their usability in prediction of IUGR is limited (Conde-Agudelo et al., 2013). We did not observe any change in the circulating levels of sFLT-1, soluble Endoglin, or PIGF in the plasma of pregnant females from the KIR $\mathrm{x} \mathrm{C}^{*} 05$ mating combination (Fig. S2c-e). 
Collectively, these results demonstrate the impact of specific KIR and HLA genes on IUGR, with direct evidence of the involvement of KIR2DL1 and HLA-C*05 genes. Furthermore, they corroborate and elaborate on human genetic association studies showing that there was a significant increase in fetal growth restriction, pre-eclampsia and recurrent miscarriage in pregnancies in women with two copies of the KIR A haplotype, but only when there was either a paternally-inherited group 2 HLA-C gene in the fetus, or when the fetus had more group 2 HLA-C genes than the mother (Hiby et al., 2010). Moreover, the impact we observed on IUGR in the fetuses was not associated with any manifestations of a systemic disease such as pre-eclampsia. This could suggest that that additional risk factors or mechanisms are required for pre-eclampsia manifestation.

\section{Maternal KIR2DL1 and paternal HLA-C $* 05$ expression leads to changes in uterine spiral arteries during gestation}

Because compromised uterine circulation may contribute to IUGR (Burton and Jauniaux, 2018), we looked for changes in maternal uterine spiral arteries that feed the developing fetus in the KIR $\mathrm{x} \mathrm{C}^{*} 05$ mice at gd10.5, a point when coiling spiral arteries in the decidua have formed (Adamson et al., 2002). Mice were perfused with an X-ray opaque contrast agent into the uteroplacental circulation and imaged using micro-computed tomography (micro-CT) to preserve the in vivo morphology of the circulatory spaces (Fig. 4a). We manually segmented the spiral arteries in both the KIR x C*05 (IUGR) and WT x $\mathrm{C}^{*} 05$ (control) mating combinations (Fig. 4b,c) and skeletonized them with homotopic thinning (Fig. S3a,b).

The mean diameter of the spiral arteries was reduced by $\sim 15 \%$ in the KIR x C*05 IUGR mice $v s$. WT x C*05 control (p-value $<0.01$, linear mixed effects model, Fig. 4d), also evident by a shift in radii distribution of the spiral artery vessel nodes (Fig. 4e and S3c). Furthermore, a $\sim 38 \%$ increase in the mean spiral artery segment length was observed in KIR $\mathrm{x} \mathrm{C}^{*} 05$ IUGR mice compared to WT $\mathrm{x} \mathrm{C}^{*} 05$ controls (Fig. 4f, p-value $<0.05$, linear mixed effects model), with a skew in the spiral artery segment length distributions in the opposite direction of that observed for the radii (Fig. $\mathbf{4 g}$ and S3d). There was 
a trend in the KIR $x C^{*} 05$ IUGR mice to have fewer spiral artery vessel segments ( $p=0.07$, MannWhitney test), while the total length of spiral artery vasculature and the segment volume remained unaltered (Fig. S3e-g).

Due to the skewed spiral artery length-to-diameter ratio in KIR x $C^{*} 05$ mice (Fig. 4h), we also quantified the total spiral artery network resistance by imposing a pressure gradient between the inlets and outlets and calculating the total outflow. The vascular conductance through the IUGR KIR x C*05 spiral artery network tended to be lower than in WT x $\mathrm{C}^{*} 05$ mating controls $(\mathrm{p}=0.06$, Mann-Whitney test) (Fig. 4i), suggesting a higher resistance to blood flow through the spiral artery network. Collectively, these findings demonstrated that the IUGR phenotype observed in KIR x $C^{*} 05$ matings was associated with inadequate placentation and a change in remodeling of uterine spiral arteries during gestation.

\section{Atlas of uNK cells at the mid-gestation mouse maternal-fetal interface}

To better understand uNK function and diversity at the maternal-fetal interface, we next built a single cell atlas of NK cells, using both full length scRNA-seq (with SMART-Seq2 (Picelli et al., 2014; Trombetta et al., 2014), providing deeper coverage per cell, Methods), and 3' droplet-based scRNASeq (profiling large cell numbers, Methods), profiling isolated uNK cells at gd9.5 from the three different mating groups: KIR x $\mathrm{C}^{*} 05$ (IUGR), WT x $\mathrm{C}^{*} 05$ (control 1) and $\mathrm{C}^{*} 05 / \mathrm{KIR} \times \mathrm{WT}$ mice (control 2) (Fig. 5a). For full length scRNA-seq, we first FACS sorted uNK cells (NCR1 ${ }^{+} \mathrm{CD} 3 \varepsilon^{-} \mathrm{TCRb}^{-}$ $\left.\mathrm{CD} 19^{-} \mathrm{CD} 45^{+} \mathrm{CD} 122^{+}\right)$into either trNK $\left(\mathrm{CD} 49 \mathrm{a}^{+}\right)$or $\mathrm{cNK}\left(\mathrm{DX} 5^{+}\right)$cells, collecting 1,091 and 1,085 cell profiles, respectively, across five mice each for the three mating groups (Fig. 5b, S4a-c). For droplet based scRNA-seq, we used total uNK cells, profiling 30,147 high quality uNK cells across 3-4 mice from each of the three mating groups (Fig. 5d, S4d-f). Each mouse represents cells pooled from the maternal-fetal interface of multiple implantation sites within the same litter.

Based on the full length scRNA-seq, cNK and trNK cells formed two distinct subsets in a low dimensionality embedding (Fig. 5b), and could be distinguished by signatures of differentially 
expressed genes as determined by Mann Whitney test (Fig. 5b,c). cNKs expressed cellular migration and homing markers (e.g., Ccl5, Sell), while trNKs expressed cell-cell and cell-matrix interaction markers (e.g., Adam8, Spp1). The two NK populations also had distinct granzyme profiles, with cNKs and trNKs showing higher Gzma and Gzmc expression, respectively (Fig. 5c). trNKs had more genes detected per cell (median number of genes per cell in trNKs $=4,258$ and in $\mathrm{cNKs}=2,165 ; \mathrm{p}$ value $=$ $6.96 \times 10^{-264}$, Mann Whitney U test) and a larger size by FACS (data not shown) than cNKs, consistent with higher transcriptional activity (Fig. S4a); uNK cell subset size differences have been demonstrated microscopically (Paffaro et al., 2003). Scoring the droplet-based profiles with the full-length based cNK and trNK signatures similarly distinguished the two populations in the low dimensionality embedding (Fig. 5d). As reported previously (Doisne et al., 2015), cNK cells represented a smaller proportion of total profiled uNK cells (Fig. 5d and S1n).

\section{Seven $u$ NK cell subsets in the maternal-fetal interface}

Unsupervised clustering identified six NK subsets in the full length scRNA-Seq data (Fig. 5b) and eight NK subsets in the droplet-based scRNA-Seq data (Fig. 5d); after evaluating and comparing subsets across both datasets, we annotated seven distinct NK cell subsets (concordant between the two datasets) at the maternal-fetal interface at gd9.5 (Fig. 5e, Fig. S5a,b). Due to the deeper gene coverage, we prioritised the subset-specific differentially expressed genes from full length scRNA-seq to annotate the six NK subtypes found in the full length data (Fig. 5f): (1 and 2) two cNK subtypes (cNK-1 and cNK-2) that shared several cNK marker genes, however, only cNK-2 had NF-kB inhibitors (Nfkbiz, Nfkbid, Nkfkia), (3) a trNK cell subset (trNK-1) enriched for cytokine receptor signalling genes and marked by $C d 7, C x c r 6$ and $P d c d 1$; (4) Gzmd/e/g/f-high trNKs (trNK-2) enriched for several central and protein metabolic pathways (e.g., glycolysis, $\mathrm{p}=3.1 \times 10^{-5}$, Fisher's exact test), (5) proliferating trNKs (trNK-3 or trNK-3a/3b) marked by expression of genes such as Birc5 and Ccna2, and a high gene proliferation signature score (Fig. S5c, Table S1), and (6) a subset including both cNK and trNK cells (mix-1), defined by expression of $C d 27, C d 7$ and $E m b$ (the gene signature for this mix-1 subset scores higher on cNKs than trNKs, Fig. S4g-h). Of the above six subsets, only cNK-2 did not form a distinct subset in the droplet-based data, however, scoring its signature highlights a distinguishable population 
within the cNK-1 cluster (Fig. S5a). The seventh NK subset, Gzmd/e/g/f-high trNKs expressing $P d c d 1$ (trNK-4), was a distinct subset only in the droplet-based data. Scoring its signature identified it within the trNK-2 subset in the full length data (Fig. S5b), but the cells largely overlapped cells in the full length and droplet-based trNK-2 cluster and signature, and it is possible that this subset was over-split in the droplet-based data (Fig. S5b). The eighth subset found in the droplet-based data (trNK-5) had high expression of the chemokine Ccll, but was less well defined in the droplet data (Fig. S5b) and not included as a major uNK cell subset in our final census (Fig. 5b,d,e,f).

Overall, cells from the different genotypes, KIR x C*05 (IUGR), WT x C*05 (control 1) and C*05/KIR x WT mice (control 2), had similar distributions across most of the subsets identified by clustering, suggesting that genotype did not have a dramatic effect on the overall NK cellular categories (Fig. S4in), with the exception of the Gzmd/e/g/f-high, $P d c d 1$-high trNK-4 cluster (in droplet-based scRNAseq), which was enriched for cells from the KIR $x \mathrm{C}^{*} 05$ (IUGR) genotype $(\mathrm{p}<0.05$, Dirichletmultinomial regression, as described before (Smillie et al., 2019), Fig. S4n). Subset trNK-4, however, was not distinct from trNK-2 in the full length data and shared many marker genes with trNK-2 in both datasets.

Collectively, these results highlighted the diversity of uNK cells during mid-gestation and broadly identified seven transcriptionally distinct subsets of cNK and trNK cells present at the maternal-fetal interface in control and IUGR mice.

\section{Increased lymphocyte activation and decreased ECM and tissue remodelling programs in uNK cell subsets in IUGR}

Given the limited changes in the proportion of broad uNK subsets between genotypes and the continuum of uNK cell subsets, we hypothesised that IUGR may have more nuanced changes in cell intrinsic gene programs within NK subsets. To uncover those, we used topic modelling with latent Dirichlet allocations (LDA) (Bielecki et al., 2018; Dey et al., 2017; Xu et al., 2019) to learn 16 gene programs ("topics") across the droplet based scRNA-seq profiles (Fig. S5d, Methods). This approach 
assigns each gene and cell a weight in every topic, indicating the importance of the gene to the topic and of the topic to the cell, with the top scoring genes defining the gene program for that topic. Cells and genes can be highly weighted for more than one topic.

Topics ranged from general biological processes (e.g., ribosomal biogenesis, topic 1) to regulation of NK cell function (topic 10) (Fig. S6), and included cNK/trNK specific topics and ones spanning cells from both subtypes. For example, Topic 3 ("lymphocyte migration", Fig. 5g) was enriched for genes involved in immune cell infiltration, lymphocyte migration and cytokine response and spanned trNK1, trNK-3a and mix-1 cells; Topic 8 ("transcriptional regulation and splicing" Fig. 5h) spanned cNK1, trNK-1 and mix-1 cells, and was enriched for genes involved in transcriptional regulation, spliceosome machinery and cell differentiation; and Topic 9 ("proliferation”, Fig. 5i) scored highly in the proliferating cells from both trNK (trNK-3a and trNK-3b) and cNKs (Fig. S5c). Some topics revealed gene programs that distinguish between cells within a single cluster (Fig. S6).

The cell weights for topic 6 (lymphocyte activation) and 16 (ECM and tissue remodelling) were significantly and robustly different in cells from KIR x C*05 IUGR mating vs. control matings (Fig. 6a-c, Methods). The lymphocyte activation program (Topic 6) is induced in IUGR (p-value $=5.06^{*} 10^{-}$ ${ }^{9}$, KS statistic $=-0.049$ for IUGR vs. CTR1; p-value $=4.09 * 10^{-14}, \mathrm{KS}$ statistic $=-0.073$ for IUGR vs. CTR2; KS test) (Fig. 6b), whereas the ECM and tissue remodelling program (Topic 16) is repressed $\left(\mathrm{p}\right.$-value $=3.3^{*} 10^{-3}, \mathrm{KS}$ statistic $=0.028$ for IUGR vs CTR $1 ; \mathrm{p}$-value $=3.34 * 10^{-5}, \mathrm{KS}$ statistic $=0.043$ for IUGR vs CTR2; KS test) (Fig. 6c).

The lymphocyte activation program induced in IUGR (Topic 6) is enriched for NF-kB signalling pathway, ribosomal genes, chemokine signalling and cytokine-cytokine receptor interaction genes $(\mathrm{p}=$ $0.0008,1.37 * 10^{-32}, 0.0131,0.048$, respectively; Fisher's exact test). Cell weights for topic 6 are highest in the region of the droplet-based cNK-1 subset expressing NF-kB inhibitors (aligned with full length cNK-2 subset) and in the mixed cNK/trNK subset (mix-1). In the absence of proliferation, hyperactive ribosome biogenesis can indicate cellular stress (Orsolic et al., 2016). NF-kB regulates cytokine and 
stress-induced expression of Gadd45b (a topic gene involved in regulation of cell growth and apoptosis (Jin et al., 2002; Liebermann et al., 2011)), and $C d 7$ is a topic gene involved in NK cell costimulatory triggering (Rabinowich et al., 1994). In situ hybridization on mid-gestation uterine tissue also confirmed increased expression of one of the top genes $C c l l$ in KIR x C*05 IUGR mice $v s$. controls (Fig. 6d).

The ECM and tissue remodelling program repressed in IUGR (Topic 16) has cell weights highest in the Gzmd/e/g/f-high trNK subtype (trNK-2). Top program genes (Gzmd, Gzmg, Gzme, but not Gzmf) are expressed by uNK cells in pregnancy and upregulated by IL-12 and IL-15 in uterine decidual cells (the modified uterine endometrium formed during pregnancy). These granzymes are proposed to be noncytotoxic and play a role in ECM and tissue remodelling, as well as in parturition (Allen and NilsenHamilton, 1998; Croy et al., 1997; Delgado et al., 1996). Decreased expression of Gzmd/e/g was confirmed by insitu hybridization on mid-gestation uterine tissue in KIR x C*05 IUGR mice $v$ s. controls (Fig. 6e). Other program genes (Ctsg, $M t 1$ and $M t 2)$ play roles in tissue remodelling, cell signalling and protection against oxidative damage (Gao et al., 2018; Subramanian Vignesh and Deepe, 2017). The program gene Havcr2 (or Tim-3), is associated with NK cell activation and maturation (Ndhlovu et al., 2012), and patients undergoing recurrent miscarriages have fewer Tim $3^{+} \mathrm{NK}$ cells — suggesting that these $\mathrm{Tim}^{+} \mathrm{NK}$ cells promote maternal-fetal tolerance (Li et al., 2017; Li et al., 2016; Subramanian Vignesh and Deepe, 2017).

We also tested for genes that are differentially expressed in cells from the IUGR phenotype within a specific NK cell subset (calculated using pseudobulk differential expression analysis, Methods, Fig. 6a,f). Some differentially expressed genes not highlighted by topic modeling include Fos, Acp5, Spp1, Gsto1, Map3k1, Akt3, and Il1Orb (Fig. 6f). Fos encodes for one of the proteins in the AP-1 transcription complex and plays a role in the modulation of NK cell function (Marusina et al., 2008); Acp 5 encodes an enzyme (TRAP) that regulates the activity of Osteopontin (encoded by Spp 1 - also a differentially expressed gene), which has been associated with regulation of fetal growth and recurrent spontaneous abortion (Ek-Rylander et al., 1994; Fu et al., 2017); Gstol is a stress response gene, which plays a role in redox homeostasis (Kodym et al., 1999); Akt3 is a protein kinase family member, which can also 
promote induction of reactive oxygen species (Polytarchou et al., 2020); Map3k1 integrates multiple signalling pathways and can also crosstalk with Wnt signalling (Meng et al., 2018); and Il10rb is a cell surface receptor for multiple cytokines and can stimulate activation of the JAK/STAT signaling pathway (Donnelly et al., 2004).

Together, our analyses show that in IUGR there is an increase in programs related to regulation of cell growth, apoptosis and NF-kB signalling, and decrease in programs for ECM and tissue remodelling and protection against oxidative stress.

\section{IUGR-associated transcriptional changes across diverse cell types at the maternal-fetal interface}

To understand NK cell states in the broader context of the cellular ecosystem, we next analysed 42,869 high quality droplet-based scRNA-seq profiled from all cell types at the maternal-fetal interface at midgestation ( $g d 9.5 ; n=3$ from each mating combination) and identified transcriptional differences in IUGR by cell type (Fig. 7a and S7a-c).

Unsupervised clustering and post-hoc annotation highlighted 20 cell subsets (Methods), spanning immune, stromal, decidual, trophoblast and vascular smooth muscle (vSMCs) cells (Fig. 7b), similar to findings from early human pregnancies (Vento-Tormo et al., 2018). There were nine immune cell subsets: trNKs, mixed cNKs and trNKs, (Fig. S7d), T cells, B cells, ILC2/3s, dendritic cells (Cd209a+), two macrophage subsets differing in complement ( $C l q a$ and $C 1 q b)$ and MHC II (H2-Abl and H2-Ebl) expression, and a myeloid subset (expressing Retnlg, Slpi and S100a9 (Yang et al., 2018). There were seven stromal cell subsets: one of endometrial stromal fibroblasts (Col3a1, Colla1, Colla2, Dcn, Lum, Eln and Postn) and six of cell subsets at different stages of decidualisation (critical for remodeling the uterine endometrium) as reflected by the level of Wnt signalling molecules and modulators (Wnt4, Wnt5a, Wnt6, Sfrpl) (Ramathal et al., 2010; Zhang and Yan, 2016), TGF- $\beta$ superfamily morphogen (Bmp2) (Ramathal et al., 2010; Zhang and Yan, 2016), angiogenic factors (Angpt2 and Angpt4) and steroid biosynthesis genes (Cyp 1 1a). One non-canonical subset was decidual-like, expressing Prl8a2 (a decidual prolactin-related protein that contributes to pregnancy-dependent adaptations to hypoxia 
(Bu et al., 2016)), Cyrab (important in mouse embryo implantation and decidualization) and Scgblal (encoding Uteroglobin and expressed by mucosal epithelial cells (Mukherjee et al., 2007)); these cells may support decidualisation (Fig. 7b).

There were three trophoblast subsets: Trophoblast 1 cells expressed H19, which influences trophoblast cell migration and invasion (Zuckerwise et al., 2016), and other trophoblast genes, including Bex1, Krt8 and Mest, and included cells expressing endothelial markers (e.g., Pecam1, Cd34, Tiel, Egfl7). Trophoblast 2 cells expressed $H s d 11$ b2 (an enzyme produced by trophoblast cells (Togher et al., 2014)) and the prolactin receptor Prlr, which plays a role in trophoblast invasiveness and migration (Stefanoska et al., 2013). Trophoblast 3 cells expressed keratins (Krt7, Krt8, Krt18, Prapl, expressed by the uterine luminal epithelium (Diao et al., 2010)) and the early trophoblast marker Wfdc2 (Yabe et al., 2016). Finally, VSMCs expressed actin-binding and contractile genes such as Acta2, Myl9, CnnI and Tagln (Fig. 7b).

Cell subset composition was similar across different mice and genotypes (Fig. S7e-g), but we identified IUGR-associated expression changes in cell intrinsic expression in multiple cell types, either shared across cell types, or specific to a subset (Pseudobulk differential expression analysis, Fig. 7c). For example, Anxa2 (a member of the Annexin family which plays a role in cell migration, invasion and angiogenesis (Lokman et al., 2011)) was downregulated in cells from IUGR mice compared to controls in different cell types (Fig. 7c), which we validated in situ (Fig. S7h). There were cell type-specific differentially expressed genes in many non-NK subsets. These included IUGR-up or downregulated stromal cell genes with roles in angiogenesis (Rnf213, Slit3) (Kobayashi et al., 2015; Ohkubo et al., 2015), regulation of fetal growth (Igf1, Igfbp2, Igfbp3, Igfbp4) (Nawathe et al., 2016), invasion and migration (Usp25, Prlr, Aspn, Spon2) (Ding et al., 2019; Lu et al., 2020; Satoyoshi et al., 2015; Stefanoska et al., 2013), and decidualisation and Wnt signalling (Sfrp4, Rnf213, Wnt4) (Carmon and Loose, 2008; Scholz et al., 2016). Some of these genes have also been implicated in the pathogenesis of pre-eclampsia (Sfrp4, Wnt4) (Wang et al., 2016; Zhang et al., 2013) and small-for-gestational-age pregnancies (Dcn, Igfl, Igfbp2, Igfbp3, Igfbp4) (Murthi et al., 2016; Nawathe et al., 2016) (Fig. 7c and 
S7i). Macrophages and dendritic cells showed changes in genes including Osm, involved in invasion under hypoxic conditions (Wie et al., 2018), and transcription factor E2fl that affects dendritic cell maturation (Fang et al., 2010) and is also implicated in pre-eclampsia (Kaitu'u-Lino et al., 2015). Macrophage cell subsets expressed genes were enriched for dendritic cell maturation, antigen presentation or inflammation genes $(\mathrm{FDR}=0.007,0.02,0.0001$, IPA) (Table S2). Trophoblasts and vSMCs showed changes in genes involved in remodelling of ECM (P4hal) (Gilkes et al., 2013), invasion (Klf5) (Ma et al., 2017), regulation of angiogenesis or ECM remodelling (Klf5, Cyr61, Col4a2, Bmper) (Babic et al., 1998; Heinke et al., 2008; Kamphaus et al., 2000; Shindo et al., 2002) and stress response (Sparc) (Fenouille et al., 2011) (Fig. 7c and Fig. S7j). Some of these differentially expressed genes have been associated with pre-eclampsia or IUGR (Sparc, Rgs2, Cyr61) (Gellhaus et al., 2007; Perschbacher et al., 2020; Tossetta et al., 2019; Zhang et al., 2010). This highlights how the interaction between maternal KIR2DL1 and paternal HLA-C*05 has profound effects on several placental nonNK cell types and underlines a role for these genes and cellular functions in the etiology of IUGR.

\section{Maternal KIR2DL1 and paternal HLA-C*05 lead to change in cell-cell interaction networks in \\ IUGR}

Finally, we analysed our data to identify changes in putative cell-cell interactions in IUGR vs. control mice. We identified putative interactions between cell subsets (including within the same cell type) based on expressed receptor-ligand pairs (Fig. 7a, Methods), scored significant interactions with CellPhoneDB in WT $x \mathrm{C}^{*} 05$ control and KIR $\times \mathrm{C}^{*} 05$ IUGR groups separately (Fig. 7d,e), and highlighted the interactions which were increased (red) or decreased (blue) between any pair of cell subsets in the IUGR network (Fig. 7e).

At baseline, the highest number of significant interactions occurred between stromal cells, fibroblasts, trophoblasts, and vSMCs, and both cNKs and trNKs had significant interactions with most other cell types (Fig. 7d). 38 of the 399 cell subset pairs with significant interactions in at least one condition had at least $20 \%$ more significant receptor-ligand interactions in IUGR than in WT (some as many as 50\% more). Conversely, only nine of the cell type pairs had fewer significant receptor-ligand interactions in 
IUGR (Fig. 7e). Because more cell subset pairs had an increase rather than a decrease in the number of significant interactions in IUGR, we hypothesise that there is an overall increase in predicted intercellular communication at the maternal-fetal interface in IUGR.

We next focused on putative receptor-ligand interactions between NK cells and the other cell types that were detected only in IUGR or only in controls (selected examples in Fig. 7f). For example, the putative interaction between $I l 15 r$ on NK cells and $I l 15$ on trophoblast 3 subtype was found to be significant in IUGR, but was not significant in the control group; IL-15 has been suggested to impact stromal cell decidualization and regulation of uNK cell differentiation and function (Dunn et al., 2002; Okada et al., 2000; Ye et al., 1996). Furthermore, there is a significant interaction between Spn on NK cells and Icam 1 on trophoblast subtype 1 in IUGR, but not WT cells. Indeed, overexpression of ICAM-1 by the villous trophoblast cells has been shown in placentitis and thought to play a role in the rupture of the trophoblast barrier (Juliano et al., 2006). In another example, there is a significant interaction between $T g f \beta R 1$ on NK cells and $T f g \beta 2$ on the stromal or trophoblast cell subtypes in IUGR, consistent with TGFß's control of trophoblast invasion, proliferation and migration (Davies et al., 2016). Similarly, there is a significant interaction between Notchl in NK cells and Dlk1 in trophoblast cells or SMCs in the IUGR mating but not the control group; the Notch pathway is one of the key pathways regulating angiogenesis (Zhao and Lin, 2012). Overall, these results highlight potential roles for specific putative interactions between uNK cells and other cell types that could contribute to the development of or be the result of IUGR.

\section{Discussion}

IUGR is one of the most common obstetric diseases and is associated with perinatal morbidity and mortality, as well as several long-term medical conditions such as heart disease, type 2 diabetes and stroke. While the consequences of IUGR are well understood, much is unknown about the underlying mechanisms that cause it and effective screening and treatment strategies are needed. One major challenge in studying pathogenic mechanisms of pregnancy complications is a lack of accessible tissue at relevant points early in gestation, especially when the disease manifestations are seen only later in 
pregnancy; therefore, a physiologically relevant model system for studying IGUR is necessary. Furthermore, even though there is evidence that HLA and KIR genes confer risk to IUGR, these genes are highly polymorphic and inherited in complex haplotypes in the human population (Bashirova et al., 2006; Lanier, 2005; Parham, 2005; Trowsdale, 2001; Vilches and Parham, 2002), making it challenging to dissect the role of individual genes.

Here, using a stepwise and multidisciplinary approach, we demonstrate a functional basis for how specific risk genes inherited from both parents can, in combination, lead to IUGR. Using a novel humanised transgenic model, we provide direct evidence for the involvement of the inhibitory KIR receptor, KIR2DL1, expressed on maternal uNK cells, and its interaction with fetal HLA-C*05 in manifestation of IUGR. Detailed characterisation of the model by imaging of the uteroplacental vasculature demonstrated that this genetic risk translated to changes in uterine spiral artery diameter and segment length and therefore, a higher resistance or decreased blood flow to the fetuses leading to growth restriction, implicating the KIR-HLA gene interaction in remodeling of the uterine vasculature.

IUGR and pre-eclampsia may have a shared disease pathogenesis, although mechanistic differences between these conditions have not been widely studied. In our model, we observe IUGR, evidenced by reduced average fetal weight, a fetal weight distribution skewed towards $<5^{\text {th }}$ and $10^{\text {th }}$ percentile, and placentation defects caused by altered uterine spiral arteries — but we did not see symptoms of preeclampsia (e.g. hypertension or proteinuria). It is possible that the absence of pre-eclampsia in our model is because pre-eclampsia is a disease of the human species and does not seem to occur in most other mammals (Robillard et al., 2002); however, it is also possible that manifestation of pre-eclampsia requires additional risk factors or mechanisms. Thus, our model might serve as baseline for investigating which additional factors could lead on from IUGR to pre-eclampsia.

Characterisation of cell types from the maternal-fetal interface by scRNA-seq revealed cellular heterogeneity and function as well as gene programs involved in placentation and the development of disease in pregnancy. In particular, we built a single cell atlas of sorted uNK cells from both healthy 
controls and mice with IUGR, at a time point in gestation when uNK cells are most prevalent and contribute to important biological pathways in pregnancy. We identified seven major uNK subtypes and 16 cell states. The uNK subtypes play diverse roles at the maternal-fetal interface, demonstrated by their functional heterogeneity—ranging from cellular migration, regulation of NF-kB activity, to those involved in cell-cell and cell-matrix interactions and metabolic pathways. Topic modeling revealed fluidity in cell states where cells from different uNK subtypes participated in the same gene program or a proportion of cells from within a specific subtype participated in a gene program. While the uNK cells have been shown to be important in pregnancy, our findings reveal the complex roles these cells play at a critical timepoint in pregnancy both in terms of the different biological programs and the uNK subtypes.

Both $\mathrm{cNK}$ and trNK subtypes are involved in biological processes altered or deficient in IUGR, including chemokine signaling, degradation of ECM components, and protection against oxidative damage. The involvement of uNK cells in these processes, which play an important role in placentation and vascular remodeling, demonstrated that signaling via the inhibitory receptor KIR2DL1 dampened the function or response of uNK cells, thus contributing to development of IUGR. This is concordant with previous observations that uNK cells surround spiral arteries and release angiogenic factors that play a role in placentation and vascular remodeling (Croy et al., 2000). Further work could involve studying the role of specific genes within the biological processes deficient in disease.

Profiling of unsorted cells from the mouse maternal-fetal interface at mid-gestation showed that despite the NK specific expression of KIR genes, there are substantial transcriptional changes in the IUGR model in several non-NK cell types from the maternal-fetal interface-impacting key functions such as stromal cell decidualization, Wnt signalling, angiogenesis, invasion and ECM remodeling. We hypothesize that this is a result of cellular crosstalk between uNK cells and the cells in the microenvironment, possibly though receptor-ligand interaction pairs present exclusively in the IUGR mice or control mice, between uNK cells and other cell types. 
The gene expression changes in cells at the maternal-fetal interface in IUGR were moderate in magnitude but spanned many genes and cell subsets. This combined effect is likely to be further amplified due to the sensitivity to changes to placentation and the fetus at this early and critical stage of gestation. This could imply that healthy pregnancy represents a fine tuned and highly calibrated system and a disturbance in that balance leads to a multitude of downstream effects ultimately manifesting in disease.

We saw striking similarities in genes and pathways that were differentially expressed in IUGR with those that play a role in tumour progression and metastasis. For example, processes such as cellular invasion, migration, angiogenesis, cellular proliferation and differentiation are important in placentation, but are also involved in malignancy. Unlike the uncontrollable growth that occurs in cancer, these processes, however, are normally tightly controlled in development of the placenta (Januar et al., 2015; West et al., 2018). Understanding of the mechanisms that perturb these processes in pregnancy disorders might provide insights into tumour metastasis and vice versa, and also provide a starting point for developing new drugs to intercept IUGR at the earliest possible timepoint. The safety of such drugs will clearly need to be stringent as side effects could be more severe than IUGR because of the sensitivity of the fetus.

Our data provide essential functional data to support human genetic studies associating maternal KIR AA genotype with risk for pregnancy complications when the fetal group 2 HLA-C genes are paternally inherited (Hiby et al., 2010). Specifically, we did not observe significant IUGR in the C*05/KIR x WT mating combination, where the group 2 HLA-C gene in the fetus was maternally inherited and the mother and the fetus had the same level or number of copies of group 2 HLA-C genes.

IUGR is difficult to predict and it has been found that growth restricted fetuses $>10^{\text {th }}$ percentile in size can remain undiagnosed despite not meeting their growth potential and having an increased risk for adverse perinatal outcome (Gordijn et al., 2016; Malhotra et al., 2019; Nardozza et al., 2017). Therefore, identifying risk genes and early prenatal events that lead to IUGR are crucial. Identifying specific KIR 
and HLA-C as risk genes and an understanding of how they mediate disease means that they could be used to screen parents and identify infants with increased risk of neonatal complications, thereby having the potential to allow development of new treatment modalities and improve pregnancy management and clinical outcomes for both the mother and the baby.

\section{Acknowledgments}

L.F. was supported by the Wellcome Trust (grant no. 100308/Z/12/Z), Danish National Research Foundation, Takeda, the Medical Research Council (grant no. MC_UU_12010/3), the Oak Foundation (grant no. OCAY-15-520) and the NIHR Oxford BRC. G.M. was supported by the Wellcome Trust (grant no. 100956/Z/13/Z) and the Li Ka Shing Foundation. We would like to thank V.Sexl (University of Veterinary Medicine of Vienna) for the NCR1-iCre transgenic mice, P.Höglund (Karolinska Institutet) for $\mathrm{H} 2-\mathrm{Kb} \mathrm{H} 2-\mathrm{Db}$ knockout mice, B.Davies (Wellcome Trust Centre for Human Genetics, University of Oxford) for transgenic mouse generation services, D.Bowman, A.Ortiz and S.Jerman (Indica Labs, Albuquerque, NM, USA) for RNAscope image analysis services, L. Gaffney for figure edits, G.Holländer and M.Deadman (Dept. of Paediatrics, University of Oxford) for help with TEC isolations, C.Smillie, S. Simmons, A.Haber (Broad Institute of MIT and Harvard) and A.-C.Villani (Harvard Medical School) for brainstorming and discussions, G.Douglas and V.Rashbrook (Radcliffe Dept. of Medicine, University of Oxford) for help with blood pressure measurement in mice, A. Vernet (University of Oxford) for helping with micro-CT scanning, R.Kuehn (Helmholtz Center Munich) for help with transgenic vector design. We would like to acknowledge S-A.Clark, C.Waugh, K.Clark and P.Sopp in the flow cytometry facility at the MRC WIMM for providing cell sorting services. The flow cytometry facility is supported by the MRC HIU; MRC MHU (MC_UU_12009); NIHR Oxford BRC; Kay Kendall Leukaemia Fund (KKL1057), John Fell Fund (131/030 and 101/517), the EPA fund (CF182 and CF170) and by the MRC WIMM Strategic Alliance awards G0902418 and MC_UU_12025. We also thank the MRC WIMM core Transgenic service team for providing cryopreservation services. This project has been funded in part with federal funds from the Frederick National Laboratory for Cancer Research, under Contract No. HHSN261200800001E. The content of this publication does not necessarily reflect the views or policies of the Department of Health and 
Human Services, nor does mention of trade names, commercial products, or organizations imply endorsement by the U.S. Government. This Research was supported in part by the Intramural Research Program of the NIH, Frederick National Lab, Center for Cancer Research.

\section{Author Contributions}

Conceptualization, G.K. and L.F.; Methodology, G.K., C.B.M.P., O.A., S.J.R., M.H., A.S., I.A.D. and G.M.; Software, C.B.M.P., G.K., J.L., O.A., S.J.R., M.H. and A.S.; Formal Analysis, C.B.M.P., G.K., M.A., J.L., O.A., S.J.R., M.H., A.S., K.E.A., C.D. and J.D.; Investigation, G.K., C.B.M.P., M.A., S.B.K., K.E.A., C.D., H.G.E., L.T.N., D.D., A.N., L.T.J., T.B. and E.S.; Writing - Original Draft, G.K. and C.B.M.P.; Writing - Review \& Editing, G.K., C.B.M.P., O.A., A.R. and L.F.; Funding Acquisition, L.F., A.R., O.R.-R. and G.K.; Supervision, L.F., A.R., O.R.-R., M.C.; Project Administration, G.K., C.B.M.P., O.R.-R. and L.F.

\section{Competing Interests}

A.R. is a co-founder and equity holder of Celsius Therapeutics, an equity holder in Immunitas, and was an SAB member of ThermoFisher Scientific, Syros Pharmaceuticals, Neogene Therapeutics and Asimov until July 31, 2020. From August 1, 2020, A.R. is an employee of Genentech. O.R.-R is an employee of Genentech as of October 19, 2020. O.A., O.R.-R. and A.R. are co-inventors on patent applications filed by the Broad Institute for inventions related to single cell genomics, such as in PCT/US2018/060860 and US provisional application no. 62/745,259. G.M. is a director of and shareholder in Genomics plc and a partner in Peptide Groove LLP. 


\section{Figure Legends}

Fig. 1. Development and characterisation of the HLA-C*05 and KIR2DL1 transgenic mouse model. (a) Schematic of construct used to make HLA-C*05 transgenic mice. The grey shaded region is from the $H L A-C$ allele, and the white shaded region is from the murine $H-2 K^{b}$ allele. (b and c) Representative cell surface expression of HLA-C*05 on total cells from organs (b) and on gated cell subsets from the spleen $(\mathbf{C})$. NK cells are $\mathrm{NKp} 46^{+} \mathrm{CD}^{-} \mathrm{TCR}^{-}$cells, thymus $\mathrm{TCR}+$ cells are $\mathrm{CD}^{+} \mathrm{TCR}^{+}$ and TECs are Epcam ${ }^{+} \mathrm{CD} 45^{-}$cells. Numbers denote mean florescence intensity (MFI). (d) HLA-C cell surface expression plotted as molecules of equivalent fluorochrome (MEF) (e) Representative cell surface HLA-C*05 expression on spleen $\mathrm{CD} 19^{+}$cells cultured with or without stimulation. Dashed lines represent unstimulated cells and solid lines represent stimulated cells. Numbers denote MFI. (f) HLAC cell surface expression plotted as MEF from $\mathrm{CD} 19^{+}$splenocytes (g) Schematic of KIR2DL1 and Ncr 1iCre gene expression construct used to make NK cell-specific KIR2DL1-expressing mice. (h) Representative cell surface expression of KIR2DL1 on NKp46 ${ }^{+} \mathrm{CD}^{-} \mathrm{TCR}^{-} \mathrm{NK}$ cells in different organs or on different cell subsets from spleen. Numbers denote percentage of $\mathrm{KIR}^{+}$cells. (i) Percentage of $\mathrm{KIR}^{+}$cells in immune cell subsets from the spleen. (j and $\mathbf{k}$ ) Cell surface staining of CD27 and CD11b on gated splenic NK cells. HLA-C*05 mice are shown in purple, WT mice in grey, KIR2DL1expressing mice in pink and HLA-C*05/KIR2DL1 double transgenic mice in green. Mean + SEM is shown. (d) $n=3-10$ per group. (f) $n=4-7$ per group. (i) $n=4-11$ per group. (k) $n=4-10$ per group. *p $<0.05,{ }^{* *} \mathrm{p}<0.01,{ }^{* * *} \mathrm{p}<0.001,{ }^{* * * *} \mathrm{p}<0.0001$, Mann-Whitney test. 
Fig. 2. The KIR2DL1 transgene recognises HLA-C*05 and modifies NK cell effector function, and transgene expression is verified at the maternal-fetal interface. (a) Experimental design of NK cell stimulation experiments. (b) Staining for IFN $\gamma^{+} \mathrm{NK}$ cells $\left(\mathrm{CD}^{-} \mathrm{TCR}^{-} \mathrm{Nkp}^{-} 6^{+}\right.$or $\mathrm{CD}^{-} \mathrm{TCR}^{-} \mathrm{DX} 5^{+}$) upon culture with antibodies depicted in panel (a). (b) Experimental design of adoptive transfer experiments. (d) Representative staining of cellular injection mix and $\mathrm{CFSE}^{+}$splenocytes harvested from recipient mice. Numbers denote percentage of each gated cell population. (e) Relative survival of HLA-C $* 05^{+} \mathrm{KO}$ cells compared to survival of HLA-C*05-KO cells (normalised to WT mice). (f) Representative flow cytometric staining of DX5 and CD49a on uNK cells (CD3-CD19-TCR ${ }^{-}$ $\mathrm{CD} 45^{+} \mathrm{NKp} 46^{+} \mathrm{CD} 122^{+}$cells) isolated from the implantation sites at gd9.5 from the different mating crosses. KIR staining is shown on total uNK cells, CD49a ${ }^{+}$or DX5 $5^{+}$uNK subsets. (g) Percentage of $\mathrm{KIR}^{+} \mathrm{uNK}$ cells in different mating crosses at gd9.5. Crosses are mentioned as female x male. (h) HLA$C^{*} 05$ mRNA expression on fetal trophoblast cells from implantation sites at gd9.5 or gd10.5. (i) Representative HLA-C*05 cell surface expression on fetal trophoblast cells isolated from placenta at gd12.5, and cultured with or without stimulation. Dashed lines represent unstimulated cells and solid lines represent stimulated cells. HLA-C*05 ${ }^{+}$trophoblast cells are shown in purple and HLA-C*05trophoblast i.e. WT cells are shown in grey. The filled histogram represents fluorescence minus one (FMO) control. Numbers denote MFI. (j) HLA-C cell surface expression plotted as MEF normalised to FMO controls. Mean + SEM is shown. (b) $n=4-9$ per group for $\alpha$-Ly49D stimulation, $n=3-10$ per group for $\alpha$-NKp46 stimulation. (e) $n=6-9$ per group. (g) $n=8-10$ per group. $(\mathbf{h}) n=4$ per group. (j) $n$ $=5$ per group. ${ }^{*} \mathrm{p}<0.05,{ }^{* *} \mathrm{p}<0.01, * * * \mathrm{p}<0.001, * * * * \mathrm{p}<0.0001$, Mann-Whitney test. 
Fig. 3. Expression of maternal KIR2DL1 and paternal HLA-C*05 leads to intrauterine growth restriction in fetuses. (a) Fetal weight and (c) placental weight determined at gd18.5 of progeny from different mating combinations involving WT, KIR2DL1-expressing and HLA-C*05 transgenic mice. Crosses are mentioned as female $\mathrm{x}$ male. Mean \pm SEM is shown. $\mathrm{n}=8-12$ litters per group. ${ }^{*} \mathrm{p}<0.05$, $* * \mathrm{p}<0.01, * * * \mathrm{p}<0.001$, linear mixed effects model. (b) Table showing distribution of fetal weight proportions from each mating combination compared to the WT x $C^{*} 05$ control mating. Numbers depict percentages of fetuses whose weight was below the $5^{\text {th }}$ percentile, between $5^{\text {th }}-10^{\text {th }}$ percentile and above the $10^{\text {th }}$ percentile of the WT $x \mathrm{C}^{*} 05$ mating controls. 
Fig. 4. Combination of maternal KIR2DL1 and paternal HLA-C*05 leads to changes in uterine spiral arteries during gestation. (a) Representative uteroplacental circulation from $\mathrm{WT}$ x $\mathrm{C}^{*} 05$ mating at gd10.5 visualised following perfusion with an X-ray contrast agent and imaging by micro-CT. Spiral arteries are shown in dark purple and the yolk sac is shown in yellow. (b and c) Representative spiral artery vasculature images from the different mating crosses at gd10.5. (d and f) Spiral artery diameter (d) or segment length (f) is shown. (e and g) Radii distribution of spiral artery vessel nodes (e) and distribution of segment lengths (g) is shown. (h) Ratio of the mean of the spiral artery segment length and mean of the spiral artery diameter of each implantation site is shown. (i) Total network fluid conductance based on the Poiseuille flow model. Mean \pm SEM is shown. (d, e, f, g and h) $n=17-21$ implantation sites per group. (i) $\mathrm{n}=8-10$ implantation sites per group. ${ }^{*} \mathrm{p}<0.05$, **p $<0.01, * * * \mathrm{p}<$ 0.001. Linear mixed effects model used for (d) and (f). Mann-Whitney test used for (h) and (i). 
Fig. 5. Heterogeneity of NK cells at the mouse maternal-fetal interface. (a) Overview of study design. (b) UMAP embedding of full length scRNA-seq on sorted NK cells colored by subtype as cNK or trNK (determined by FACS sorting and verified using the cNK and trNK signature score) or by Louvain cluster label. (c) Dot plot showing top six cNK and trNK marker genes. (d) UMAP embedding of NK cells profiled by droplet-based scRNA-seq and colored by Louvain cluster or the cNK and trNK signature score. (e) Summary of NK subtypes identified in sorted uNK cells derived from both full length and droplet based scRNA-seq data. (F) Dot plot of top five marker genes for each cluster shown in panel (b). (g-i) Left: top twenty genes driving the topic; the 'score' has been scaled to improve visualization. Right: UMAP embedding of droplet based scRNA-seq uNK data (same as in panel d) colored by the weight of each cell in the topic. Topic $3(\mathrm{G})$, Topic $8(\mathrm{H})$, Topic 9 (I). $\mathrm{n}=2176$ cells for full length and 30,147 cells for droplet-based NK data. In all plots, cells from all three genotypes (WT x $C^{*} 05$, KIR $x C^{*} 05, C^{*} 05 / K I R \times$ WT) are included. In dot plots, the size of the dot represents the fraction of cells with nonzero expression of each gene, and the color of the dot represents the average nonzero gene expression. 
Fig. 6. Characterisation of the IUGR phenotype in NK cells by topic modeling and differentially expressed genes (a) Overview of IUGR phenotype characterisation by topic modeling and differential gene expression analysis. (b-c) Topic modeling of droplet-based scRNA-seq uNK cell data from the mouse maternal-fetal interface. The cell topic weight distributions for the displayed topics (Topic 6 (b), and Topic 16 (c) were significantly different between KIR x C*05 vs. control mating groups. Left: top 20 genes driving the topic; the 'score' has been scaled to improve visualization. Right, top: UMAP embedding colored by the cell weight for that topic - bright red indicates the cell is high in the topic and dark blue indicates the cell is low in the topic. (d, e) Re-validation of $C c l l$ (d) and Gzme/d/g (e) from the topics using in-situ hybridisation on implantation sites at gd9.5. Representative probe staining on sections from the different mating crosses is shown. Quantification depicted as percentage of positive cells or average probe copies per cell for the respective probes within a stained section is shown. (f) Volcano plots showing differentially expressed genes in the IUGR KIR x $C^{*} 05$ mating combination. Differentially expressed genes were calculated on a per cluster basis, and are stratified as such in the panel. (d, e) n=13-14 sections for $C c l 1$ and n=15-16 sections for Gzmd/e/g, Mann-Whitney test. 
Fig. 7. Characterisation of cell types, the IUGR phenotype and cell-cell interactions in unsorted cells from the maternal-fetal interface. (a) Overview of scRNA-seq analysis of unsorted cells. (b) UMAP embedding of unsorted cells from all three genotypes analysed using droplet-based scRNA-seq and colored by cell type classification. (c) Heatmap of select differentially expressed genes between KIR x C*05 and WT x C*05 mating groups. Differentially expressed genes were calculated on a per cell type basis, and every gene in this heatmap was differentially expressed in at least one cell type. The color of each square indicates the log of the average expression fold change between KIR $x C^{*} 05$ and WT x $C^{*} 05$. Bright red - higher average expression in KIR $\times \mathrm{C}^{*} 05$; Dark blue - lower average expression in KIR x C*05; White - no difference; grey - the gene was expressed in less than $10 \%$ in both conditions within a cell subset. The colored boxes to the left of the heatmap indicate the top functional annotation of each gene. (d-e) Cell-cell interaction analysis by CellPhoneDB. Cell-cell interaction network for WT x $C^{*} 05$ cells $(\mathbf{d})$ and KIR $\times C^{*} 05$ cells (e). The color of each circle corresponds to the cell type classification on the UMAP in panel (b), and the weight of each line represents the number of significant ligand-receptor interactions found between the cell type nodes on either end, with a thicker line indicating a great number of significant interactions. Lines are only shown between cell type nodes if at least 50 significant interactions were found. Interactions increased or decreased in the KIR $\times C^{*} 05$ group compared to the WT $\times C^{*} 05$ group are shown in red and blue, respectively. (f) Select ligand-receptor pairs that were found to be unique in either KIR x C*05 or WT $\mathrm{x} \mathrm{C}^{*} 05$ groups in one of the NK cell types. The first gene listed in the label was found in one of the NK clusters, and the second gene was found in an interacting non-NK cell type cluster. A large circle indicates that the receptor-ligand pair was found between an NK cluster and the indicated cell type; cell types are colored as in panel (b). For example, $A p p_{-} C d 74$ was found between one of the NK subtypes and macrophages 1 , macrophages 2 and dendritic cells in the KIR x C*05 genotype, but not in the WT x $\mathrm{C}^{*} 05$ genotype. 


\section{Supplementary Figure Legends}

\section{Fig. S1. Functional characterisation of the HLA-C*05 and KIR2DL1 transgenic mouse model. (a)} HLA-C*05 mRNA expression using tissue from different organs from WT or HLA-C*05 transgenic mice. Data is shown normalised to a HLA-C*05 spleen sample. (b) Representative HLA-C*05 cell surface expression on total spleen cells cultured with or without stimulation. Dashed lines represent unstimulated cells and solid lines represent stimulated cells. HLA-C*05 and WT mice are shown in purple and grey respectively. Numbers denote MFI. (c) HLA-C cell surface expression plotted as MEF of total splenocytes cultured with or without stimulation. (d) Percentage of NK cells (CD3-TCR ${ }^{-}$ $\mathrm{NKp} 46^{+}$cells) in spleen, $\mathrm{LN}$ and bone marrow $(\mathrm{BM})$ from the different transgenic mice. (e and f) Percentage of total $\mathrm{CD}^{+} \mathrm{CD}^{+}, \mathrm{CD}^{+} \mathrm{CD}^{+}$and $\mathrm{CD} 19^{+}$cells in the spleen and $\mathrm{LN}$ in the different transgenic mice. (g) Percentage of splenic NK cells expressing the different NK cell surface receptors/maturation markers as highlighted in the plot (percentages of receptor expression is gated on $\mathrm{CD}^{-} \mathrm{TCR}^{-} \mathrm{NKp} 46^{+} \mathrm{NK}$ cells). (h) Percentage of splenic NK cells expressing transcription factors T-bet and Eomes in the different transgenic mice. (i) Proportion of Ly49 ${ }^{+} \mathrm{NK}$ cells (stained using antibody clones 14B11 and 4D11) in the LN and BM in the different transgenic mice. (j and $\mathbf{k}$ ) Cell surface staining of CD27 and CD11b on gated NK cells from the bone marrow $(\mathbf{j})$ or $\mathrm{LN}(\mathbf{k})$ from the different transgenic mice. (l) Response of KIR2DL1-expressing YT NK cell line (measured by IFN- $\gamma$ production by flow cytometry) when co-cultured with 721.221 cells alone or 721.221 cells transduced with lentiviral HLA-C*05, HLA-C*07 or vector-transduced cells or with stimulation with PMA and Calcium Ionomycin. Additional controls included co-culture with 721.221 cells that were transduced with HLAC*05 and HLA-C*07 constructs where the HLA $\alpha 3$ domain, transmembrane and cytoplasmic regions were cloned in from the respective HLA-C alleles (see Methods for details). (m) Percentage of uterine NK cells $\left(\mathrm{CD} 3{ }^{-} \mathrm{CD} 19^{-} \mathrm{TCR}^{-} \mathrm{CD} 45^{+} \mathrm{NKp} 46^{+} \mathrm{CD} 122^{+}\right)$at the maternal-fetal interface at $\mathrm{gd} 9.5$ are shown from the different transgenic crosses. Mating crosses are mentioned as female x male. (n) Percentage of $\mathrm{CD}_{49 \mathrm{a}^{+}}$or $\mathrm{DX}^{+}$uNK cell subsets at gd9.5 from the different transgenic crosses. Crosses are mentioned as female $x$ male. Mean \pm SEM is shown. (a) $n=3-9$ per group. (c) $n=4-7$ per group. (d) $n$ 
= 3-11 per group. (e) $n=4-16$ per group. (f) $n=3-10$ per group. $(\mathbf{g}) n=3-12$ per group. $(\mathbf{h}) n=3-10$ per group. (i) $n=3-9$ per group. (j and $\mathbf{k}) \mathrm{n}=3-10$ per group. (l) $n=8-9$ per condition $(\mathbf{m}$ and $\mathbf{n}) \mathrm{n}=$ 8-10 per group. ${ }^{*} \mathrm{p}<0.05,{ }^{* *} \mathrm{p}<0.01,{ }^{* * *} \mathrm{p}<0.001,{ }^{* * * *} \mathrm{p}<0.0001$, Mann-Whitney test. 
Fig. S2. Assessment of blood pressure, urinary protein and plasma proteins. (a) Systolic blood pressure measured using non-invasive tail-cuff photoplethysmography during pregnancy and postpartum in the depicted transgenic mating crosses. Grey line indicates birth of the pups. Blood pressure at each time point is shown relative to the baseline for each mouse (measured pre-pregnancy). (b) Assessment of protein in urine samples collected from gd18.5 pregnant mice from the different transgenic mating crosses. Urinary albumin/creatinine is shown. (c, d and e) Plasma concentrations of sFlt-1 (c), PIGF-2 (d), sEndoglin (e) in pregnant gd18.5 mice from the different transgenic mating crosses. (a) $\mathrm{n}=7-9$ per group. Not all mice gave valid blood pressure measurements on every measurement day. Represented data includes observations from a minimum of 3 mice per group on any measured day. (b) $n=8-10$ per group. (c) $n=7-10$ per group. $(\mathbf{d}$ and $\mathbf{e}) \mathrm{n}=6-8$ per group. Mean \pm SEM is shown. Mann-Whitney test. 
Fig. S3. Changes in uterine spiral arteries during gestation. (a and b) Representative skeletonised vascular network from the segmentation analysis from the WT $\mathrm{x} \mathrm{C}^{*} 05$ and $\mathrm{KIR} \times \mathrm{C}^{*} 05$ mating crosses at gd10.5 are shown. (c and d) Cumulative distributions of vessel radii nodes (c) and segment lengths (d) from the mating crosses are depicted. (e) The number of spiral artery vessel segments are shown. (f and g) Total length of vasculature (f) and volume (g) measured from the spiral artery segmented data from WT x C*05 and KIR x C*05 mating crosses is shown. Data is represented as Mean \pm SEM. (c g) $\mathrm{n}=17-21$ implantation sites per group. Mann-Whitney test. 


\section{Fig. S4. Quality control metrics for full length and droplet-based scRNA-seq data of sorted NK}

cells at the mouse maternal-fetal interface. (a) Distribution of the number of counts per cell and the number of genes per cell in each genotype for the full length sorted NK data. The first and third quartiles and median are indicated on each violin plot. The genotypes are displayed along the $\mathrm{x}$ axis, and conventional NK cells are indicated by “_cNK” and tissue resident NK cells are indicated by "_trNK". (b-c) UMAP embedding of sorted NK cells from the maternal fetal interface, analyzed by full length scRNA-Seq. Colors of the dots correspond to genotype (b) or the mouse of origin (c). (d) Distribution of the number of UMI per cell, the number of genes per cell in each genotype from the droplet based scRNA-seq uNK data. The first and third quartiles and median are indicated on each violin plot. (e-f) UMAP embedding of sorted NK cells analyzed by droplet based scRNA-seq, with cells colored by genotype (e) or mouse of origin (f). (g) Cells expressing the gene signature from the mix-1 cell subset from the full length scRNA-seq data stratified into cNK or trNK cells based on FACS sorting. (h) Proportion of cNK and trNK cells (defined by FACS) in each cell subset for the full length scRNA-seq NK dataset. (i-k) Proportion of composition of clusters depicted in Fig. 5B as per individual mice (i) or genotype (j, k). (l-n) Proportion of composition of clusters depicted in Fig. 5D as per individual mice (l) or genotype $(\mathbf{m}, \mathbf{n})$. p-values represent whether the number of cells belonging to the IUGR phenotype (KIR x C*05 mating) are significantly more or less than the two control phenotypes, and were calculated using a latent Dirichlet allocation model. 
Fig. S5. Comparison of NK subtype signatures observed between the full length and droplet based scRNA-seq datasets. (a) NK subtype (cluster) signatures from full length scRNA-Seq data, plotted on UMAP embeddings for both full length and droplet based scRNA-seq data sets to highlight consistency in observed heterogeneity between the two data sets. Red indicates a cell has a high signature score and blue indicates the cell has a low signature score. (b) NK subtype (cluster) signatures from the droplet based scRNA-seq data set, plotted on UMAP embeddings for both full length and droplet based scRNAseq data sets (the reverse of b). (c) UMAP embedding of droplet based scRNA-seq NK data, colored by proliferation score. (d) Akaike information criterion (AIC) and Bayesian information criterion (BIC) for iterations of topic modeling where $\mathrm{k}$ was set to $4: 20$ in increments of 2 ; this information was used to help select the value of $\mathrm{k}$ to use for our final topic modeling analysis. 
bioRxiv preprint doi: https://doi.org/10.1101/2021.03.26.437292; this version posted March 28, 2021. The copyright holder for this preprint

(which was not certified by peer review) is the author/funder. All rights reserved. No reuse allowed without permission.

Fig. S6. Topic modeling of droplet based scRNA-seq NK cell data from the maternal-fetal

interface. Topics from topic modeling analysis depicting all 16 topics. UMAP embedding colored by the cell weight for that topic - bright red indicates the cell is high in the topic and dark blue indicates the cell is low in the topic. 
Fig. S7. Quality control metrics for droplet based scRNA-seq data of unsorted cells at the mouse maternal-fetal interface and differentially expressed pathways. (a) Distribution of the number of UMI per cell and the number of genes per cell in each genotype, for droplet based scRNA-seq data from unsorted cells. (b-c) UMAP embedding of unsorted cells analysed by droplet based scRNA-seq colored by genotype (b) or mouse of origin (c). (d) UMAP embedding of droplet based scRNA-seq data from unsorted cells colored by cNK and trNK signature score. (e) Proportion of composition of clusters/cell types depicted in Fig. 7b stratified by individual mice (e) or genotype (f,g). (h) Re-validation of Anxa2 using in-situ hybridisation (RNAscope) on uterine tissue collected from implantation sites at gd9.5. Representative probe staining on sections from WT x C*05 or KIR x C*05 implantation sites is shown. Anxa2 staining is seen in teal. Quantification of probe staining depicted as average probe copies per cell within a stained section is shown. $\mathrm{n}=10$ sections, Mann-Whitney test. (i,j) Comparison of pathways and functions altered in stromal cell types (i), Trophoblast and SMCs (j) in KIR x C*05 IUGR mice compared to the controls, assessed using Ingenuity pathway analysis. Colors of heatmap indicate $\mathrm{BH}-$ FDR value of enrichment. 


\section{Supplementary Tables}

Table S1 - Signatures used in QC

Table S2 - Pathway analysis using IPA on differentially expressed genes on selected cell type clusters from droplet-based unsorted cells data

Table S3 - List of oligonucleotides

\section{References}

Adamson, S.L., Lu, Y., Whiteley, K.J., Holmyard, D., Hemberger, M., Pfarrer, C., and Cross, J.C. (2002). Interactions between trophoblast cells and the maternal and fetal circulation in the mouse placenta. Dev Biol 250, 358-373.

Allen, M.P., and Nilsen-Hamilton, M. (1998). Granzymes D, E, F, and G are regulated through pregnancy and by IL-2 and IL-15 in granulated metrial gland cells. J Immunol 161, 2772-2779.

Apps, R., Murphy, S.P., Fernando, R., Gardner, L., Ahad, T., and Moffett, A. (2009). Human leucocyte antigen (HLA) expression of primary trophoblast cells and placental cell lines, determined using single antigen beads to characterize allotype specificities of anti-HLA antibodies. Immunology 127, 26-39.

Ashkar, A.A., Black, G.P., Wei, Q., He, H., Liang, L., Head, J.R., and Croy, B.A. (2003). Assessment of requirements for IL-15 and IFN regulatory factors in uterine NK cell differentiation and function during pregnancy. J Immunol 171, 2937-2944.

Ashkar, A.A., Di Santo, J.P., and Croy, B.A. (2000). Interferon gamma contributes to initiation of uterine vascular modification, decidual integrity, and uterine natural killer cell maturation during normal murine pregnancy. J Exp Med 192, 259-270.

Bashirova, A.A., Martin, M.P., McVicar, D.W., and Carrington, M. (2006). The killer immunoglobulin-like receptor gene cluster: tuning the genome for defense. Annu Rev Genomics Hum Genet 7, 277-300.

Bernstein, I.M., Horbar, J.D., Badger, G.J., Ohlsson, A., and Golan, A. (2000). Morbidity and mortality among very-low-birth-weight neonates with intrauterine growth restriction. The Vermont Oxford Network. Am J Obstet Gynecol 182, 198-206.

Bielecki, P., Riesenfeld, S.J., Kowalczyk, M.S., Amezcua Vesely, M.C., Kroehling, L., Yaghoubi, P., Dionne, D., Jarret, A., Steach, H.R., McGee, H.M., et al. (2018). Skin inflammation driven by differentiation of quiescent tissue-resident ILCs into a spectrum of pathogenic effectors. bioRxiv.

Borenstein, S.H., Graham, J., Zhang, X.L., and Chamberlain, J.W. (2000). CD8+ T cells are necessary for recognition of allelic, but not locus-mismatched or xeno-, HLA class I transplantation antigens. J Immunol 165, 2341-2353.

Borg, A.J., Yong, H.E., Lappas, M., Degrelle, S.A., Keogh, R.J., Da Silva-Costa, F., Fournier, T., Abumaree, M., Keelan, J.A., Kalionis, B., et al. (2015). Decreased STAT3 in human idiopathic fetal growth restriction contributes to trophoblast dysfunction.

Reproduction 149, 523-532. 
Brady, J., Hayakawa, Y., Smyth, M.J., and Nutt, S.L. (2004). IL-21 induces the functional maturation of murine NK cells. J Immunol 172, 2048-2058.

Brosens, I., Pijnenborg, R., Vercruysse, L., and Romero, R. (2011). The "Great Obstetrical Syndromes" are associated with disorders of deep placentation. Am J Obstet Gynecol 204, 193-201.

Bu, P., Alam, S.M., Dhakal, P., Vivian, J.L., and Soares, M.J. (2016). A Prolactin Family Paralog Regulates Placental Adaptations to a Physiological Stressor. Biol Reprod 94, 107. Burton, G.J., and Jauniaux, E. (2018). Pathophysiology of placental-derived fetal growth restriction. Am J Obstet Gynecol 218, S745-S761.

Chiossone, L., Chaix, J., Fuseri, N., Roth, C., Vivier, E., and Walzer, T. (2009). Maturation of mouse NK cells is a 4-stage developmental program. Blood 113, 5488-5496.

Conde-Agudelo, A., Papageorghiou, A.T., Kennedy, S.H., and Villar, J. (2013). Novel biomarkers for predicting intrauterine growth restriction: a systematic review and metaanalysis. BJOG 120, 681-694.

Croy, B.A., Ashkar, A.A., Minhas, K., and Greenwood, J.D. (2000). Can Murine Uterine Natural Killer Cells Give Insights Into the Pathogenesis of Preeclampsia? Journal of the Society for Gynecologic Investigation 7, 12-20.

Croy, B.A., McBey, B.A., Villeneuve, L.A., Kusakabe, K., Kiso, Y., and van den Heuvel, M. (1997). Characterization of the cells that migrate from metrial glands of the pregnant mouse uterus during explant culture. J Reprod Immunol 32, 241-263.

de Onis, M., Blossner, M., and Villar, J. (1998). Levels and patterns of intrauterine growth retardation in developing countries. Eur J Clin Nutr 52 Suppl 1, S5-15.

Delgado, S.R., McBey, B.A., Yamashiro, S., Fujita, J., Kiso, Y., and Croy, B.A. (1996). Accounting for the peripartum loss of granulated metrial gland cells, a natural killer cell population, from the pregnant mouse uterus. J Leukoc Biol 59, 262-269.

Diao, H., Xiao, S., Zhao, F., and Ye, X. (2010). Uterine luminal epithelium-specific prolinerich acidic protein 1 (PRAP1) as a marker for successful embryo implantation. Fertil Steril 94, 2808-2811 e2801.

Doisne, J.M., Balmas, E., Boulenouar, S., Gaynor, L.M., Kieckbusch, J., Gardner, L., Hawkes, D.A., Barbara, C.F., Sharkey, A.M., Brady, H.J., et al. (2015). Composition, Development, and Function of Uterine Innate Lymphoid Cells. J Immunol 195, 3937-3945. Du, M.R., Wang, S.C., and Li, D.J. (2014). The integrative roles of chemokines at the maternal-fetal interface in early pregnancy. Cell Mol Immunol 11, 438-448.

Duhig, K.E., Myers, J., Seed, P.T., Sparkes, J., Lowe, J., Hunter, R.M., Shennan, A.H., Chappell, L.C., and group, P.t. (2019). Placental growth factor testing to assess women with suspected pre-eclampsia: a multicentre, pragmatic, stepped-wedge cluster-randomised controlled trial. Lancet 393, 1807-1818.

Dunn, C.L., Critchley, H.O., and Kelly, R.W. (2002). IL-15 regulation in human endometrial stromal cells. J Clin Endocrinol Metab 87, 1898-1901.

Eckelhart, E., Warsch, W., Zebedin, E., Simma, O., Stoiber, D., Kolbe, T., Rulicke, T., Mueller, M., Casanova, E., and Sexl, V. (2011). A novel Ncr1-Cre mouse reveals the essential role of STAT5 for NK-cell survival and development. Blood 117, 1565-1573. Ego, A., Zeitlin, J., Batailler, P., Cornec, S., Fondeur, A., Baran-Marszak, M., Jouk, P.S., Debillon, T., and Cans, C. (2013). Stillbirth classification in population-based data and role of fetal growth restriction: the example of RECODE. BMC Pregnancy Childbirth 13, 182. Figueras, F., and Gardosi, J. (2011). Intrauterine growth restriction: new concepts in antenatal surveillance, diagnosis, and management. Am J Obstet Gynecol 204, 288-300.

Forsen, T., Eriksson, J., Tuomilehto, J., Reunanen, A., Osmond, C., and Barker, D. (2000). The fetal and childhood growth of persons who develop type 2 diabetes. Ann Intern Med 133, 176-182. 
Frankel, S., Elwood, P., Sweetnam, P., Yarnell, J., and Smith, G.D. (1996). Birthweight, body-mass index in middle age, and incident coronary heart disease. Lancet 348, 1478-1480. Fu, B., Zhou, Y., Ni, X., Tong, X., Xu, X., Dong, Z., Sun, R., Tian, Z., and Wei, H. (2017). Natural Killer Cells Promote Fetal Development through the Secretion of Growth-Promoting Factors. Immunity 47, 1100-1113 e1106.

Gao, S., Zhu, H., Zuo, X., and Luo, H. (2018). Cathepsin G and Its Role in Inflammation and Autoimmune Diseases. Arch Rheumatol 33, 498-504.

Gardosi, J., Kady, S.M., McGeown, P., Francis, A., and Tonks, A. (2005). Classification of stillbirth by relevant condition at death (ReCoDe): population based cohort study. BMJ 331, 1113-1117.

Gordijn, S.J., Beune, I.M., Thilaganathan, B., Papageorghiou, A., Baschat, A.A., Baker, P.N., Silver, R.M., Wynia, K., and Ganzevoort, W. (2016). Consensus definition of fetal growth restriction: a Delphi procedure. Ultrasound Obstet Gynecol 48, 333-339.

Gotthardt, D., and Sexl, V. (2016). STATs in NK-Cells: The Good, the Bad, and the Ugly. Front Immunol 7, 694.

Griffith, J.W., Sokol, C.L., and Luster, A.D. (2014). Chemokines and chemokine receptors: positioning cells for host defense and immunity. Annu Rev Immunol 32, 659-702.

Hanna, J., Goldman-Wohl, D., Hamani, Y., Avraham, I., Greenfield, C., Natanson-Yaron, S., Prus, D., Cohen-Daniel, L., Arnon, T.I., Manaster, I., et al. (2006). Decidual NK cells regulate key developmental processes at the human fetal-maternal interface. Nat Med 12, 1065-1074.

Hiby, S.E., Apps, R., Chazara, O., Farrell, L.E., Magnus, P., Trogstad, L., Gjessing, H.K., Carrington, M., and Moffett, A. (2014). Maternal KIR in combination with paternal HLA-C2 regulate human birth weight. J Immunol 192, 5069-5073.

Hiby, S.E., Apps, R., Sharkey, A.M., Farrell, L.E., Gardner, L., Mulder, A., Claas, F.H., Walker, J.J., Redman, C.W., Morgan, L., et al. (2010). Maternal activating KIRs protect against human reproductive failure mediated by fetal HLA-C2. J Clin Invest 120, 4102-4110. Hiby, S.E., Walker, J.J., O'Shaughnessy K, M., Redman, C.W., Carrington, M., Trowsdale, J., and Moffett, A. (2004). Combinations of maternal KIR and fetal HLA-C genes influence the risk of preeclampsia and reproductive success. J Exp Med 200, 957-965.

Horikoshi, M., Beaumont, R.N., Day, F.R., Warrington, N.M., Kooijman, M.N., FernandezTajes, J., Feenstra, B., van Zuydam, N.R., Gaulton, K.J., Grarup, N., et al. (2016). Genomewide associations for birth weight and correlations with adult disease. Nature 538, 248-252. Huang, R.P., Ozawa, M., Kadomatsu, K., and Muramatsu, T. (1993). Embigin, a member of the immunoglobulin superfamily expressed in embryonic cells, enhances cell-substratum adhesion. Dev Biol 155, 307-314.

Imdad, A., and Bhutta, Z.A. (2013). Nutritional management of the low birth weight/preterm infant in community settings: a perspective from the developing world. J Pediatr 162, S107114.

J, E.D., Pollheimer, J., Yong, H.E., Kokkinos, M.I., Kalionis, B., Knofler, M., and Murthi, P. (2016). Epithelial-mesenchymal transition during extravillous trophoblast differentiation. Cell Adh Migr 10, 310-321.

Januar, V., Desoye, G., Novakovic, B., Cvitic, S., and Saffery, R. (2015). Epigenetic regulation of human placental function and pregnancy outcome: considerations for causal inference. Am J Obstet Gynecol 213, S182-196.

Jha, P., and Das, H. (2017). KLF2 in Regulation of NF-kappaB-Mediated Immune Cell Function and Inflammation. Int J Mol Sci 18.

Jin, R., De Smaele, E., Zazzeroni, F., Nguyen, D.U., Papa, S., Jones, J., Cox, C., Gelinas, C., and Franzoso, G. (2002). Regulation of the gadd45beta promoter by NF-kappaB. DNA Cell Biol 21, 491-503. 
Juliano, P.B., Blotta, M.H., and Altemani, A.M. (2006). ICAM-1 is overexpressed by villous trophoblasts in placentitis. Placenta 27, 750-757.

Karre, K., Ljunggren, H.G., Piontek, G., and Kiessling, R. (1986). Selective rejection of H-2deficient lymphoma variants suggests alternative immune defence strategy. Nature 319, 675678.

King, A., Burrows, T.D., Hiby, S.E., Bowen, J.M., Joseph, S., Verma, S., Lim, P.B., Gardner, L., Le Bouteiller, P., Ziegler, A., et al. (2000). Surface expression of HLA-C antigen by human extravillous trophoblast. Placenta 21, 376-387.

Krolak-Olejnik, B., Beck, B., and Olejnik, I. (2006). Umbilical serum concentrations of chemokines (RANTES and MGSA/GRO-alpha) in preterm and term neonates. Pediatr Int 48, 586-590.

Lanier, L.L. (2005). NK cell recognition. Annu Rev Immunol 23, 225-274.

Leavenworth, J.W., Verbinnen, B., Wang, Q., Shen, E., and Cantor, H. (2015). Intracellular osteopontin regulates homeostasis and function of natural killer cells. Proc Natl Acad Sci U S A 112, 494-499.

Leon, D.A., Lithell, H.O., Vagero, D., Koupilova, I., Mohsen, R., Berglund, L., Lithell, U.B., and McKeigue, P.M. (1998). Reduced fetal growth rate and increased risk of death from ischaemic heart disease: cohort study of 15000 Swedish men and women born 1915-29. BMJ 317, 241-245.

Leskinen, M.J., Lindstedt, K.A., Wang, Y., and Kovanen, P.T. (2003). Mast cell chymase induces smooth muscle cell apoptosis by a mechanism involving fibronectin degradation and disruption of focal adhesions. Arterioscler Thromb Vasc Biol 23, 238-243.

Levine, R.J., Maynard, S.E., Qian, C., Lim, K.H., England, L.J., Yu, K.F., Schisterman, E.F., Thadhani, R., Sachs, B.P., Epstein, F.H., et al. (2004). Circulating angiogenic factors and the risk of preeclampsia. N Engl J Med 350, 672-683.

Li, Y., Lorca, R.A., and Su, E.J. (2018). Molecular and cellular underpinnings of normal and abnormal human placental blood flows. J Mol Endocrinol 60, R9-R22.

Li, Y., Zhang, J., Zhang, D., Hong, X., Tao, Y., Wang, S., Xu, Y., Piao, H., Yin, W., Yu, M., et al. (2017). Tim-3 signaling in peripheral NK cells promotes maternal-fetal immune

tolerance and alleviates pregnancy loss. Sci Signal 10.

Li, Y.H., Zhou, W.H., Tao, Y., Wang, S.C., Jiang, Y.L., Zhang, D., Piao, H.L., Fu, Q., Li, D.J., and Du, M.R. (2016). The Galectin-9/Tim-3 pathway is involved in the regulation of

NK cell function at the maternal-fetal interface in early pregnancy. Cell Mol Immunol 13, 7381.

Liebermann, D.A., Tront, J.S., Sha, X., Mukherjee, K., Mohamed-Hadley, A., and Hoffman, B. (2011). Gadd45 stress sensors in malignancy and leukemia. Crit Rev Oncog 16, 129-140. Lima, P.D., Croy, B.A., Degaki, K.Y., Tayade, C., and Yamada, A.T. (2012). Heterogeneity in composition of mouse uterine natural killer cell granules. J Leukoc Biol 92, 195-204.

Lima, P.D., Zhang, J., Dunk, C., Lye, S.J., and Croy, B.A. (2014). Leukocyte driven-decidual angiogenesis in early pregnancy. Cell Mol Immunol 11, 522-537.

Lokman, N.A., Ween, M.P., Oehler, M.K., and Ricciardelli, C. (2011). The role of annexin A2 in tumorigenesis and cancer progression. Cancer Microenviron 4, 199-208.

Maghazachi, A.A. (2010). Role of Chemokines in the Biology of Natural Killer Cells. In The Chemokine System in Experimental and Clinical Hematology, O. Bruserud, ed. (Berlin, Heidelberg: Springer Berlin Heidelberg), pp. 37-58.

Malhotra, A., Allison, B.J., Castillo-Melendez, M., Jenkin, G., Polglase, G.R., and Miller, S.L. (2019). Neonatal Morbidities of Fetal Growth Restriction: Pathophysiology and Impact. Front Endocrinol (Lausanne) 10, 55. 
McCowan, L.M., Figueras, F., and Anderson, N.H. (2018). Evidence-based national guidelines for the management of suspected fetal growth restriction: comparison, consensus, and controversy. Am J Obstet Gynecol 218, S855-S868.

Meyer, N., Woidacki, K., Knofler, M., Meinhardt, G., Nowak, D., Velicky, P., Pollheimer, J., and Zenclussen, A.C. (2017). Chymase-producing cells of the innate immune system are required for decidual vascular remodeling and fetal growth. Sci Rep 7, 45106.

Moffett, A., and Loke, C. (2006). Immunology of placentation in eutherian mammals. Nat Rev Immunol 6, 584-594.

Moraitis, A.A., Wood, A.M., Fleming, M., and Smith, G.C. (2014). Birth weight percentile and the risk of term perinatal death. Obstet Gynecol 124, 274-283.

Mukherjee, A.B., Zhang, Z., and Chilton, B.S. (2007). Uteroglobin: a steroid-inducible immunomodulatory protein that founded the Secretoglobin superfamily. Endocr Rev 28, $707-$ 725 .

Nakimuli, A., Chazara, O., Hiby, S.E., Farrell, L., Tukwasibwe, S., Jayaraman, J., Traherne, J.A., Trowsdale, J., Colucci, F., Lougee, E., et al. (2015). A KIR B centromeric region present in Africans but not Europeans protects pregnant women from pre-eclampsia. Proc Natl Acad Sci U S A 112, 845-850.

Nardozza, L.M., Caetano, A.C., Zamarian, A.C., Mazzola, J.B., Silva, C.P., Marcal, V.M., Lobo, T.F., Peixoto, A.B., and Araujo Junior, E. (2017). Fetal growth restriction: current knowledge. Arch Gynecol Obstet 295, 1061-1077.

Ndhlovu, L.C., Lopez-Verges, S., Barbour, J.D., Jones, R.B., Jha, A.R., Long, B.R., Schoeffler, E.C., Fujita, T., Nixon, D.F., and Lanier, L.L. (2012). Tim-3 marks human natural killer cell maturation and suppresses cell-mediated cytotoxicity. Blood 119, 3734-3743.

Okada, S., Okada, H., Sanezumi, M., Nakajima, T., Yasuda, K., and Kanzaki, H. (2000). Expression of interleukin-15 in human endometrium and decidua. Mol Hum Reprod 6, 75-80. Orsolic, I., Jurada, D., Pullen, N., Oren, M., Eliopoulos, A.G., and Volarevic, S. (2016). The relationship between the nucleolus and cancer: Current evidence and emerging paradigms. Semin Cancer Biol 37-38, 36-50.

Otsuka, A., Hanafusa, T., Kono, N., and Tarui, S. (1991). Lipopolysaccharide augments HLA-A,B,C molecule expression but inhibits interferon-gamma-induced HLA-DR molecule expression on cultured human endothelial cells. Immunology 73, 428-432.

Paffaro, V.A., Jr., Bizinotto, M.C., Joazeiro, P.P., and Yamada, A.T. (2003). Subset classification of mouse uterine natural killer cells by DBA lectin reactivity. Placenta 24, 479488.

Parham, P. (2005). MHC class I molecules and KIRs in human history, health and survival. Nat Rev Immunol 5, 201-214.

Parrish-Novak, J., Dillon, S.R., Nelson, A., Hammond, A., Sprecher, C., Gross, J.A., Johnston, J., Madden, K., Xu, W., West, J., et al. (2000). Interleukin 21 and its receptor are involved in NK cell expansion and regulation of lymphocyte function. Nature 408, 57-63.

Rabacal, W., Pabbisetty, S.K., Hoek, K.L., Cendron, D., Guo, Y., Maseda, D., and Sebzda, E. (2016). Transcription factor KLF2 regulates homeostatic NK cell proliferation and survival. Proc Natl Acad Sci U S A 113, 5370-5375.

Rabinowich, H., Pricop, L., Herberman, R.B., and Whiteside, T.L. (1994). Expression and function of CD7 molecule on human natural killer cells. J Immunol 152, 517-526.

Ramathal, C.Y., Bagchi, I.C., Taylor, R.N., and Bagchi, M.K. (2010). Endometrial decidualization: of mice and men. Semin Reprod Med 28, 17-26.

Resnik, R. (2002). Intrauterine growth restriction. Obstet Gynecol 99, 490-496. Rich-Edwards, J.W., Stampfer, M.J., Manson, J.E., Rosner, B., Hankinson, S.E., Colditz, G.A., Willett, W.C., and Hennekens, C.H. (1997). Birth weight and risk of cardiovascular disease in a cohort of women followed up since 1976. BMJ 315, 396-400. 
Riva, A., and Chokshi, S. (2018). Immune checkpoint receptors: homeostatic regulators of immunity. Hepatol Int 12, 223-236.

Robillard, P.Y., Dekker, G.A., and Hulsey, T.C. (2002). Evolutionary adaptations to preeclampsia/eclampsia in humans: low fecundability rate, loss of oestrus, prohibitions of incest and systematic polyandry. Am J Reprod Immunol 47, 104-111.

Romo, A., Carceller, R., and Tobajas, J. (2009). Intrauterine growth retardation (IUGR): epidemiology and etiology. Pediatr Endocrinol Rev 6 Suppl 3, 332-336.

Rosenberg, A. (2008). The IUGR newborn. Semin Perinatol 32, 219-224.

Sato, Y., Higuchi, T., Yoshioka, S., Tatsumi, K., Fujiwara, H., and Fujii, S. (2003).

Trophoblasts acquire a chemokine receptor, CCR1, as they differentiate towards invasive phenotype. Development 130, 5519-5532.

Schroder, K., and Tschopp, J. (2010). The inflammasomes. Cell 140, 821-832.

Sharpe, A.H., and Pauken, K.E. (2018). The diverse functions of the PD1 inhibitory pathway. Nat Rev Immunol 18, 153-167.

Sojka, D.K., Plougastel-Douglas, B., Yang, L., Pak-Wittel, M.A., Artyomov, M.N., Ivanova, Y., Zhong, C., Chase, J.M., Rothman, P.B., Yu, J., et al. (2014). Tissue-resident natural killer (NK) cells are cell lineages distinct from thymic and conventional splenic NK cells. Elife 3, e01659.

Sojka, D.K., Yang, L., Plougastel-Douglas, B., Higuchi, D.A., Croy, B.A., and Yokoyama, W.M. (2018). Cutting Edge: Local Proliferation of Uterine Tissue-Resident NK Cells during Decidualization in Mice. J Immunol 201, 2551-2556.

Steegers, E.A., von Dadelszen, P., Duvekot, J.J., and Pijnenborg, R. (2010). Pre-eclampsia. Lancet 376, 631-644.

Stefanoska, I., Jovanovic Krivokuca, M., Vasilijic, S., Cujic, D., and Vicovac, L. (2013). Prolactin stimulates cell migration and invasion by human trophoblast in vitro. Placenta 34, 775-783.

Subramanian Vignesh, K., and Deepe, G.S., Jr. (2017). Metallothioneins: Emerging Modulators in Immunity and Infection. Int J Mol Sci 18.

Tantyo, N.A., Karyadi, A.S., Rasman, S.Z., Salim, M.R.G., Devina, A., and Sumarpo, A. (2019). The prognostic value of S100A10 expression in cancer. Oncol Lett 17, 1417-1424. Tchougounova, E., Lundequist, A., Fajardo, I., Winberg, J.O., Abrink, M., and Pejler, G. (2005). A key role for mast cell chymase in the activation of pro-matrix metalloprotease- 9 and pro-matrix metalloprotease-2. J Biol Chem 280, 9291-9296.

Togher, K.L., Togher, K.L., O'Keeffe, M.M., O'Keeffe, M.M., Khashan, A.S., Khashan, A.S., Gutierrez, H., Gutierrez, H., Kenny, L.C., Kenny, L.C., et al. (2014). Epigenetic regulation of the placental HSD11B2 barrier and its role as a critical regulator of fetal development. Epigenetics 9, 816-822.

Trowsdale, J. (2001). Genetic and functional relationships between MHC and NK receptor genes. Immunity 15, 363-374.

Tyrrell, J.S., Yaghootkar, H., Freathy, R.M., Hattersley, A.T., and Frayling, T.M. (2013). Parental diabetes and birthweight in 236030 individuals in the UK biobank study. Int $\mathbf{J}$ Epidemiol 42, 1714-1723.

Vento-Tormo, R., Efremova, M., Botting, R.A., Turco, M.Y., Vento-Tormo, M., Meyer, K.B., Park, J.E., Stephenson, E., Polanski, K., Goncalves, A., et al. (2018). Single-cell reconstruction of the early maternal-fetal interface in humans. Nature 563, 347-353. Vilches, C., and Parham, P. (2002). KIR: diverse, rapidly evolving receptors of innate and adaptive immunity. Annu Rev Immunol 20, 217-251.

Warrington, N.M., Beaumont, R.N., Horikoshi, M., Day, F.R., Helgeland, O., Laurin, C., Bacelis, J., Peng, S., Hao, K., Feenstra, B., et al. (2019). Maternal and fetal genetic effects on birth weight and their relevance to cardio-metabolic risk factors. Nat Genet 51, 804-814. 
West, R.C., Bouma, G.J., and Winger, Q.A. (2018). Shifting perspectives from "oncogenic" to oncofetal proteins; how these factors drive placental development. Reprod Biol Endocrinol $16,101$.

Xu, H., Ding, J., Porter, C.B.M., Wallrapp, A., Tabaka, M., Ma, S., Fu, S., Guo, X., Riesenfeld, S.J., Su, C., et al. (2019). Transcriptional Atlas of Intestinal Immune Cells Reveals that Neuropeptide alpha-CGRP Modulates Group 2 Innate Lymphoid Cell Responses. Immunity 51, 696-708 e699.

Yabe, S., Alexenko, A.P., Amita, M., Yang, Y., Schust, D.J., Sadovsky, Y., Ezashi, T., and Roberts, R.M. (2016). Comparison of syncytiotrophoblast generated from human embryonic stem cells and from term placentas. Proc Natl Acad Sci U S A 113, E2598-2607.

Yang, J., Anholts, J., Kolbe, U., Stegehuis-Kamp, J.A., Claas, F.H.J., and Eikmans, M. (2018). Calcium-Binding Proteins S100A8 and S100A9: Investigation of Their Immune Regulatory Effect in Myeloid Cells. Int J Mol Sci 19.

Ye, W., Zheng, L.M., Young, J.D., and Liu, C.C. (1996). The involvement of interleukin (IL)-15 in regulating the differentiation of granulated metrial gland cells in mouse pregnant uterus. J Exp Med 184, 2405-2410.

Zeisler, H., Llurba, E., Chantraine, F., Vatish, M., Staff, A.C., Sennstrom, M., Olovsson, M., Brennecke, S.P., Stepan, H., Allegranza, D., et al. (2016). Predictive Value of the sFlt-1:P1GF Ratio in Women with Suspected Preeclampsia. N Engl J Med 374, 13-22.

Zhang, Q., and Yan, J. (2016). Update of Wnt signaling in implantation and decidualization. Reprod Med Biol 15, 95-105.

Zhao, W.X., and Lin, J.H. (2012). Notch signaling pathway and human placenta. Int J Med Sci 9, 447-452.

Zuckerwise, L., Li, J., Lu, L., Men, Y., Geng, T., Buhimschi, C.S., Buhimschi, I.A., Bukowski, R., Guller, S., Paidas, M., et al. (2016). H19 long noncoding RNA alters trophoblast cell migration and invasion by regulating TbetaR3 in placentae with fetal growth restriction. Oncotarget 7, 38398-38407. 


\section{METHODS}

\section{Animals}

All animal experiments were approved by the local Ethical Review Committee at the University of Oxford, and performed under license from the UK home office (project license numbers 30/3386 and P0A53015F) in accordance with the Animals (Scientific Procedures) Act, 1986. Mice were maintained in a pathogen-free facility in individually ventilated cages in a temperature- and humidity-controlled room with a $12 \mathrm{~h}$ light $/ 12 \mathrm{~h}$ dark cycle under standard housing conditions with continuous access to food and water. Male and female mice were used across different experiments. None of the mice had any noticeable health abnormalities. For timed-mating experiments, male and female mice were mated in the afternoon of one day and checked for plugs and separated on the morning of the next day. Detection of the plug was considered as gd0.5. In addition, female mice were weighed prior to being set-up in timed-mating experiments, and on every alternate day from gd7.5 onwards in order to confirm pregnancy. These refinements avoided wastage of any pregnant transgenic mice.

\section{Generation of transgenic mice}

The HLA-C*05 and KIR2DL1 transgenic mice were generated using targeted insertion into the ROSA26 locus. For generation of the HLA-C*05 transgenic mice, to facilitate interaction of HLA-C with murine CD8 (and hence the T cell receptor), the $\alpha 3$ domain of HLA-C*05 was replaced with its murine counterpart from $\mathrm{H} 2-\mathrm{K}^{\mathrm{b}}$, along with the adjacent transmembrane and cytoplasmic domains. To allow for this, the $H L A-C^{*} 05$ construct was made by amplifying $\sim 2.04 \mathrm{~kb}$ genomic fragment of $H L A$ $C^{*} 05: 01: 01: 01$, which contained 776 bp of the $H L A-C 5^{\prime}$ UTR and exons 1-3 up to a midpoint in intron 3 , which was fused to $\mathrm{a} \sim 3.58 \mathrm{~kb}$ fragment of the genomic $H-2 K^{b}$ gene, beginning at a midpoint in intron 3 and containing exons $4-8$ and the $H-2 K^{b} 3^{\prime}$ UTR. The genomic DNA construct was first assembled in the pTZ18U vector (Sigma-Aldrich) prior to subcloning into vector pCB92 (Chen et al., 2011), downstream of a promoterless neomycin selection cassette and a murine H19 insulator, creating plasmid CB92- $\mathrm{C}^{*} 05$ in which the whole transgene array is flanked by PhiC31 attB sites. 
For generation of the KIR2DL1 transgenic mice, the coding DNA sequence of KIR2DL1*0030201 along with a Kozak consensus sequence (GCCACC) immediately upstream of the ATG start codon, was amplified and cloned into the pEx-CAG-stop-bpA vector (a gift from Ralf Kühn), between the loxP flanked STOP cassette and the polyadenylation (pA) signal. The presence of the STOP cassette (a puromycin resistance coding region followed by triple pA signals) served as the transcriptional STOP signal for transgene expression. The KIR2DL1 protein is expressed from the CAG promoter upon the Cre mediated excision of the loxP flanked stop cassette. This vector also included the promoterless neomycin selection cassette and PhiC31 attB sites required for PhiC31 integrase mediated cassette exchange.

For targeted insertion at the Gt(ROSA26)Sor locus, a PhiC31 integrase mediated cassette exchange approach was adopted using IDG26.10-3 ES cells, which are a (C57BL/6J x 129S6/SvEvTac) F1 ES cell line harbouring a PGK promoter driven hygromycin selection cassette flanked by PhiC31 attP sites, positioned within intron 1 of Gt(ROSA26)Sor (Hitz et al., 2007). The targeted insertion of the vector into the ROSA26 locus ensures reproducible transgene expression. $1 \times 10^{6}$ IDG26.10-3 ES cells (Hitz et al., 2007) were co-electroporated with $5 \mu \mathrm{g}$ of either pCB92-C*05 or pEx-CAG-KIR2DL1 and $5 \mu \mathrm{g}$ of pPhiC31o (Addgene) using the Neon transfection system (Thermo Fisher) $(3 \times 1400 \mathrm{~V}, 10 \mathrm{~ms})$ and plated on G418 resistant fibroblast feeder layers. After approximately 7 days of selection in $350 \mu \mathrm{g} / \mathrm{ml}$ G418, 16 resistant colonies were isolated per construct, expanded and screened for the correct cassette exchange event at the $5^{\prime}$ and $3^{\prime}$ ends using specific screening primers provided in Table S3 (5' screen: yields a 280 bp product; 3' screen: yields a 518 bp product). Correctly integrated ES cell clones were injected into mouse $\mathrm{C} 57 \mathrm{BL} / 6 \mathrm{~J}$ blastocysts, and the resulting chimeric males were mated to $\mathrm{C} 57 \mathrm{BL} / 6 \mathrm{~J}$ females and the progeny were screened for germline transgene transmission, normal breeding and appropriate expression of the transgene. The mice were further backcrossed onto C57BL/6J for 4-5 generations. Mice at the same backcrossing generation were used as transgene-negative littermates or controls within each experiment. The NK1.1 antigen is not expressed on the 129 background. The NK1.1 antigen status was always assessed when performing matings and the NK1.1 expression was kept consistent within experiments with mice largely being maintained on a NK1.1-ve background. 
KIR2DL1-floxed transgenic mice were mated to NCR1-iCre mice (a gift from Veronika Sexl) (Eckelhart et al., 2011) to obtain KIR2DL1-NCR1-iCre transgenic mice which had specific expression of KIR2DL1 in NCR1-expressing cells, and referred to as KIR2DL1-expressing mice hereafter. KIR2DL1-NCR1-iCre transgenic mice were crossed to HLA-C*05 transgenic mice to generate double transgenic mice having expression of both HLA-C*05 and KIR2DL1.

For adoptive transfer experiments, HLA-C*05 mice were mated to $\mathrm{H} 2-\mathrm{K}^{\mathrm{b}} \mathrm{H} 2-\mathrm{D}^{\mathrm{b}}$ double-knockout mice (a gift of Petter Höglund) to generate HLA-C*05 mice which lacked expression of $\mathrm{H} 2-\mathrm{K}^{\mathrm{b}}$ and $\mathrm{H} 2-\mathrm{D}^{\mathrm{b}}$. Genotyping of all alleles was done using PCR as described below.

\section{Cell lines}

The B-lymphoblastoid cell line, 721.221 was purchased from the International Histocompatibility Working Group and grown in RPMI-1640 supplemented with 15\% heat-inactivated fetal calf serum (FCS) (Sigma-Aldrich), sodium pyruvate (Thermo Fisher), L-glutamine (Sigma-Aldrich) and primocin (Invivogen). YT cells transfected with KIR2DL1 (a gift of Dr. Chiwen Chang) were grown in RPMI1640 supplemented with 10\% heat-inactivated FCS, L-glutamine and penicillin-streptomycin (SigmaAldrich). HEK 293T cells (ATCC) were cultured in DMEM supplemented with 10\% heat-inactivated FCS, L-glutamine and penicillin-streptomycin.

\section{Genotyping of mice}

Genomic DNA from mice and embryos was extracted using E-Z $96{ }^{\circledR}$ Tissue DNA Kit (Omega Bio-tek) as per the manufacturer's instructions. The expression level of genes of interest for each sample was normalized against mouse housekeeping gene, Glyceraldehyde 3-phosphate Dehydrogenase (GAPDH). Genotyping of HLA-C*05, KIR2DL1, NCR1 and NK1.1 was performed using real-time quantitative PCR (qPCR) on a LightCycler $480 \mathrm{II}$ (Roche) under the following conditions: $50^{\circ} \mathrm{C}$ for $2 \mathrm{~min}, 95^{\circ} \mathrm{C}$ for $10 \mathrm{~min}, 40 \times$ Quantification mode $\left(95^{\circ} \mathrm{C}\right.$ for $15 \mathrm{~s}, 60^{\circ} \mathrm{C}$ for $\left.1 \mathrm{~min}\right)$, Melting Curve mode $\left(95^{\circ} \mathrm{C}\right.$ for 15 $\mathrm{s}, 60^{\circ} \mathrm{C}$ for $15 \mathrm{~s}, 95^{\circ} \mathrm{C}$ Continuous Acquisition of 5 per $\left.{ }^{\circ} \mathrm{C}\right), 40^{\circ} \mathrm{C}$ for 10 min. qPCR samples were prepared with Power SYBR Green Master Mix (Thermo Fisher) and gene-specific primers as listed in Table S3. Comparative CT method $\left(2^{-\Delta \Delta \mathrm{CT}}\right)$ was used for the quantitative analysis of relative gene 
expression, where $\triangle \mathrm{CT}$ is the difference between the threshold cycle of gene of interest and GAPDH, and $\Delta \Delta \mathrm{CT}$ is the difference between the $\Delta \mathrm{Ct}$ of each sample and the positive control sample. Genotyping of $\mathrm{H} 2-\mathrm{K}^{\mathrm{b}} \mathrm{H} 2-\mathrm{D}^{\mathrm{b}}$ double-knockout mice was performed using Touchdown (TD) PCR on a SimpliAmp Thermal Cycler (Thermo Fisher) under the following conditions: $95^{\circ} \mathrm{C}$ for $10 \mathrm{~min}, 17$ cycles of $\left(95^{\circ} \mathrm{C}\right.$ for $30 \mathrm{~s}, 60^{\circ} \mathrm{C}$ decreased by $0.5^{\circ} \mathrm{C}$ every cycle for $40 \mathrm{~s}, 72^{\circ} \mathrm{C}$ for $\left.30 \mathrm{~s}\right), 16$ cycles of $\left(95^{\circ} \mathrm{C}\right.$ for $30 \mathrm{~s}, 60^{\circ} \mathrm{C}$ for $40 \mathrm{~s}, 72^{\circ} \mathrm{C}$ for $\left.30 \mathrm{~s}\right), 72^{\circ} \mathrm{C}$ for $10 \mathrm{~min}$. TD-PCR samples were prepared with AmpliTaq Gold ${ }^{\mathrm{TM}}$ DNA Polymerase, Gold Buffer, MgCl2, GeneAmp ${ }^{\mathrm{TM}}$ dNTP Blend (Thermo Fisher) and gene-specific primers as listed in Table S3. TD-PCR products were electrophoresed in $1.2 \%$ TrisBorate-EDTA (TBE) agarose gels, stained with Midori Green Advance DNA Stain (Geneflow) and imaged with Gel Doc XR+ Gel Documentation System (Bio-Rad).

\section{Preparation of single cell suspensions from mouse organs for phenotypic and functional characterisation experiments}

All organs were placed in R10 medium (details below) on ice until further processing. Single cell suspensions were counted using an improved Neubauer chamber or Countess I (ThermoFisher) after dilution in $0.4 \%$ trypan blue (ThermoFisher). All centrifugation steps were done at $480 \mathrm{~g}$ for $5 \mathrm{~min}$ at $4^{\circ} \mathrm{C}$ unless otherwise stated. R10 constituted of RPMI-1640 media supplemented with L-glutamine, sodium bicarbonate (Sigma-Aldrich), 10\% heat-inactivated FCS, 1x Penicillin-Streptomycin and 50 $\mu \mathrm{M}$ 2-Mercaptoethanol (Gibco), freshly added. Spleens were carefully dissociated using the plunger of a $5 \mathrm{ml}$ syringe and filtered through pre-wetted $70 \mu \mathrm{m}$ cell strainers (Corning). Cells were washed once in R10 and red blood cells lysed in Red Blood Cell Lysing Buffer Hybri-Max ${ }^{\mathrm{TM}}$ (Sigma-Aldrich) for 7 min at room temperature. Cells were washed in R10 and counted. Splenocytes were either used for cell surface and intranuclear flow cytometric staining or processed for in vitro stimulations. In some experiments, cells were also resuspended in RLT buffer (Qiagen) supplemented with 2Mercaptoethanol and stored at $-80^{\circ} \mathrm{C}$ until RNA isolation. For isolation of cells from lymph nodes, left and right inguinal and axillary lymph nodes were carefully dissociated using the plunger of a $5 \mathrm{ml}$ syringe and filtered through pre-wetted $70 \mu \mathrm{m}$ cell strainers. Cells were washed in R10, counted and 
then stained as per the flow cytometry protocol. Bone marrow isolations were done by collecting left and right tibia and flushing bones with 5ml Hanks Balanced Salt solution (HBSS) (Sigma-Aldrich) using a syringe with a $26 \mathrm{G}$ needle. Cells were dissociated by passing the bone marrow through the needle 5-10 times and filtered through pre-wetted $70 \mu \mathrm{m}$ cell strainers. Cells were washed once in R10 and red blood cells lysed in Red Blood Cell Lysing Buffer Hybri-Max ${ }^{\mathrm{TM}}$ for 7 min at RT. Cells were washed in R10, counted and then stained as per the flow cytometry staining protocol. Isolation of thymocytes were performed by dissociating thymi using the plunger of a $5 \mathrm{ml}$ syringe and filtered through pre-wetted $70 \mu \mathrm{m}$ cell strainers. Cells were washed in PBS, counted and then stained as per the flow cytometry staining protocol.

\section{Flow cytometric staining}

Surface staining was done in the presence of anti-CD16/32 antibodies to block Fc $\gamma$ RII/III receptors using Mouse Fc block ${ }^{\mathrm{TM}}$ (BD Biosciences). Cells were incubated with the Mouse $\mathrm{Fc}$ block $^{\mathrm{TM}}$ for 10 min followed by incubation with fluorochrome-conjugated pre-titrated antibodies for $20 \mathrm{~min}$ at $4^{\circ} \mathrm{C}$. Cells were washed in FACS buffer (PBS with 2\% Heat-inactivated FCS) and fixed in 1x BD cellFIX (BD Biosciences). Samples were acquired on the LSRFortessa (BD Biosciences), Fortessa X-20 (BD Biosciences), Cyan ADP (Dako) or the Attune acoustic focusing cytometer (Applied Biosystems), and analysed using the FlowJo software (FlowJo). In order to ensure comparability, FluoroSpheres (Dako) and Sphero ${ }^{\mathrm{TM}}$ Rainbow Calibration beads (BD Biosciences) both with defined MEF (molecules of equivalent fluorochromes) were run for each experiment in addition to the samples and used to calculate normalised MFI values. For intracellular staining, cells were fixed, permeabilised and stained using the BD Cytofix/Cytoperm kit (BD biosciences) as per manufacturer's instructions. For nuclear staining of transcription factors, cells were stained using the Foxp3 staining buffer set (eBioscience) according to the manufacturer's instructions. Samples were acquired and analysed as described above.

\section{Stimulation of splenocytes}


For in vitro stimulation of total splenocytes, $2 \times 10^{6}$ splenocytes were stimulated with $4 \mu \mathrm{g} / \mathrm{ml}$ LPS (Sigma-Aldrich) or 50U/ml IFN $\gamma$ (Peprotech) for 24 hours in 24-well plates in R10 medium. After incubation, cells were detached by careful pipetting, washed in FACS buffer and stained as per the flow cytometric staining protocol. For in vitro stimulation of NK cells, $1 \times 10^{6}$ splenocytes were isolated from mice and cultured on Immulon U-bottom plates coated with antibodies for Ly49D $(0.5 \mathrm{mg} / \mathrm{ml})$ (Biolegend) or NKp46 (1mg/ml) (Thermo Fisher) with or without the presence of anti-KIR antibody $(1 \mathrm{mg} / \mathrm{ml})$ (Biolegend), in the presence of $2000 \mathrm{U} / \mathrm{ml} \mathrm{rhIL-2} \mathrm{(Peprotech)} \mathrm{and} \mathrm{Brefeldin} \mathrm{A}(10 \mu \mathrm{g} / \mathrm{ml})$ (Sigma-Aldrich) for 5 hours. Cells were then harvested and stained as per the flow cytometric staining protocol and IFN $\gamma$ production by NK cells was assessed. Negative and positive controls included cells cultured without any stimulus and those cultured with PMA (250ng/ml) (Sigma-Aldrich) and Calcium Ionomycin $(2.5 \mu \mathrm{g} / \mathrm{ml})$ (Sigma-Aldrich).

\section{Adoptive transfer experiments}

Adoptive transfer experiments to assess in vivo rejection were performed as previously described (Oberg et al., 2004). Splenocytes were isolated from mice lacking expression of murine MHC class I i.e. $\mathrm{H} 2 \mathrm{~K}^{\mathrm{b}-\mathrm{H}} 2 \mathrm{D}^{\mathrm{b}-}$ (referred to as $\mathrm{KO}$ cells), WT mice (which were $\mathrm{H} 2 \mathrm{~K}^{\mathrm{b}+} \mathrm{H} 2 \mathrm{D}^{\mathrm{b}+}$ ) or HLA-C*05 expressing mice which had been bred to mice lacking expression of murine MHC class I molecules (i.e. HLA-C $* 05^{+} \mathrm{H} 2 \mathrm{~K}^{\mathrm{b}-} \mathrm{H} 2 \mathrm{D}^{\mathrm{b}-}$, referred to as HLA-C $* 05^{+} \mathrm{KO}$ cells). Cells were labelled with differential concentrations of CFSE to allow for cellular discrimination as follows: KO cells ( $0.5 \mu \mathrm{M}$ of CFSE), WT cells $\left(5 \mu \mathrm{M}\right.$ CFSE) and HLA-C $* 05^{+} \mathrm{KO}\left(5 \mu \mathrm{M}\right.$ CFSE). Cells were mixed and $\sim 5 \times 10^{6}$ cells per population were injected intravenously into either WT or KIR2DL1-expressing recipient mice. Splenocytes were isolated from recipient mice after 20 hours and cells were analysed by flow cytometry using antibodies for HLA-C (B1.23.2) and $\mathrm{H} 2 \mathrm{~K}^{\mathrm{b}}(48-5958-82)$. A small sample of the injection mix was kept and analysed by flow cytometry for reference. Survival of both HLA-C* $05^{+} \mathrm{KO}$ and KO cells was calculated relative to the WT cells in each experiment using the injection mix as reference. Relative survival of HLA-C* $05^{+} \mathrm{KO}$ compared to survival of KO cells was plotted.

\section{Isolation of thymic epithelial cells (TECs)}


TECs were isolated as previously described (Jain and Gray, 2014). In brief, thymus lobes were collected from 6-7 week old mice, were cleaned from fat and connective tissue and the capsule was removed under a dissection microscope. Cleaned tissue was digested in PBS, 0.4 Wunsch Units $/ \mathrm{ml} \mathrm{Liberase} \mathrm{TM}^{\mathrm{TM}}$ (Sigma-Aldrich) and $300 \mu \mathrm{g} / \mathrm{ml}$ DNaseI (VWR) pre-warmed to $37^{\circ} \mathrm{C}$. Tissue was carefully triturated in a step-wise manner. First, after 5 min incubation in $37^{\circ} \mathrm{C}$ water bath, the thymus lobes were carefully pipetted up and down 10-15 times using a cut P1000 pipette tip. Tissue fragments were left to settle down for $5 \mathrm{~min}$, the cell suspension transferred into a collection tube containing R10 and cells pelleted. Cells were re-suspended in FACS buffer containing $50 \mu \mathrm{g} / \mathrm{ml}$ DNaseI to prevent cell clumping. Trituration of the remaining tissue fragments was repeated a total of four rounds; with pipette tip openings gradually getting smaller and the last round using an uncut P10 tip. Cells were pooled and counted. An aliquot was taken for flow cytometric staining as a pre-TEC isolation sample. $\mathrm{CD}^{+} 5^{+}$cells were depleted using CD45 MicroBeads (Miltenyi Biotech) according to manufacturer's instructions. Cells were filtered sequentially through $70 \mu \mathrm{m}$ and $40 \mu \mathrm{m}$ (Corning) cell strainers with $1.8 \mathrm{mg} / \mathrm{ml}$ DNaseI added in order to prevent blocking of the MACS columns. The $\mathrm{CD}^{-} 5^{-}$cell fraction eluted from the LS columns (Miltenyi Biotech) represented TECs. Cells were counted and flow cytometric surface marker staining was performed as described above.

\section{Lentiviral transduction and in vitro co-culture assays}

The HLA-C*05 DNA construct (used to make transgenic mice) and a similarly designed HLA-C*07 control DNA construct were sub-cloned into the lentiviral pHRsinUbEm expression plasmid (a gift from J.M. Boname/ P.J. Lehner, University of Cambridge) as described previously (Kaur et al., 2017). To confirm that the presence of the $\mathrm{H} 2-\mathrm{K}^{\mathrm{b}} \alpha 3$ domain in the HLA-C construct did not hamper recognition of the HLA-C molecule by KIR2DL1, two additional constructs, $\mathrm{C}^{*} 05(\alpha 3-\mathrm{H})$ and $\mathrm{C}^{*} 07$ $(\alpha 3-\mathrm{H})$ constructs, were made. In the $\mathrm{C}^{*} 05(\alpha 3-\mathrm{H})$ and $\mathrm{C}^{*} 07(\alpha 3-\mathrm{H})$ DNA constructs, the $\alpha 3$ domain and hence the exons 4-8 of the HLA-C molecule were taken from the respective HLA-C genes. The lentiviral HLA-C expression plasmids were co-transfected with the vesicular stomatitis virus-G envelope plasmid pMD2.G (Addgene) and packaging plasmid psPAX2 (Addgene), containing HIV-1 
Gag and Rev, into HEK 293T cells to package lentiviral particles. Viral titres were determined by serial dilution and transduction of HEK 293T cells. 721.221 cells were transduced with the packaged lentivirus at a multiplicity of infection of 20, in the presence of polybrene (Santa Cruz Biotechnology), added at a final concentration of $8 \mu \mathrm{g} / \mathrm{ml}$. Cells were harvested 72 hours post transduction and HLA-C expression determined by staining with anti-HLA (W6/32) (Biolegend) antibody. The HLA-C transduced 721.221 cells were then co-cultured with YT-KIR2DL1 cells at a ratio of 0.5:1 T:E for 6 hours and IFN $\gamma$ production by the YT-KIR2DL1 cells was assessed by staining with antibodies for IFN $\gamma$ (XMG1.2) and KIR2DL1 (HP-MA4). In addition, controls where the empty-vector transduced 721.221 cells or non-transduced 721.221 cells were co-cultured with YT-KIR2DL1 cells, or where YTKIR2DL1 cells were cultured with PMA and Calcium Ionomycin were included.

\section{Isolation and culture of trophoblast cells}

Trophoblast cells were isolated from implantation sites from age-matched pregnant female mice at gd12.5 as previously described (Rodrigues-Duarte et al., 2018). All placentas from the litter from a mouse were pooled and minced in RPMI. For every 6-8 placentas, $25 \mathrm{ml}$ digestion buffer containing HEPES (20 mM) (Gibco), sodium bicarbonate ( $0.35 \mathrm{~g} / \mathrm{L})$ (Gibco), collagenase type I ( $1 \mathrm{mg} / \mathrm{ml})$ (SigmaAldrich), DNAse I (20 $\mu \mathrm{g} / \mathrm{ml})$ in RPMI supplemented with Glutamax was used and incubated at $37^{\circ} \mathrm{C}$ for 1 hour. Cells were centrifuged at $500 \mathrm{~g}$ for $5 \mathrm{~min}$, and the pellet was re-suspended in $4 \mathrm{ml}$ of $25 \%$ Percoll and layered on top of $4 \mathrm{ml}$ of $40 \%$ Percoll, followed by centrifugation at $850 \mathrm{~g}$ for $20 \mathrm{~min}$ at $4{ }^{\circ} \mathrm{C}$ without brake. Trophoblast cells were removed from the interface, washed with PBS, incubated with ACK lysis buffer followed by another wash in PBS. Cells were plated at $1 \times 10^{5}$ cells/well in 96-well Ubottom plates and cultured in RPMI supplemented with Glutamax, 10\% heat inactivated FCS, sodium

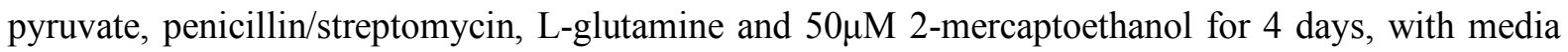
replenished after 2 days. On day 3, half the cells were stimulated with LPS $(10 \mu \mathrm{g} / \mathrm{ml})$ and IFN- $\gamma$ $(500 \mathrm{U} / \mathrm{ml})$, and the other half were cultured without stimulus. Cells were then harvested using Accutase cell dissociation reagent (Fisher Scientific) after 24 hours and stained as per the flow cytometric staining protocol. 


\section{ELISA}

Mouse VEGF R1/Flt-1 (R\&D systems), PIGF-2 (R\&D systems) and Endoglin/CD105 (R\&D systems) were measured in plasma samples collected from pregnant mice on gd18.5. For collection of plasma, murine blood was collected in EDTA-coated tubes followed by centrifugation at 2000g for $15 \mathrm{~min}$ at room temperature. Supernatants (plasma) were collected, aliquoted and stored at $-80^{\circ} \mathrm{C}$. Urine samples were also collected from pregnant female mice at gd18.5 (just prior to culling the mice for measurement of fetal weights), and aliquoted and stored at $-80^{\circ} \mathrm{C}$. Albumin and creatinine concentrations in the urine were determined by an Albumin ELISA (Bethyl Laboratories) and a Creatinine colorimetric assay (Cayman Chemical), respectively. The ratio of urinary albumin to creatinine was calculated and considered as a measure of proteinuria.

\section{RNA isolation, cDNA preperation and Q-PCR}

Total RNA was isolated from cells using the RNeasy isolation kit (Qiagen), and cDNA prepared using the Quantitech Reverse Transcription kit (Qiagen). SYBR-green based quantitative PCR assays were deigned and optimised for HLA-C and Hprt. Samples collected from tissue such as dissected implantation sites from pregnant mice or organs were cut into small pieces and immersed in RNAlater (Qiagen) as per manufacturer's instructions, prior to processing for RNA isolation. The sequences of the primers used for the assays are provided in the Table S3. Standard curves for each of the assays were performed using serial dilutions of cDNA and amplification efficiencies were determined. Relative expression was expressed as $2^{-\Delta \mathrm{Ct}}$, where $\Delta \mathrm{Ct}$ is the difference of the cycle threshold between the transcript of the gene of interest and the reference gene transcript.

\section{RNA in situ hybridization (RNA-ISH)}

RNA-ISH was performed on mouse implantation site tissues isolated from pregnant mice on gd9.5. Implantation sites were embedded in OCT and snap frozen in isopentane and dry ice. Serial $10 \mu \mathrm{m}$ coronal sections were cut on a cryostat, kept at $-20^{\circ} \mathrm{C}$ to dry for an hour and then stored at $-80^{\circ} \mathrm{C}$. Sections were hybridized with mRNA probes specific for the $C c l 1$, Gzme/d/g and Anxa2 genes (ACD). Following probe hybridization and amplification, mRNA was detected using the RNAscope $2.5 \mathrm{HD}$ 
Duplex Assay (ACD), as per the manufacturer's instructions. For quantification of stainings, tissue from four independent implantation sites were used per mouse group with multiple sections from the same region of the tissue used from each implantation site. Image analysis was completed by the Pharma Services group at Indica Labs (Albuquerque, NM, USA). Slides were scanned at 40X magnification on the Aperio Scanscope Turbo AT2 Slide Scanner (Leica Biosystems) and digital images imported into HALO $^{\circledR}$ Image Analysis Platform (Indica Labs, Albuquerque, NM USA). Collaboration between the two groups occurred through the use of a cloud-based $\mathrm{HALO}^{\circledR}$ Link Image Management system. Image analysis was based on teal-red duplex RNAScope ${ }^{\circledR}$ images counterstained with hematoxylin (SigmaAldrich) for nuclei detection. $\mathrm{HALO}^{\circledR}$ Link was utilized to import sample metadata and for researcher annotation of samples for regions of interest (ROIs). Annotations were used to identify individual tissue sections on separate layers of analysis. Multiple $\mathrm{HALO}^{\circledR}$ algorithms were utilized for analysis: ISH v3.3.9 (RNA probe identification) and Multiplex IHC v2.1.1 (cytoplasmic and nuclear staining localisation). The ISH algorithm was optimized to detect positive intensity of individual "spot-like" probes of Anxa2 and Ccl1. Anxa2 displayed the expected "spot-like" staining pattern of RNAScope ${ }^{\circledR}$ while Ccll displayed a stronger "spot-like" staining pattern than whole cell or nuclear staining, and both of these were accurately identified using ISH. In addition to the ISH algorithm we also utilized our Multiplex IHC algorithm because the staining pattern for Gzme/d/g was not a typical probe-like RNA stain. Therefore, the Multiplex IHC algorithm more accurately quantified this probe. The Multiplex IHC algorithm was optimized to detect positivity within the nuclear compartment of Gzme/d/g. The ISH module calculated the Probe Copies/cell for Anxa2 and Ccll, and the Multiplex IHC module calculated the overall \% positive cells for $\mathrm{Gzme} / \mathrm{d} / \mathrm{g}$.

\section{Blood pressure measurement in mice}

Systolic blood pressure was measured in mice using a non-invasive blood pressure analyser, BP-2000 Blood Pressure Analysis System (Bioseb), that uses tail-cuff transmission photoplethysmography. The restraint platform was kept at a temperature of $37^{\circ} \mathrm{C}$. Baseline blood pressure measurements for each mouse were taken prior to using the mice for timed-matings. Baseline measurements required recording of valid measurements for at least 3 days for each mouse with a minimum of 4 valid readings on each 
day of measurement. During the course of gestation, measurements were taken every alternate day from $\operatorname{gd} 2.5$ until gd14.5 and then every day from gd15.5 until gd18.5. Post-partum measurements were taken daily from day 1 post-partum until 6-7 days post-partum. Inclusion of measurements during pregnancy and post-partum for each mouse required recording of a minimum of 4 valid readings on each day of measurement. Collected data were analysed with the BP-2000 Analysis Software (Bioseb) and the change in blood pressure for each mouse was normalised to its baseline.

\section{Perfusion of uteroplacental vasculature for Micro-CT imaging and vascular segmentation analysis}

The method for injection of the silicon rubber injection compound, Microfil (Flow Tech) into the uteroplacental vasculature of mice for micro-CT imaging has been previously described (Rennie et al., 2014). In brief, pregnant mice at gd10.5 were given terminal anaesthesia using pentobarbitone and intracardiac perfusion was performed using a Heparin solution (100 IU/ml) in PBS. A catheter was placed in the descending thoracic aorta and used to clear the lower body vasculature of blood and perfused with heparinised PBS. The contrast agent, Microfil was then gently infused by hand on a slow flow rate until the blue colour of the contrast agent was visible through the capillary bed and sufficiently vented through the right atrium. The inferior vena cava was then immediately tied off and the vasculature was kept pressurised using a syringe that contained the contrast agent to sustain vessel inflation, until the Microfil polymerised. The uterus was then removed, immersed in formalin (SigmaAldrich) overnight and the implantation sites were mounted in $1 \%$ agarose for micro-CT imaging. Micro-CT imaging was performed on Skyscan 1172 (Bruker) at $40 \mathrm{kV}, 250 \mu \mathrm{A}, 750 \mathrm{~ms}$ exposure time and a pixel size of $4.73 \mu \mathrm{m}$, and the three-dimensional (3D) images were reconstructed using Nrecon (Micro Photonics). As the uteroplacental vasculature was difficult to segment automatically due to the unique nature of the tightly coiled spiral arteries, the micro-CT datasets were manually segmented slice by slice using the seg3D software ("Seg3D"). Each acquired image stack was first downscaled by $50 \%$ (to typical dimensions 900x900x1000) and 3D median filtering was applied (radius 2 voxels, isotropic). Each voxel of the resulting stack was classified as background, vessel or transitional using multi-Otsu 
thresholding. With the aid of these pre-assigned non-overlapping classes, assisted manual segmentation was performed using seg3 $\mathrm{D}$, segmenting the volume into spiral arteries, radial arteries, maternal canals, uterine arteries, and background. The first step of the automated analysis was to translate the voxelbased information in the segmented image stacks into a graph structure that is better suited for quantification algorithms. The uterine, spiral, radial and maternal canal vessels were combined into a single class and skeletonisation was performed using homotopic thinning (Lee et al., 1994). The skeletonised network was then organised into segments, where a segment denotes the ordered chain of vertices that run between two adjacent junctions, or a junction and a terminal. Spurious segments that may result from noisy segmentation were detected using a custom routine and removed from the network (Lee et al., 2007). All subnetworks (a collection of segments that are connected to each other) were detected and the smallest subnetworks below the threshold ( $<30$ segments) were removed. Vessel class labelling from the segmentation was automatically transferred to the segments. Because the skeletonisation process places nodes at discrete voxel centres, the total number of nodes in a given network depended on the total length of the vessels in it. To allow inter-sample comparisons, we chose to normalise the number of total nodes in each network to 10,000 by resampling along each segment. The distance between each adjacent pair of nodes was kept approximately constant, subject to the constraint of a discrete number of voxels along a segment. At each node, the local radius of the vessel segment was estimated using Rayburst algorithm (Rodriguez et al., 2006). Furthermore, to refine the voxel-based quantification of radius, a sequence of synthetic vessel-like image stacks with known radii were created and nonlinear regression was used to calibrate the Rayburst estimates into sub-voxel quantities. Using the Rayburst kernel, the location of each skeleton node was also adjusted towards the true vessel centreline on a sub-voxel basis, to reduce the staircase artefact. Vessel morphological quantifications were performed either on a nodal basis for radii calculations, or segmental basis for calculation of length and volume, for vessels $>10 \mu \mathrm{m}$ in radius and length. For segmental quantification, the summed segment length and volume were calculated using a piecewise linear approximation. Flow conductance of each segment was calculated as the reciprocal of Poiseuille resistance, under the assumption that the flow will remain laminar. Given the typical inlet vessel dimensions, this assumption was regarded as appropriate. However, the calculation of total spiral arteries conductance requires 
further assumptions about boundary conditions. For this, the terminal vessels of the spiral subnetwork were detected and their proximity to the radial or canal vessels were evaluated, thereby classifying every terminal as being radial- or canal-adjacent. All radial-connected spiral arteries were assumed as flow inlets and all canal-connected arteries, as outlets. In general, the number of inlets and outlets are not consistent between different samples. We calculated the total spiral network resistance by imposing a pressure gradient between the inlets and outlets of $1 \mathrm{kPa}$ and calculating the total outflow using a network-Poiseuille formulation, as $R=\Delta P / Q$. Due to the linearity of the flow model, the resistance remains constant regardless of the actual pressure gradient applied. For analysis, flow conductance is quoted, which are reciprocal of the resistance (the greater the conductance, the easier the fluid to flow).

\section{Isolation of cells from implantation sites and FACS sorting for scRNA-seq studies}

Isolation of cells from the implantation sites were done with enzymatic digestion using Liberase ${ }^{\mathrm{TM}}$ as previously described (Collins et al., 2009). In brief, uterine horns were collected in cold HBSS containing $\mathrm{Ca}^{2+}$ and $\mathrm{Mg}^{2+}$ (ThermoFisher) from pregnant mice and cleared of mesometrial fat. The tissue was collected from the entire mesometrial side of the implantation site leaving behind the developing fetus (Edwards et al., 2014; Pang et al., 2014). All implantation sites from a litter were pooled and processed together. The tissue was finely minced, incubated in HBSS solution containing 0.28 Wunsch Units Liberase ${ }^{\mathrm{TM}}$ and $30 \mu \mathrm{g} / \mathrm{ml}$ DNase for 30 minutes at $37^{\circ} \mathrm{C}$ with agitation, washed with $\mathrm{Ca}^{2+} \mathrm{Mg}^{2+}$-free PBS containing 5mM EDTA (ThermoFisher) and centrifuged. Cells were incubated in $\mathrm{Ca}^{2+} \mathrm{Mg}^{2+}$-free PBS containing 5mM EDTA for $15 \mathrm{~min}$ at $37^{\circ} \mathrm{C}$, followed by filtering through a $70 \mu \mathrm{m}$ cell strainer, red blood cell lysis and washing in PBS. Cells were then filtered again through a $70 \mu \mathrm{m}$ cell strainer and counted.

For isolation of total unsorted cells from the implantation site, half of the isolated cells were taken and depleted of dead cells by labelling with the Dead cell removal microbeads (Miltenyi Biotech) as per the manufacturer's instructions. The viable cell fraction eluted from the MS columns (Miltenyi Biotech) was counted and processed for droplet based scRNA-seq (below). 
For isolation of uNK cells, half of the isolated cells were surface stained using the flow cytometric staining protocol for antibodies against CD45 (30-F11), CD3 (145-2C11), CD19 (eBio1D3), TCRb (H57-597), NKp46 (29A1.4), CD49a (Ha31/8), CD49b (DX5), KIR2DL1 (HP-MA4), CD122 (TM-b1) along with either 7AAD or a Live/Dead viability dye. For SMART-Seq2 processing, single cells were FACS sorted into $96 \mathrm{U}$-well plates into either trNK (NCR1 ${ }^{+} \mathrm{CD} 3 \varepsilon^{-} \mathrm{TCRb}^{-} \mathrm{CD} 19-\mathrm{CD} 45^{+} \mathrm{CD} 122^{+} \mathrm{CD}^{-} 4 \mathrm{a}^{+}$) or $\mathrm{cNK}\left(\mathrm{NCR} 1{ }^{+} \mathrm{CD} 3 \varepsilon^{-} \mathrm{TCRb}{ }^{-\mathrm{CD}} 19^{-} \mathrm{CD} 45^{+} \mathrm{CD} 122^{+} \mathrm{CD} 49 \mathrm{~b}^{+}\right.$) cells. Single cells were directly sorted into wells containing $5 \mu \mathrm{l}$ of pre-chilled TCL buffer (Qiagen) containing 1\% 2-mercaptoethanol as previously described (Trombetta et al., 2014). Each plate consisted of a negative control with no cells and a positive control with 15 cells sorted in one well. 96-well plates containing the sorted cells were then immediately centrifuged and frozen directly on dry ice prior to storage at $-80^{\circ} \mathrm{C}$. For droplet based scRNA-seq, total uNK cells $\left(\mathrm{NCR} 1^{+} \mathrm{CD} 3 \varepsilon^{-} \mathrm{TCRb}{ }^{-\mathrm{CD}} 19^{-\mathrm{CD}} 45^{+} \mathrm{CD} 122^{+}\right)$were sorted into Eppendorf tubes containing PBS with $0.04 \%$ BSA and counted and processed as described below.

\section{Full length scRNA-Seq}

SMART-Seq2 scRNA-seq libraries were prepared from trNK and cNK cells sorted in 96 U-well plates as previously described (Picelli et al., 2014; Trombetta et al., 2014) with modifications in the protocol as previously described (Ding et al., 2020). Briefly, RNA was purified from single cell lysates using 2.2x RNAClean XP beads (Beckman Coulter), eluted in $4 \mu 1$ of master mix containing $1 \mu 1$ of $10 \mathrm{mM}$ dNTPs (ThermoFisher), $0.1 \mu 1$ of $100 \mu \mathrm{M}$ 3' oligodT RT primer, $0.1 \mu 1$ of $40 \mathrm{U} / \mu 1$ RNase Inhibitor (Clontech Takara) and $2.8 \mu 1$ of $1 \mathrm{M}$ Trehalose (Life Sciences Advanced Technologies), followed by incubation at $72^{\circ} \mathrm{C}$ for $3 \mathrm{~min}$ and placing on ice. The remaining reverse transcription mix, containing $2 \mu 1$ of Maxima RT buffer (ThermoFisher), $0.1 \mu \mathrm{l}$ of $100 \mu \mathrm{M}$ TSO oligo, $0.25 \mu \mathrm{l}$ of $40 \mathrm{U} / \mu 1 \mathrm{RNase}$ Inhibitor, $0.1 \mu 1$ of $200 \mathrm{U} / \mu 1$ Maxima H Minus Reverse Transcriptase (ThermoFisher), $3.45 \mu 1$ of $1 \mathrm{M}$ Trehalose and $0.1 \mu 1$ of $1 \mathrm{M} \mathrm{MgCl}_{2}$ (Sigma-Aldrich) was added to each well and incubated for $50^{\circ} \mathrm{C}$ for 90 min followed by $85^{\circ} \mathrm{C}$ for $5 \mathrm{~min}$. The cDNA was then amplified using $12.5 \mu 1$ of KAPA HiFi HotStart ReadyMix (KAPA Biosystems) and $0.05 \mu$ of $100 \mu \mathrm{M}$ ISPCR primer and incubated at $98^{\circ} \mathrm{C}$ for $3 \mathrm{~min}$ followed by 23 cycles of $98^{\circ} \mathrm{C}$ for $15 \mathrm{sec}, 67^{\circ} \mathrm{C}$ for $20 \mathrm{sec}, 72^{\circ} \mathrm{C}$ for $6 \mathrm{~min}$, and a final extension at $72^{\circ} \mathrm{C}$ for 5 min. PCR products were purified using 0.7x AMPure XP SPRI beads (Beckman 
Coulter) and eluted in $20 \mu \mathrm{l}$ of TE buffer (Qiagen). DNA quantification was done using the Qubit ${ }^{\mathrm{TM}}$ dsDNA HS Assay Kit (ThermoFisher) and DNA fragment size was assessed using the High Sensitivity DNA BioAnalyzer kit (Agilent). cDNA concentrations were normalised and $0.125 \mathrm{ng}$ of each cDNA was used in a quarter volume of a Nextera XT DNA library preparation kit (Illumina), following pooling of equal volumes from each well and purification with 0.6x AMPure XP SPRI beads. Final libraries were again assessed for DNA concentration using the Qubit assay and fragment size using the Bioanalyzer. All SMART-Seq2 Libraries were sequenced using a NextSeq 500/550 High Output v2 kit 75 cycles (Illumina) on a Nextseq 500 (Illumina).

\section{Droplet based scRNA-Seq}

For droplet based scRNA-seq, 7,000 cells were loaded onto the Chromium Single Cell 3' Chip A (10x Genomics) and processed through the chromium controller to generate Gel beads in Emulsion. RNAseq libraries were prepared using the Chromium Single Cell 3' Library \& Gel Bead Kit v2 (10x Genomics) as per the manufacturer's instructions. Libraries were sequenced on an Illumina Hi-Seq.

\section{Statistical analysis}

Data were analysed using Prism 8 or RStudio. Statistical details of experiments including sample size can be found in the figure legends. To statistically estimate the effect of genotype on fetal or placental weight, a linear mixed-effects model was used with transgenic genotype mating combination as a fixed effect and clustering of observations within a litter as a random effect. Similarly, for estimation of the effect of genotype on spiral artery diameter and segment length, a linear mixed-effects model was used with transgenic genotype mating combination as a fixed effect and clustering of observations from a specific implantation site as well as clustering of observations within a litter from a mouse as random effects. As the segment length data followed a log-normal distribution, the mixed effects model for assessing effect on segment length was run on log-transformed length values. For comparison of twogroup datasets with non-Gaussian distribution, the non-parametric Mann-Whitney test was used. Values were expressed as mean \pm S.E.M. P values lower than 0.05 were considered statistically significant. For all the statistical analysis: ${ }^{*} \mathrm{p}<0.05, * * \mathrm{p}<0.01, * * * \mathrm{p}<0.001, * * * \mathrm{p}<0.0001$ 


\section{scRNA-Seq data preprocessing}

For full length scRNA-Seq SMART-Seq2 data, raw sequencing data was demultiplexed to fastq files using bcl2fastq (v.2.17.1.14) and aligned to a custom reference using RSEM (v.1.2.8); the custom reference included the mm10 genome and the sequence for the human genes KIR2DL1 and HLA-C*05 (as described in the transgenic mice generation above), and was generated using rsem-preparereference. Quality control was assessed and summaries generated using STAR. We used RSEM (v1.2.8) to quantify gene counts and TPM (we converted TPM to TP100K after initial quality control filtering, described below).

For droplet-based scRNA-Seq data, raw sequencing data was demultiplexed to fastq files with cellranger mkfastq (version 2.1.0, 10x Genomics) and reads were aligned and count matrices were generated with cellranger count (version 2.0.1). A custom reference was generated to include the $H L A$ $C^{*} 05$ and KIR2DL1 transgenic construct sequences into the mm10 reference using cellranger mkref (version 2.1.0). The alignment of $H L A-C^{*} 05$ sequence was limited to the human DNA sequence region to minimise the risk of misalignment with the mouse $H-2 K^{b}$ gene.

\section{scRNA-Seq quality control}

For full length scRNA-seq, lower-quality cells were removed if they met any of the following criteria: (1) $\log _{10}$ (counts) $<5$, (2) number of expressed genes $<1,000$ or $>7,000$, or (3) average housekeeping gene (Table S1) expression $(\mathrm{TPM})>1$. In addition, cells that met any of the following criteria were also removed (to ensure cells meet FACS expectations): (1) Cells with HLA-C*05 expression $<2$ in $\mathrm{C}^{*} 05 / \mathrm{KIR} \times \mathrm{x}$ W mating group; (2) Cells with HLA-C*05 expression $>2$ in WT x $\mathrm{C} * 05$ mating group, (3) Cells with KIR2DL1 expression $<2$ in KIR x C*05 and C*05/KIR x WT mating groups; (4) Cells with KIR2DL1 expression > 2 in WT $x \mathrm{C}^{*} 05$ mating group; (5) Cells with Cd3e expression > 3; (6) In sorted cNK cells, cNK cells with Itgal expression $>1$. Genes expressed in three or fewer cells were removed. Final expression values were normalized to TP100K and log transformed. 
For droplet-based scRNA-seq, the feature-barcode matrix cellranger count was used, but with forcecells $=6000$ to ensure all droplets containing cells are included. Low quality cells were removed by filtering any cells with either (1) less than 500 genes, (2) less than 1000 UMIs, or (3) more than 10\% of UMIs mapped to mitochondrial genes. Genes expressed in three or fewer cells were removed. The final expression values were normalized to a total UMI per cell of $1 \times 10^{4}$ and log transformed.

We used Scrublet (v.0.2.1), with expected_doublet_rate $=0.06$, to predict doublets (Wolock et al., 2019). In the NK cell sorted droplet-based data we removed one cluster with high doublet probability as well as one cluster of non-NK cells, and then re-analyzed the remaining data. In the unsorted dropletbased data we excluded from visualization and analysis cells from two clusters with high doublet scores, but did not reanalyze the data following exclusion.

\section{Dimensionality reduction, clustering and visualization}

In both full length and droplet-based scRNA-seq, human genes were removed from the expression matrix prior to dimensionality reduction. We did not batch-correct by mouse because mice and genotype were evenly distributed across clusters (Fig. S4C,F,I,L,S7E, adjusted rand index between sample and cluster $=0.49 \%$ for full length NK data, $0.88 \%$ for droplet-based NK data, and $1.21 \%$ for the dropletbased unsorted data).

For both the full-length and droplet-based scRNA-seq data, highly variable genes were selected using FindVariableGenes (default parameters) in Seurat (Butler et al., 2018) [v.2.1]. For the full-length data, the expression of each gene was centered and scaled to have a mean of zero and a standard deviation of one; for droplet-based data, the expression of each gene was centered to have a mean of zero.

In both full length and droplet-based data sets, initial dimensionality reduction was performed by principal component analysis (PCA) using RunPCA in Seurat (v.2.1) (Butler et al., 2018), and Louvain graph-based clustering was performed using FindClusters (based on the top 15 PCs, with clustering resolutions of $0.8,0.4$, and 0.6 for the full-length $\mathrm{NK}$, droplet-based NK, and unsorted droplet-based 
data, respectively; other parameters, including $k=30$ for $k$-NN, were set to the default). Cell profiles were visualized using a Uniform Manifold Approximation and Projection (UMAP) (McInnes et al., 2018) embedding of the top 15 PCs, with min_dist $=0.5$, spread=1.0, number of neighbors $=15$, and the Euclidean distance metric.

The statistical significance of genotype proportions within each cluster was tested using a Dirichletmultinomial regression (Smillie et al., 2019). Mouse batch effects were assessed within each cluster by calculating the rand index using the Clues package in $\mathrm{R}$.

\section{Differentially expressed genes, annotating cell subsets, and building gene signatures}

Subset-specific marker genes (all data sets) and cNK/trNK-specific (full-length data) differentially expressed genes were identified by a Mann-Whitney U test using the FindAllMarkers function in Seurat (v.2.1), with test.use $=$ 'wilcox', $\log f$ c.threshold $=\log (1.5)$ and min.pct $=0.2$; the Benjamini-Hochberg False Discovery Rate (FDR) was calculated using p.adjust in R (method = 'Benjamini \& Hochberg'). Cell subsets were manually annotated using cell subset marker genes and expression of relevant published signatures (Aran et al., 2017; Nelson et al., 2016; Pavlicev et al., 2017; Tirosh et al., 2016; Tsang et al., 2017; Vento-Tormo et al., 2018; Wallrapp et al., 2017). For the two NK cell scRNA-seq data sets (full-length and droplet-based) and some cell subsets from the unsorted droplet-based data, novel cell subsets are described by their expressed marker genes.

Within each subset, we tested for differentially expressed genes between IUGR and CTR1, IUGR and CTR2, and CTR1 and CTR2 using: (1) a pseudobulk differential expression analysis and (2) a mixedeffects Poisson regression model. In both approaches, genes were only tested for differential expression if they were expressed in at least $10 \%$ of cells within one of the tested conditions for a given cell subset. For the pseudobulk approach, we summed counts per mouse within a subset and then performed differential expression analysis using DESeq2 (v.1.26, design formula: gene $\sim$ condition) (Love et al., 2014). We used the fdrtools (v.1.2.16) package (Strimmer, 2008) in R to calculate FDR based on the empirical null, with the DESeq2 Wald statistic as input. For the mixed-effects Poisson regression model 
(Subramanian et al., 2020), we used the glmer function from the lme4 package (v.1.1-26) (Bates et al., 2015 ) in $\mathrm{R}$ with family = poisson() and the following design formula: gene $\sim$ condition + offset(log(total_UMI/mean_UMI)) +(1|mouseID). We calculated FDR using p.adjust in R (method = 'Benjamini \& Hochberg'). IUGR-unique expression changes were defined as genes that were differentially expressed in the IUGR vs. CTR1 (FDR $<0.05$ ), but not differentially expressed in CTR1 vs. CTR2 (FDR <0.05). Volcano plots of differentially expressed genes were made using the R package, EnchancedVolcano (v.1.4.0) (Blighe et al., 2019).

Gene signatures for the cNK and trNK cells and for NK cell subsets were defined as the union of the top 25 differentially expressed genes (sorted by p-value) and the top 25 differentially expressed genes sorted by log fold change. The proliferation signature was previously reported (Wallrapp et al., 2017). Gene signatures were scored in each cell using AddModuleScore in Seurat (Butler et al., 2018) (v.2.1 for scoring proliferation and v.3.2.2 for scoring NK cell subsets).

\section{Topic modeling}

Topic models were fit to the droplet-based sorted uNK UMI counts using the FitGoM() function from the CountClust (v.1.6.1) R package (Bielecki et al., 2018; Dey et al., 2017; Xu et al., 2019). To select the number of topics, $K$, models are fit for each of a range of $K(\mathrm{~K}=4: 22$ in increments of two) with a tolerance parameter (tol) of tol $=0.1$ and tol $=0.01$. The Bayesian Information Criterion (BIC), estimated likelihood, and Akaike Information Criterion (AIC) were computed using compGoM() for each model. The final value of $\mathrm{K}(\mathrm{K}=16)$ was chosen by considering the BIC curve (seeking a $\mathrm{K}$ for which the BIC was either minimizing or decreasing slowly) and the biological relevance of the identified gene programs. The final value of tol $=0.01$ was chosen primarily by the biological relevance of the identified gene programs. Top scoring genes per topic were defined using the extractTopFeatures function.

Topics significantly changed in IUGR were selected by examining four criteria: (1) the sign of the KS statistic; (2) the Benjamini Hochberg FDR following the KS-test; (3) the fold change direction and magnitude of the mean; and (4) the fold change direction and magnitude of the median. The ks.test and 
p.adjust (with method $=$ 'Benjamini \& Hochberg') functions in $\mathrm{R}$ were used to calculate the FDR for IUGR vs. CTR1 and IUGR vs. CTR2 under the KS test. The KS-statistic was calculated with the ks.test function in $\mathrm{R}$ as cumsum(ifelse(order(w) $<=n . x, 1 / n \cdot x,-1 / n \cdot y)$ ), where $n . x=$ length(x), n.y = length(y), and $w=c(x, y)$. The final statistic was reported as the maximum or minimum of the above equation, whichever had the larger absolute magnitude. We examined the above four criteria using $100 \%$ of cells as well as cells with cell weights in the top $75 \%, 50 \%$, and $25 \%$ for each topic; we report metrics based on cells with weights in the top $75 \%$ as this retained the most number of cells without a skew towards cells with very low weights in a topic.

EnrichR (Chen et al., 2013; Kuleshov et al., 2016) and Ingenuity Pathway Analysis (Qiagen) were used to calculate pathway enrichment. Enrichment of canonical pathways, diseases and functions was performed in IPA using gene lists of IUGR-unique expression changes (defined as genes that were differentially expressed in IUGR vs. CTR1 but not differentially expressed in CTR1 vs. CTR2 within each subset; above); the background was the intersection of genes that were in IPA with the genes that were expressed in the cell subset in at least $10 \%$ of the cells from either condition.

\section{Cell-cell interactions}

Mouse genes were mapped to their human genes orthologs using the Human Phenotype Ontology(http://www.informatics.jax.org/downloads/reports/HMD_HumanPhenotype.rpt).

CellPhoneDB (Vento-Tormo et al., 2018) was then used to identify individual significant ligandreceptor interactions between subsets in the unsorted droplet-based scRNA-Seq data with cellphonedb method statistical_analysis meta.txt counts.txt - -result-precision $=3$--iterations $=1000$, where meta.txt is cell subset assignments, counts.txt is the normalized counts data for either the WT x $\mathrm{C}^{*} 05$ or KIR $\mathrm{x}$ $C^{*} 05$ matings, result-precision $=3$ indicated the number of decimal points for the reported p-value, and iterations was the number of iterations for the statistical test.

The number of significant interactions was summed for each subset pair, and interactions were visualized using Cytoscape v3.8.2 (Shannon et al., 2003). Only subset pairs with at least 50 interactions 
were connected on the Cytoscape map, and line thickness corresponded to the number of significant interactions.

\section{Data and code availability}

Raw data, gene expression matrices and the code used to generate results presented in this paper will be made available upon publication 


\section{Methods References}

"Seg3D”, S.C.a.I.I. "Seg3D" Volumetric Image Segmentation and Visualization. Scientific Computing and Imaging Institute (SCI).

Adamson, S.L., Lu, Y., Whiteley, K.J., Holmyard, D., Hemberger, M., Pfarrer, C., and Cross, J.C. (2002). Interactions between trophoblast cells and the maternal and fetal circulation in the mouse placenta. Dev Biol 250, 358-373.

Allen, M.P., and Nilsen-Hamilton, M. (1998). Granzymes D, E, F, and G are regulated through pregnancy and by IL-2 and IL-15 in granulated metrial gland cells. J Immunol 161, 2772-2779.

Apps, R., Murphy, S.P., Fernando, R., Gardner, L., Ahad, T., and Moffett, A. (2009). Human leucocyte antigen (HLA) expression of primary trophoblast cells and placental cell lines, determined using single antigen beads to characterize allotype specificities of anti-HLA antibodies. Immunology 127, 26-39.

Aran, D., Hu, Z., and Butte, A.J. (2017). xCell: digitally portraying the tissue cellular heterogeneity landscape. Genome Biol 18, 220.

Ashkar, A.A., Black, G.P., Wei, Q., He, H., Liang, L., Head, J.R., and Croy, B.A. (2003). Assessment of requirements for IL-15 and IFN regulatory factors in uterine NK cell differentiation and function during pregnancy. J Immunol 171, 2937-2944.

Ashkar, A.A., Di Santo, J.P., and Croy, B.A. (2000). Interferon gamma contributes to initiation of uterine vascular modification, decidual integrity, and uterine natural killer cell maturation during normal murine pregnancy. J Exp Med 192, 259-270.

Babic, A.M., Kireeva, M.L., Kolesnikova, T.V., and Lau, L.F. (1998). CYR61, a product of a growth factor-inducible immediate early gene, promotes angiogenesis and tumor growth. Proc Natl Acad Sci U S A 95, 6355-6360.

Bashirova, A.A., Martin, M.P., McVicar, D.W., and Carrington, M. (2006). The killer immunoglobulin-like receptor gene cluster: tuning the genome for defense. Annu Rev Genomics Hum Genet 7, 277-300.

Bates, D., Mächler, M., Bolker, B., and Walker, S. (2015). Fitting Linear Mixed-Effects Models Using lme4. 2015 67, 48.

Bernstein, I.M., Horbar, J.D., Badger, G.J., Ohlsson, A., and Golan, A. (2000). Morbidity and mortality among very-low-birth-weight neonates with intrauterine growth restriction. The Vermont Oxford Network. Am J Obstet Gynecol 182, 198-206.

Bielecki, P., Riesenfeld, S.J., Kowalczyk, M.S., Amezcua Vesely, M.C., Kroehling, L., Yaghoubi, P., Dionne, D., Jarret, A., Steach, H.R., McGee, H.M., et al. (2018). Skin inflammation driven by differentiation of quiescent tissue-resident ILCs into a spectrum of pathogenic effectors. bioRxiv.

Blighe, K., Rana, S., and Lewis, M. (2019). EnhancedVolcano: Publication-ready volcano plots with enhanced colouring and labeling. R.

Borenstein, S.H., Graham, J., Zhang, X.L., and Chamberlain, J.W. (2000). CD8+ T cells are necessary for recognition of allelic, but not locus-mismatched or xeno-, HLA class I transplantation antigens. J Immunol 165, 2341-2353.

Bu, P., Alam, S.M., Dhakal, P., Vivian, J.L., and Soares, M.J. (2016). A Prolactin Family Paralog Regulates Placental Adaptations to a Physiological Stressor. Biol Reprod 94, 107. Burton, G.J., and Jauniaux, E. (2018). Pathophysiology of placental-derived fetal growth restriction. Am J Obstet Gynecol 218, S745-S761.

Butler, A., Hoffman, P., Smibert, P., Papalexi, E., and Satija, R. (2018). Integrating singlecell transcriptomic data across different conditions, technologies, and species. Nat Biotechnol $36,411-420$. 
Carmon, K.S., and Loose, D.S. (2008). Secreted frizzled-related protein 4 regulates two Wnt7a signaling pathways and inhibits proliferation in endometrial cancer cells. Mol Cancer Res 6, 1017-1028.

Chen, C.M., Krohn, J., Bhattacharya, S., and Davies, B. (2011). A comparison of exogenous promoter activity at the ROSA26 locus using a PhiiC31 integrase mediated cassette exchange approach in mouse ES cells. PLoS One 6, e23376.

Chen, E.Y., Tan, C.M., Kou, Y., Duan, Q., Wang, Z., Meirelles, G.V., Clark, N.R., and Ma'ayan, A. (2013). Enrichr: interactive and collaborative HTML5 gene list enrichment analysis tool. BMC Bioinformatics 14, 128.

Chiossone, L., Chaix, J., Fuseri, N., Roth, C., Vivier, E., and Walzer, T. (2009). Maturation of mouse NK cells is a 4-stage developmental program. Blood 113, 5488-5496.

Collins, M.K., Tay, C.S., and Erlebacher, A. (2009). Dendritic cell entrapment within the pregnant uterus inhibits immune surveillance of the maternal/fetal interface in mice. J Clin Invest 119, 2062-2073.

Conde-Agudelo, A., Papageorghiou, A.T., Kennedy, S.H., and Villar, J. (2013). Novel biomarkers for predicting intrauterine growth restriction: a systematic review and metaanalysis. BJOG 120, 681-694.

Croy, B.A., Ashkar, A.A., Minhas, K., and Greenwood, J.D. (2000). Can Murine Uterine Natural Killer Cells Give Insights Into the Pathogenesis of Preeclampsia? Journal of the Society for Gynecologic Investigation 7, 12-20.

Croy, B.A., McBey, B.A., Villeneuve, L.A., Kusakabe, K., Kiso, Y., and van den Heuvel, M. (1997). Characterization of the cells that migrate from metrial glands of the pregnant mouse uterus during explant culture. J Reprod Immunol 32, 241-263.

Davies, J.E., Pollheimer, J., Yong, H.E., Kokkinos, M.I., Kalionis, B., Knofler, M., and Murthi, P. (2016). Epithelial-mesenchymal transition during extravillous trophoblast differentiation. Cell Adh Migr 10, 310-321.

de Onis, M., Blossner, M., and Villar, J. (1998). Levels and patterns of intrauterine growth retardation in developing countries. Eur J Clin Nutr 52 Suppl 1, S5-15.

Delgado, S.R., McBey, B.A., Yamashiro, S., Fujita, J., Kiso, Y., and Croy, B.A. (1996). Accounting for the peripartum loss of granulated metrial gland cells, a natural killer cell population, from the pregnant mouse uterus. J Leukoc Biol 59, 262-269.

Demaison, C., Parsley, K., Brouns, G., Scherr, M., Battmer, K., Kinnon, C., Grez, M., and Thrasher, A.J. (2002). High-level transduction and gene expression in hematopoietic repopulating cells using a human immunodeficiency [correction of imunodeficiency] virus type 1-based lentiviral vector containing an internal spleen focus forming virus promoter. Hum Gene Ther 13, 803-813.

Dey, K.K., Hsiao, C.J., and Stephens, M. (2017). Visualizing the structure of RNA-seq expression data using grade of membership models. PLoS Genet 13, e1006599.

Diao, H., Xiao, S., Zhao, F., and Ye, X. (2010). Uterine luminal epithelium-specific prolinerich acidic protein 1 (PRAP1) as a marker for successful embryo implantation. Fertil Steril 94, 2808-2811 e2801.

Ding, J., Adiconis, X., Simmons, S.K., Kowalczyk, M.S., Hession, C.C., Marjanovic, N.D., Hughes, T.K., Wadsworth, M.H., Burks, T., Nguyen, L.T., et al. (2020). Systematic comparison of single-cell and single-nucleus RNA-sequencing methods. Nature Biotechnology.

Ding, J., Cheng, Y., Zhang, Y., Liao, S., Yin, T., and Yang, J. (2019). The miR-27a3p/USP25 axis participates in the pathogenesis of recurrent miscarriage by inhibiting trophoblast migration and invasion. J Cell Physiol 234, 19951-19963. 
Doisne, J.M., Balmas, E., Boulenouar, S., Gaynor, L.M., Kieckbusch, J., Gardner, L., Hawkes, D.A., Barbara, C.F., Sharkey, A.M., Brady, H.J., et al. (2015). Composition, Development, and Function of Uterine Innate Lymphoid Cells. J Immunol 195, 3937-3945. Donnelly, R.P., Sheikh, F., Kotenko, S.V., and Dickensheets, H. (2004). The expanded family of class II cytokines that share the IL-10 receptor-2 (IL-10R2) chain. J Leukoc Biol 76, 314-321.

Duhig, K.E., Myers, J., Seed, P.T., Sparkes, J., Lowe, J., Hunter, R.M., Shennan, A.H., Chappell, L.C., and group, P.t. (2019). Placental growth factor testing to assess women with suspected pre-eclampsia: a multicentre, pragmatic, stepped-wedge cluster-randomised controlled trial. Lancet 393, 1807-1818.

Dunn, C.L., Critchley, H.O., and Kelly, R.W. (2002). IL-15 regulation in human endometrial stromal cells. J Clin Endocrinol Metab 87, 1898-1901.

Eckelhart, E., Warsch, W., Zebedin, E., Simma, O., Stoiber, D., Kolbe, T., Rulicke, T., Mueller, M., Casanova, E., and Sexl, V. (2011). A novel Ncr1-Cre mouse reveals the essential role of STAT5 for NK-cell survival and development. Blood 117, 1565-1573. Edwards, A.K., Janzen-Pang, J., Peng, A., Tayade, C., Carniato, A., Yamada, A.T., Lima, P.D.A., and Tse, D. (2014). 3 - Microscopic Anatomy of the Pregnant Mouse Uterus Throughout Gestation. In The Guide to Investigation of Mouse Pregnancy, B.A. Croy, A.T. Yamada, F.J. DeMayo, and S.L. Adamson, eds. (Boston: Academic Press), pp. 43-67. Ego, A., Zeitlin, J., Batailler, P., Cornec, S., Fondeur, A., Baran-Marszak, M., Jouk, P.S., Debillon, T., and Cans, C. (2013). Stillbirth classification in population-based data and role of fetal growth restriction: the example of RECODE. BMC Pregnancy Childbirth 13, 182. Ek-Rylander, B., Flores, M., Wendel, M., Heinegard, D., and Andersson, G. (1994). Dephosphorylation of osteopontin and bone sialoprotein by osteoclastic tartrate-resistant acid phosphatase. Modulation of osteoclast adhesion in vitro. J Biol Chem 269, 14853-14856. Fang, F., Wang, Y., Li, R., Zhao, Y., Guo, Y., Jiang, M., Sun, J., Ma, Y., Ren, Z., Tian, Z., et al. (2010). Transcription factor E2F1 suppresses dendritic cell maturation. J Immunol 184, 6084-6091.

Fenouille, N., Puissant, A., Tichet, M., Zimniak, G., Abbe, P., Mallavialle, A., Rocchi, S., Ortonne, J.P., Deckert, M., Ballotti, R., et al. (2011). SPARC functions as an anti-stress factor by inactivating p53 through Akt-mediated MDM2 phosphorylation to promote melanoma cell survival. Oncogene 30, 4887-4900.

Figueras, F., and Gardosi, J. (2011). Intrauterine growth restriction: new concepts in antenatal surveillance, diagnosis, and management. Am J Obstet Gynecol 204, 288-300.

Forsen, T., Eriksson, J., Tuomilehto, J., Reunanen, A., Osmond, C., and Barker, D. (2000). The fetal and childhood growth of persons who develop type 2 diabetes. Ann Intern Med 133, 176-182.

Frankel, S., Elwood, P., Sweetnam, P., Yarnell, J., and Smith, G.D. (1996). Birthweight, body-mass index in middle age, and incident coronary heart disease. Lancet 348, 1478-1480. Fu, B., Zhou, Y., Ni, X., Tong, X., Xu, X., Dong, Z., Sun, R., Tian, Z., and Wei, H. (2017). Natural Killer Cells Promote Fetal Development through the Secretion of Growth-Promoting Factors. Immunity 47, 1100-1113 e1106.

Gao, S., Zhu, H., Zuo, X., and Luo, H. (2018). Cathepsin G and Its Role in Inflammation and Autoimmune Diseases. Arch Rheumatol 33, 498-504.

Gardosi, J., Kady, S.M., McGeown, P., Francis, A., and Tonks, A. (2005). Classification of stillbirth by relevant condition at death (ReCoDe): population based cohort study. BMJ 331, 1113-1117.

Gellhaus, A., Schmidt, M., Dunk, C., Lye, S.J., and Winterhager, E. (2007). The circulating proangiogenic factors CYR61 $(\mathrm{CCN} 1)$ and NOV $(\mathrm{CCN} 3)$ are significantly decreased in placentae and sera of preeclamptic patients. Reprod Sci 14, 46-52. 
Gilkes, D.M., Bajpai, S., Chaturvedi, P., Wirtz, D., and Semenza, G.L. (2013). Hypoxiainducible factor 1 (HIF-1) promotes extracellular matrix remodeling under hypoxic conditions by inducing P4HA1, P4HA2, and PLOD2 expression in fibroblasts. J Biol Chem 288, 10819-10829.

Gordijn, S.J., Beune, I.M., Thilaganathan, B., Papageorghiou, A., Baschat, A.A., Baker, P.N., Silver, R.M., Wynia, K., and Ganzevoort, W. (2016). Consensus definition of fetal growth restriction: a Delphi procedure. Ultrasound Obstet Gynecol 48, 333-339.

Hanna, J., Goldman-Wohl, D., Hamani, Y., Avraham, I., Greenfield, C., Natanson-Yaron, S., Prus, D., Cohen-Daniel, L., Arnon, T.I., Manaster, I., et al. (2006). Decidual NK cells regulate key developmental processes at the human fetal-maternal interface. Nat Med 12, 1065-1074.

Heinke, J., Wehofsits, L., Zhou, Q., Zoeller, C., Baar, K.M., Helbing, T., Laib, A., Augustin, H., Bode, C., Patterson, C., et al. (2008). BMPER is an endothelial cell regulator and controls bone morphogenetic protein-4-dependent angiogenesis. Circ Res 103, 804-812.

Hiby, S.E., Apps, R., Chazara, O., Farrell, L.E., Magnus, P., Trogstad, L., Gjessing, H.K., Carrington, M., and Moffett, A. (2014). Maternal KIR in combination with paternal HLA-C2 regulate human birth weight. J Immunol 192, 5069-5073.

Hiby, S.E., Apps, R., Sharkey, A.M., Farrell, L.E., Gardner, L., Mulder, A., Claas, F.H., Walker, J.J., Redman, C.W., Morgan, L., et al. (2010). Maternal activating KIRs protect against human reproductive failure mediated by fetal HLA-C2. J Clin Invest 120, 4102-4110. Hiby, S.E., Walker, J.J., O'Shaughnessy K, M., Redman, C.W., Carrington, M., Trowsdale, J., and Moffett, A. (2004). Combinations of maternal KIR and fetal HLA-C genes influence the risk of preeclampsia and reproductive success. J Exp Med 200, 957-965.

Hitz, C., Wurst, W., and Kuhn, R. (2007). Conditional brain-specific knockdown of MAPK using Cre/loxP regulated RNA interference. Nucleic Acids Res 35, e90.

Horikoshi, M., Beaumont, R.N., Day, F.R., Warrington, N.M., Kooijman, M.N., FernandezTajes, J., Feenstra, B., van Zuydam, N.R., Gaulton, K.J., Grarup, N., et al. (2016). Genomewide associations for birth weight and correlations with adult disease. Nature 538, 248-252.

http://www.informatics.jax.org/downloads/reports/HMD_HumanPhenotype.rpt.

http://www.informatics.jax.org/downloads/reports/HMD_HumanPhenotype.rpt.

Imdad, A., and Bhutta, Z.A. (2013). Nutritional management of the low birth weight/preterm infant in community settings: a perspective from the developing world. J Pediatr 162, S107114.

Jain, R., and Gray, D.H.D. (2014). Isolation of thymic epithelial cells and analysis by flow cytometry. Curr Protoc Immunol 107, 326 21-23 2615.

Januar, V., Desoye, G., Novakovic, B., Cvitic, S., and Saffery, R. (2015). Epigenetic regulation of human placental function and pregnancy outcome: considerations for causal inference. Am J Obstet Gynecol 213, S182-196.

Jin, R., De Smaele, E., Zazzeroni, F., Nguyen, D.U., Papa, S., Jones, J., Cox, C., Gelinas, C., and Franzoso, G. (2002). Regulation of the gadd45beta promoter by NF-kappaB. DNA Cell Biol 21, 491-503.

Johansson, S., Johansson, M., Rosmaraki, E., Vahlne, G., Mehr, R., Salmon-Divon, M., Lemonnier, F., Karre, K., and Hoglund, P. (2005). Natural killer cell education in mice with single or multiple major histocompatibility complex class I molecules. J Exp Med 201, 1145 1155 .

Juliano, P.B., Blotta, M.H., and Altemani, A.M. (2006). ICAM-1 is overexpressed by villous trophoblasts in placentitis. Placenta 27, 750-757.

Kaitu'u-Lino, T.J., Hastie, R., Cannon, P., Nguyen, H., Lee, S., Hannan, N.J., and Tong, S. (2015). Transcription factors E2F1 and E2F3 are expressed in placenta but do not regulate MMP14. Placenta 36, 932-937. 
Kamphaus, G.D., Colorado, P.C., Panka, D.J., Hopfer, H., Ramchandran, R., Torre, A., Maeshima, Y., Mier, J.W., Sukhatme, V.P., and Kalluri, R. (2000). Canstatin, a novel matrixderived inhibitor of angiogenesis and tumor growth. J Biol Chem 275, 1209-1215.

Karre, K., Ljunggren, H.G., Piontek, G., and Kiessling, R. (1986). Selective rejection of H-2deficient lymphoma variants suggests alternative immune defence strategy. Nature 319, 675678.

Kaur, G., Gras, S., Mobbs, J.I., Vivian, J.P., Cortes, A., Barber, T., Kuttikkatte, S.B., Jensen, L.T., Attfield, K.E., Dendrou, C.A., et al. (2017). Structural and regulatory diversity shape HLA-C protein expression levels. Nat Commun 8, 15924.

King, A., Burrows, T.D., Hiby, S.E., Bowen, J.M., Joseph, S., Verma, S., Lim, P.B., Gardner, L., Le Bouteiller, P., Ziegler, A., et al. (2000). Surface expression of HLA-C antigen by human extravillous trophoblast. Placenta 21, 376-387.

Kobayashi, H., Matsuda, Y., Hitomi, T., Okuda, H., Shioi, H., Matsuda, T., Imai, H., Sone, M., Taura, D., Harada, K.H., et al. (2015). Biochemical and Functional Characterization of RNF213 (Mysterin) R4810K, a Susceptibility Mutation of Moyamoya Disease, in Angiogenesis In Vitro and In Vivo. J Am Heart Assoc 4.

Kodym, R., Calkins, P., and Story, M. (1999). The cloning and characterization of a new stress response protein. A mammalian member of a family of theta class glutathione stransferase-like proteins. J Biol Chem 274, 5131-5137.

Kuleshov, M.V., Jones, M.R., Rouillard, A.D., Fernandez, N.F., Duan, Q., Wang, Z., Koplev, S., Jenkins, S.L., Jagodnik, K.M., Lachmann, A., et al. (2016). Enrichr: a comprehensive gene set enrichment analysis web server 2016 update. Nucleic Acids Res 44, W90-97. Lanier, L.L. (2005). NK cell recognition. Annu Rev Immunol 23, 225-274.

Lee, J., Beighley, P., Ritman, E., and Smith, N. (2007). Automatic segmentation of 3D micro-CT coronary vascular images. Med Image Anal 11, 630-647.

Lee, T.C., Kashyap, R.L., and Chu, C.N. (1994). Building Skeleton Models Via 3-D Medial Surface Axis Thinning Algorithms. Cvgip-Graph Model Im 56, 462-478.

Leon, D.A., Lithell, H.O., Vagero, D., Koupilova, I., Mohsen, R., Berglund, L., Lithell, U.B., and McKeigue, P.M. (1998). Reduced fetal growth rate and increased risk of death from ischaemic heart disease: cohort study of 15000 Swedish men and women born 1915-29. BMJ 317, 241-245.

Levine, R.J., Maynard, S.E., Qian, C., Lim, K.H., England, L.J., Yu, K.F., Schisterman, E.F., Thadhani, R., Sachs, B.P., Epstein, F.H., et al. (2004). Circulating angiogenic factors and the risk of preeclampsia. N Engl J Med 350, 672-683.

Li, Y., Lorca, R.A., and Su, E.J. (2018). Molecular and cellular underpinnings of normal and abnormal human placental blood flows. J Mol Endocrinol 60, R9-R22.

Li, Y., Zhang, J., Zhang, D., Hong, X., Tao, Y., Wang, S., Xu, Y., Piao, H., Yin, W., Yu, M., et al. (2017). Tim-3 signaling in peripheral NK cells promotes maternal-fetal immune

tolerance and alleviates pregnancy loss. Sci Signal 10.

Li, Y.H., Zhou, W.H., Tao, Y., Wang, S.C., Jiang, Y.L., Zhang, D., Piao, H.L., Fu, Q., Li, D.J., and Du, M.R. (2016). The Galectin-9/Tim-3 pathway is involved in the regulation of NK cell function at the maternal-fetal interface in early pregnancy. Cell Mol Immunol 13, 7381.

Liebermann, D.A., Tront, J.S., Sha, X., Mukherjee, K., Mohamed-Hadley, A., and Hoffman, B. (2011). Gadd45 stress sensors in malignancy and leukemia. Crit Rev Oncog 16, 129-140. Lima, P.D., Croy, B.A., Degaki, K.Y., Tayade, C., and Yamada, A.T. (2012). Heterogeneity in composition of mouse uterine natural killer cell granules. J Leukoc Biol 92, 195-204. Lokman, N.A., Ween, M.P., Oehler, M.K., and Ricciardelli, C. (2011). The role of annexin A2 in tumorigenesis and cancer progression. Cancer Microenviron 4, 199-208. 
Love, M.I., Huber, W., and Anders, S. (2014). Moderated estimation of fold change and dispersion for RNA-seq data with DESeq2. Genome Biol 15, 550.

Lu, H., Feng, Y., Hu, Y., Guo, Y., Liu, Y., Mao, Q., and Xue, W. (2020). Spondin 2

promotes the proliferation, migration and invasion of gastric cancer cells. J Cell Mol Med 24, 98-113.

Ma, D., Chang, L.Y., Zhao, S., Zhao, J.J., Xiong, Y.J., Cao, F.Y., Yuan, L., Zhang, Q., Wang, X.Y., Geng, M.L., et al. (2017). KLF5 promotes cervical cancer proliferation, migration and invasion in a manner partly dependent on TNFRSF11a expression. Sci Rep 7, 15683.

Malhotra, A., Allison, B.J., Castillo-Melendez, M., Jenkin, G., Polglase, G.R., and Miller, S.L. (2019). Neonatal Morbidities of Fetal Growth Restriction: Pathophysiology and Impact. Front Endocrinol (Lausanne) 10, 55.

Marusina, A.I., Burgess, S.J., Pathmanathan, I., Borrego, F., and Coligan, J.E. (2008). Regulation of human DAP10 gene expression in NK and T cells by Ap-1 transcription factors. J Immunol 180, 409-417.

McCowan, L.M., Figueras, F., and Anderson, N.H. (2018). Evidence-based national guidelines for the management of suspected fetal growth restriction: comparison, consensus, and controversy. Am J Obstet Gynecol 218, S855-S868.

McInnes, L., Healy, J., and Melville, J. (2018). UMAP: Uniform Manifold Approximation and Projection for dimension reduction. Preprint at https://arxivorg/abs/180203426.

Meng, Q., Mongan, M., Wang, J., and Xia, Y. (2018). Repression of MAP3K1 expression and JNK activity by canonical Wnt signaling. Dev Biol 440, 129-136.

Moffett, A., and Loke, C. (2006). Immunology of placentation in eutherian mammals. Nat Rev Immunol 6, 584-594.

Moraitis, A.A., Wood, A.M., Fleming, M., and Smith, G.C. (2014). Birth weight percentile and the risk of term perinatal death. Obstet Gynecol 124, 274-283.

Mukherjee, A.B., Zhang, Z., and Chilton, B.S. (2007). Uteroglobin: a steroid-inducible immunomodulatory protein that founded the Secretoglobin superfamily. Endocr Rev 28, $707-$ 725.

Murthi, P., van Zanten, D.E., Eijsink, J.J., Borg, A.J., Stevenson, J.L., Kalionis, B., Chui, A.K., Said, J.M., Brennecke, S.P., and Erwich, J.J. (2016). Decorin expression is decreased in first trimester placental tissue from pregnancies with small for gestation age infants at birth. Placenta 45, 58-62.

Nakimuli, A., Chazara, O., Hiby, S.E., Farrell, L., Tukwasibwe, S., Jayaraman, J., Traherne, J.A., Trowsdale, J., Colucci, F., Lougee, E., et al. (2015). A KIR B centromeric region present in Africans but not Europeans protects pregnant women from pre-eclampsia. Proc Natl Acad Sci U S A 112, 845-850.

Nardozza, L.M., Caetano, A.C., Zamarian, A.C., Mazzola, J.B., Silva, C.P., Marcal, V.M., Lobo, T.F., Peixoto, A.B., and Araujo Junior, E. (2017). Fetal growth restriction: current knowledge. Arch Gynecol Obstet 295, 1061-1077.

Nawathe, A.R., Christian, M., Kim, S.H., Johnson, M., Savvidou, M.D., and Terzidou, V. (2016). Insulin-like growth factor axis in pregnancies affected by fetal growth disorders. Clin Epigenetics 8, 11.

Ndhlovu, L.C., Lopez-Verges, S., Barbour, J.D., Jones, R.B., Jha, A.R., Long, B.R., Schoeffler, E.C., Fujita, T., Nixon, D.F., and Lanier, L.L. (2012). Tim-3 marks human natural killer cell maturation and suppresses cell-mediated cytotoxicity. Blood 119, 3734-3743. Nelson, A.C., Mould, A.W., Bikoff, E.K., and Robertson, E.J. (2016). Single-cell RNA-seq reveals cell type-specific transcriptional signatures at the maternal-foetal interface during pregnancy. Nat Commun 7, 11414. 
Oberg, L., Johansson, S., Michaelsson, J., Tomasello, E., Vivier, E., Karre, K., and Hoglund, P. (2004). Loss or mismatch of MHC class I is sufficient to trigger NK cell-mediated rejection of resting lymphocytes in vivo - role of KARAP/DAP12-dependent and independent pathways. Eur J Immunol 34, 1646-1653.

Ohkubo, K., Sakai, Y., Inoue, H., Akamine, S., Ishizaki, Y., Matsushita, Y., Sanefuji, M., Torisu, H., Ihara, K., Sardiello, M., et al. (2015). Moyamoya disease susceptibility gene RNF213 links inflammatory and angiogenic signals in endothelial cells. Sci Rep 5, 13191. Okada, S., Okada, H., Sanezumi, M., Nakajima, T., Yasuda, K., and Kanzaki, H. (2000). Expression of interleukin-15 in human endometrium and decidua. Mol Hum Reprod 6, 75-80. Orsolic, I., Jurada, D., Pullen, N., Oren, M., Eliopoulos, A.G., and Volarevic, S. (2016). The relationship between the nucleolus and cancer: Current evidence and emerging paradigms. Semin Cancer Biol 37-38, 36-50.

Otsuka, A., Hanafusa, T., Kono, N., and Tarui, S. (1991). Lipopolysaccharide augments HLA-A,B,C molecule expression but inhibits interferon-gamma-induced HLA-DR molecule expression on cultured human endothelial cells. Immunology 73, 428-432.

Paffaro, V.A., Jr., Bizinotto, M.C., Joazeiro, P.P., and Yamada, A.T. (2003). Subset classification of mouse uterine natural killer cells by DBA lectin reactivity. Placenta 24, 479488.

Pang, S.C., Janzen-Pang, J., Tse, M.Y., Croy, B.A., and Tse, D. (2014). 2 - Implant Site Dissections. In The Guide to Investigation of Mouse Pregnancy, B.A. Croy, A.T. Yamada, F.J. DeMayo, and S.L. Adamson, eds. (Boston: Academic Press), pp. 21-42.

Parham, P. (2005). MHC class I molecules and KIRs in human history, health and survival. Nat Rev Immunol 5, 201-214.

Pavlicev, M., Wagner, G.P., Chavan, A.R., Owens, K., Maziarz, J., Dunn-Fletcher, C., Kallapur, S.G., Muglia, L., and Jones, H. (2017). Single-cell transcriptomics of the human placenta: inferring the cell communication network of the maternal-fetal interface. Genome Res 27, 349-361.

Perschbacher, K.J., Deng, G., Sandgren, J.A., Walsh, J.W., Witcher, P.C., Sapouckey, S.A., Owens, C.E., Zhang, S.Y., Scroggins, S.M., Pearson, N.A., et al. (2020). Reduced mRNA Expression of RGS2 (Regulator of G Protein Signaling-2) in the Placenta Is Associated With Human Preeclampsia and Sufficient to Cause Features of the Disorder in Mice. Hypertension 75, 569-579.

Picelli, S., Faridani, O.R., Bjorklund, A.K., Winberg, G., Sagasser, S., and Sandberg, R. (2014). Full-length RNA-seq from single cells using Smart-seq2. Nat Protoc 9, 171-181. Polytarchou, C., Hatziapostolou, M., Yau, T.O., Christodoulou, N., Hinds, P.W., Kottakis, F., Sanidas, I., and Tsichlis, P.N. (2020). Akt3 induces oxidative stress and DNA damage by activating the NADPH oxidase via phosphorylation of p47(phox). Proc Natl Acad Sci U S A 117, 28806-28815.

Rabinowich, H., Pricop, L., Herberman, R.B., and Whiteside, T.L. (1994). Expression and function of CD7 molecule on human natural killer cells. J Immunol 152, 517-526.

Ramathal, C.Y., Bagchi, I.C., Taylor, R.N., and Bagchi, M.K. (2010). Endometrial decidualization: of mice and men. Semin Reprod Med 28, 17-26.

Raymond, C.S., and Soriano, P. (2007). High-efficiency FLP and PhiC31 site-specific recombination in mammalian cells. PLoS One 2, e162.

Rennie, M.Y., Whiteley, K.J., Sled, J.G., and Adamson, S.L. (2014). 55 - Scanning Electron Microscopy and Micro-Computed Tomography Imaging of the Utero- and Fetoplacental Circulations. In The Guide to Investigation of Mouse Pregnancy, B.A. Croy, A.T. Yamada, F.J. DeMayo, and S.L. Adamson, eds. (Boston: Academic Press), pp. 637-648.

Resnik, R. (2002). Intrauterine growth restriction. Obstet Gynecol 99, 490-496. 
Rich-Edwards, J.W., Stampfer, M.J., Manson, J.E., Rosner, B., Hankinson, S.E., Colditz, G.A., Willett, W.C., and Hennekens, C.H. (1997). Birth weight and risk of cardiovascular disease in a cohort of women followed up since 1976. BMJ 315, 396-400.

Robillard, P.Y., Dekker, G.A., and Hulsey, T.C. (2002). Evolutionary adaptations to preeclampsia/eclampsia in humans: low fecundability rate, loss of oestrus, prohibitions of incest and systematic polyandry. Am J Reprod Immunol 47, 104-111.

Rodrigues-Duarte, L., Pandya, Y., Neres, R., and Penha-Goncalves, C. (2018). Fetal and Maternal Innate Immunity Receptors Have Opposing Effects on the Severity of Experimental Malaria in Pregnancy: Beneficial Roles for Fetus-Derived Toll-Like Receptor 4 and Type I Interferon Receptor 1. Infect Immun 86.

Rodriguez, A., Ehlenberger, D.B., Hof, P.R., and Wearne, S.L. (2006). Rayburst sampling, an algorithm for automated three-dimensional shape analysis from laser scanning microscopy images. Nat Protoc 1, 2152-2161.

Romo, A., Carceller, R., and Tobajas, J. (2009). Intrauterine growth retardation (IUGR): epidemiology and etiology. Pediatr Endocrinol Rev 6 Suppl 3, 332-336.

Rosenberg, A. (2008). The IUGR newborn. Semin Perinatol 32, 219-224.

Satoyoshi, R., Kuriyama, S., Aiba, N., Yashiro, M., and Tanaka, M. (2015). Asporin activates coordinated invasion of scirrhous gastric cancer and cancer-associated fibroblasts. Oncogene 34, 650-660.

Scholz, B., Korn, C., Wojtarowicz, J., Mogler, C., Augustin, I., Boutros, M., Niehrs, C., and Augustin, H.G. (2016). Endothelial RSPO3 Controls Vascular Stability and Pruning through Non-canonical WNT/Ca(2+)/NFAT Signaling. Dev Cell 36, 79-93.

Shannon, P., Markiel, A., Ozier, O., Baliga, N.S., Wang, J.T., Ramage, D., Amin, N., Schwikowski, B., and Ideker, T. (2003). Cytoscape: a software environment for integrated models of biomolecular interaction networks. Genome Res 13, 2498-2504.

Shindo, T., Manabe, I., Fukushima, Y., Tobe, K., Aizawa, K., Miyamoto, S., Kawai-Kowase, K., Moriyama, N., Imai, Y., Kawakami, H., et al. (2002). Kruppel-like zinc-finger transcription factor KLF5/BTEB2 is a target for angiotensin II signaling and an essential regulator of cardiovascular remodeling. Nat Med 8, 856-863.

Smillie, C.S., Biton, M., Ordovas-Montanes, J., Sullivan, K.M., Burgin, G., Graham, D.B., Herbst, R.H., Rogel, N., Slyper, M., Waldman, J., et al. (2019). Intra- and Inter-cellular Rewiring of the Human Colon during Ulcerative Colitis. Cell 178, 714-730 e 722.

Sojka, D.K., Plougastel-Douglas, B., Yang, L., Pak-Wittel, M.A., Artyomov, M.N., Ivanova, Y., Zhong, C., Chase, J.M., Rothman, P.B., Yu, J., et al. (2014). Tissue-resident natural killer (NK) cells are cell lineages distinct from thymic and conventional splenic NK cells. Elife 3, e01659.

Sojka, D.K., Yang, L., Plougastel-Douglas, B., Higuchi, D.A., Croy, B.A., and Yokoyama, W.M. (2018). Cutting Edge: Local Proliferation of Uterine Tissue-Resident NK Cells during Decidualization in Mice. J Immunol 201, 2551-2556.

Steegers, E.A., von Dadelszen, P., Duvekot, J.J., and Pijnenborg, R. (2010). Pre-eclampsia. Lancet 376, 631-644.

Stefanoska, I., Jovanovic Krivokuca, M., Vasilijic, S., Cujic, D., and Vicovac, L. (2013). Prolactin stimulates cell migration and invasion by human trophoblast in vitro. Placenta 34 , 775-783.

Strimmer, K. (2008). fdrtool: a versatile R package for estimating local and tail area-based false discovery rates. Bioinformatics 24, 1461-1462.

Subramanian, A., Vernon, K.A., Slyper, M., Waldman, J., Luecken, M.D., Gosik, K., Dubinsky, D., Cuoco, M.S., Keller, K., Purnell, J., et al. (2020). RAAS blockade, kidney disease, and expression of $<\mathrm{em}>\mathrm{ACE} 2</ \mathrm{em}>$, the entry receptor for SARS-CoV-2, in kidney epithelial and endothelial cells. bioRxiv, 2020.2006.2023.167098. 
Subramanian Vignesh, K., and Deepe, G.S., Jr. (2017). Metallothioneins: Emerging Modulators in Immunity and Infection. Int J Mol Sci 18.

Tirosh, I., Izar, B., Prakadan, S.M., Wadsworth, M.H., 2nd, Treacy, D., Trombetta, J.J., Rotem, A., Rodman, C., Lian, C., Murphy, G., et al. (2016). Dissecting the multicellular ecosystem of metastatic melanoma by single-cell RNA-seq. Science 352, 189-196.

Togher, K.L., Togher, K.L., O'Keeffe, M.M., O'Keeffe, M.M., Khashan, A.S., Khashan, A.S., Gutierrez, H., Gutierrez, H., Kenny, L.C., Kenny, L.C., et al. (2014). Epigenetic regulation of the placental HSD11B2 barrier and its role as a critical regulator of fetal development.

Epigenetics 9, 816-822.

Tossetta, G., Fantone, S., Giannubilo, S.R., Marinelli Busilacchi, E., Ciavattini, A., Castellucci, M., Di Simone, N., Mattioli-Belmonte, M., and Marzioni, D. (2019). Preeclampsia onset and SPARC: A possible involvement in placenta development. J Cell Physiol 234, 6091-6098.

Trombetta, J.J., Gennert, D., Lu, D., Satija, R., Shalek, A.K., and Regev, A. (2014).

Preparation of Single-Cell RNA-Seq Libraries for Next Generation Sequencing. Curr Protoc Mol Biol 107, 422 21-17.

Trowsdale, J. (2001). Genetic and functional relationships between MHC and NK receptor genes. Immunity 15, 363-374.

Tsang, J.C.H., Vong, J.S.L., Ji, L., Poon, L.C.Y., Jiang, P., Lui, K.O., Ni, Y.B., To, K.F., Cheng, Y.K.Y., Chiu, R.W.K., et al. (2017). Integrative single-cell and cell-free plasma RNA transcriptomics elucidates placental cellular dynamics. Proc Natl Acad Sci U S A 114, E7786-E7795.

Tyrrell, J.S., Yaghootkar, H., Freathy, R.M., Hattersley, A.T., and Frayling, T.M. (2013). Parental diabetes and birthweight in 236030 individuals in the UK biobank study. Int J Epidemiol 42, 1714-1723.

Vento-Tormo, R., Efremova, M., Botting, R.A., Turco, M.Y., Vento-Tormo, M., Meyer, K.B., Park, J.E., Stephenson, E., Polanski, K., Goncalves, A., et al. (2018). Single-cell reconstruction of the early maternal-fetal interface in humans. Nature 563, 347-353. Vilches, C., and Parham, P. (2002). KIR: diverse, rapidly evolving receptors of innate and adaptive immunity. Annu Rev Immunol 20, 217-251.

Wallrapp, A., Riesenfeld, S.J., Burkett, P.R., Abdulnour, R.E., Nyman, J., Dionne, D., Hofree, M., Cuoco, M.S., Rodman, C., Farouq, D., et al. (2017). The neuropeptide NMU amplifies ILC2-driven allergic lung inflammation. Nature 549, 351-356.

Wang, G., Zhang, Z., Chen, C., Zhang, Y., and Zhang, C. (2016). Dysfunction of WNT4/WNT5A in deciduas: possible relevance to the pathogenesis of preeclampsia. J Hypertens 34, 719-727.

Warrington, N.M., Beaumont, R.N., Horikoshi, M., Day, F.R., Helgeland, O., Laurin, C., Bacelis, J., Peng, S., Hao, K., Feenstra, B., et al. (2019). Maternal and fetal genetic effects on birth weight and their relevance to cardio-metabolic risk factors. Nat Genet 51, 804-814. West, R.C., Bouma, G.J., and Winger, Q.A. (2018). Shifting perspectives from "oncogenic" to oncofetal proteins; how these factors drive placental development. Reprod Biol Endocrinol $16,101$.

Wie, J.H., Ko, H.S., Choi, S.K., Park, I.Y., Kim, A., Kim, H.S., and Shin, J.C. (2018). Effects of Oncostatin M on Invasion of Primary Trophoblasts under Normoxia and Hypoxia Conditions. Yonsei Med J 59, 879-886.

Wolock, S.L., Lopez, R., and Klein, A.M. (2019). Scrublet: Computational Identification of Cell Doublets in Single-Cell Transcriptomic Data. Cell Syst 8, 281-291 e289.

Xu, H., Ding, J., Porter, C.B.M., Wallrapp, A., Tabaka, M., Ma, S., Fu, S., Guo, X., Riesenfeld, S.J., Su, C., et al. (2019). Transcriptional Atlas of Intestinal Immune Cells 
Reveals that Neuropeptide alpha-CGRP Modulates Group 2 Innate Lymphoid Cell Responses. Immunity 51, 696-708 e699.

Yabe, S., Alexenko, A.P., Amita, M., Yang, Y., Schust, D.J., Sadovsky, Y., Ezashi, T., and Roberts, R.M. (2016). Comparison of syncytiotrophoblast generated from human embryonic stem cells and from term placentas. Proc Natl Acad Sci U S A 113, E2598-2607.

Yang, J., Anholts, J., Kolbe, U., Stegehuis-Kamp, J.A., Claas, F.H.J., and Eikmans, M. (2018). Calcium-Binding Proteins S100A8 and S100A9: Investigation of Their Immune Regulatory Effect in Myeloid Cells. Int J Mol Sci 19.

Ye, W., Zheng, L.M., Young, J.D., and Liu, C.C. (1996). The involvement of interleukin (IL)-15 in regulating the differentiation of granulated metrial gland cells in mouse pregnant uterus. J Exp Med 184, 2405-2410.

Zeisler, H., Llurba, E., Chantraine, F., Vatish, M., Staff, A.C., Sennstrom, M., Olovsson, M., Brennecke, S.P., Stepan, H., Allegranza, D., et al. (2016). Predictive Value of the sFlt-1:PlGF Ratio in Women with Suspected Preeclampsia. N Engl J Med 374, 13-22.

Zhang, Q., and Yan, J. (2016). Update of Wnt signaling in implantation and decidualization. Reprod Med Biol 15, 95-105.

Zhang, Y., Diao, Z., Su, L., Sun, H., Li, R., Cui, H., and Hu, Y. (2010). MicroRNA-155 contributes to preeclampsia by down-regulating CYR61. Am J Obstet Gynecol 202, 466 e461-467.

Zhang, Z., Zhang, L., Zhang, L., Jia, L., Wang, P., and Gao, Y. (2013). Association of Wnt2 and sFRP4 expression in the third trimester placenta in women with severe preeclampsia. Reprod Sci 20, 981-989.

Zhao, W.X., and Lin, J.H. (2012). Notch signaling pathway and human placenta. Int J Med Sci 9, 447-452.

Zuckerwise, L., Li, J., Lu, L., Men, Y., Geng, T., Buhimschi, C.S., Buhimschi, I.A., Bukowski, R., Guller, S., Paidas, M., et al. (2016). H19 long noncoding RNA alters trophoblast cell migration and invasion by regulating TbetaR 3 in placentae with fetal growth restriction. Oncotarget 7, 38398-38407. 

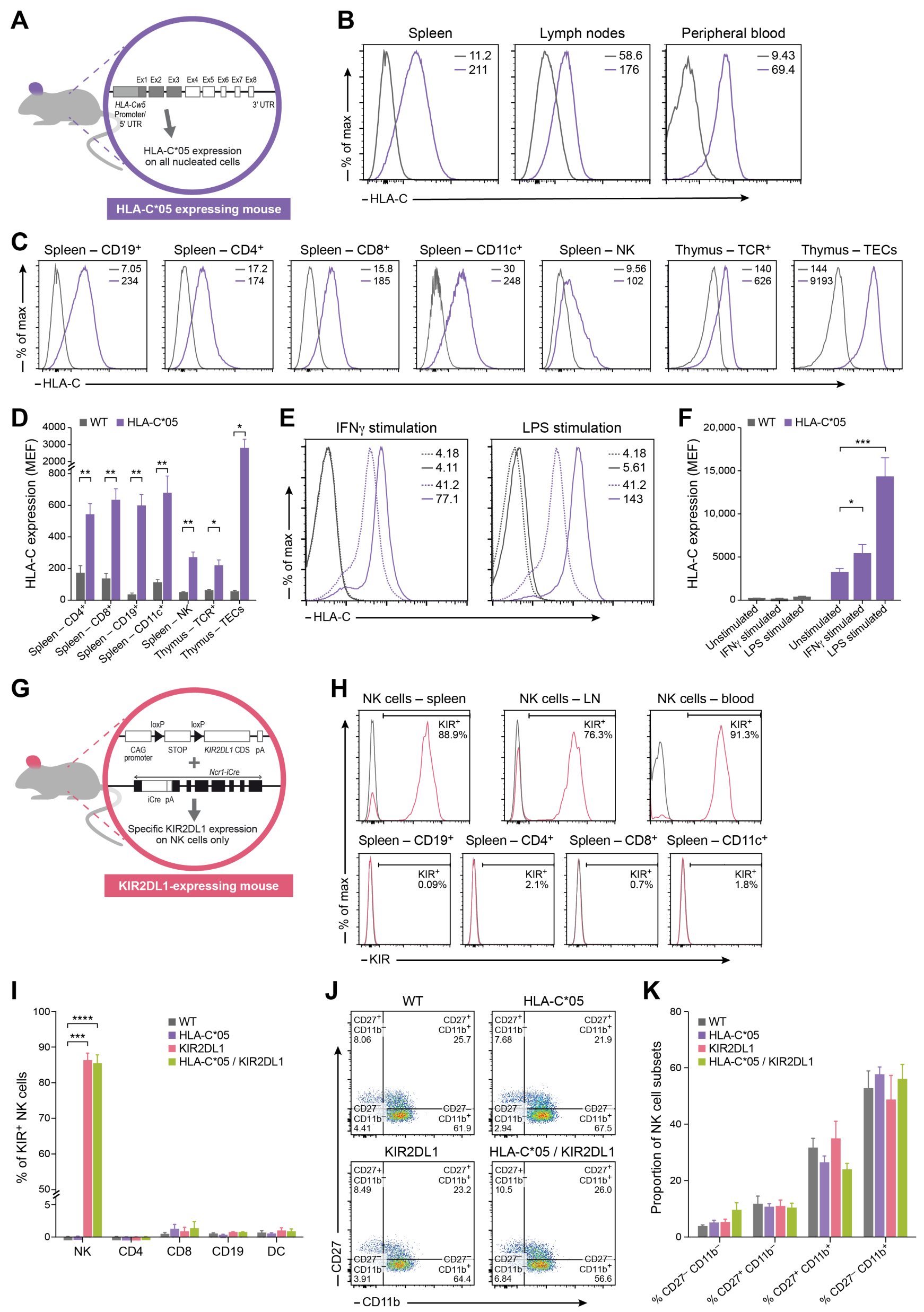
A

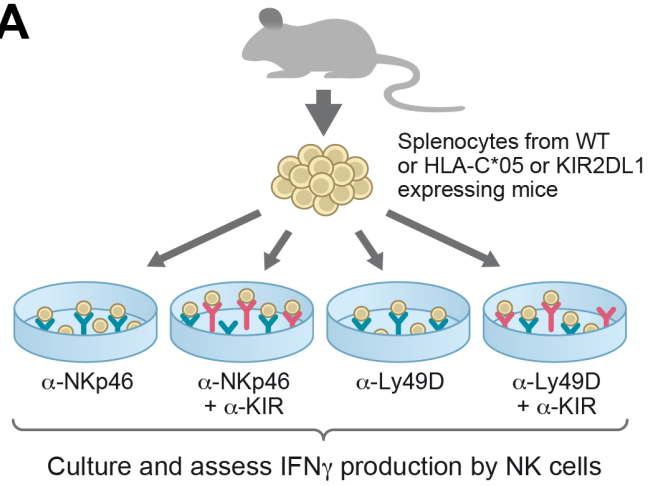

C

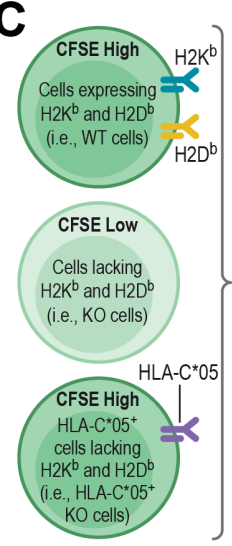

E

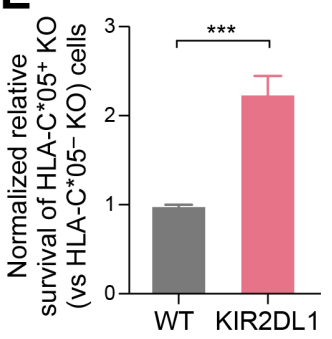

F $\quad \mathrm{KIR} \times \mathrm{C}^{*} 05$

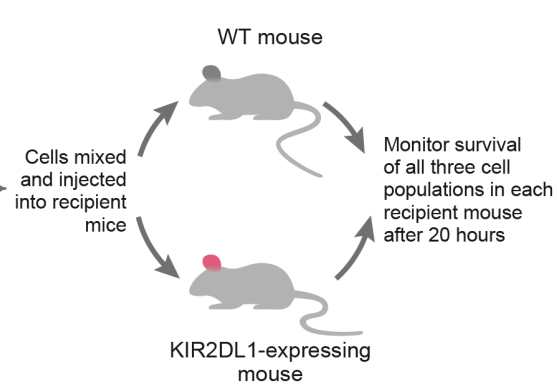

B

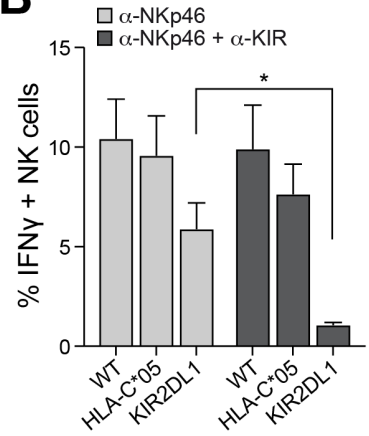

D Injection mix

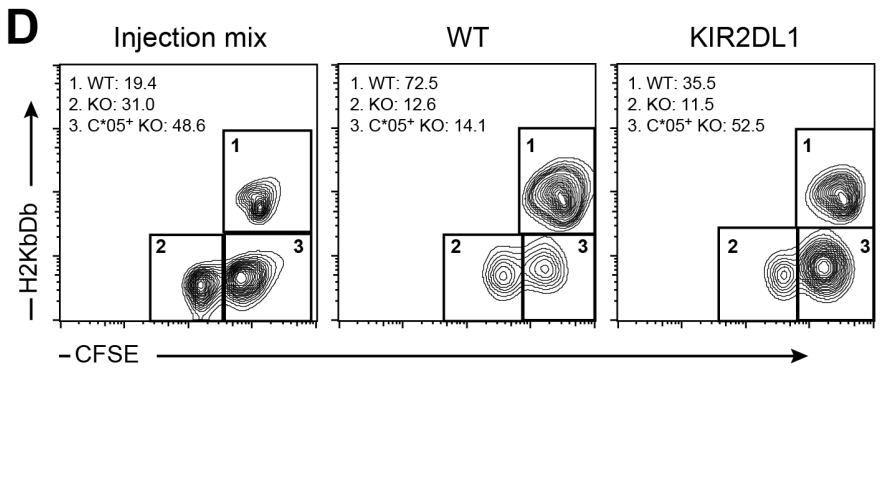

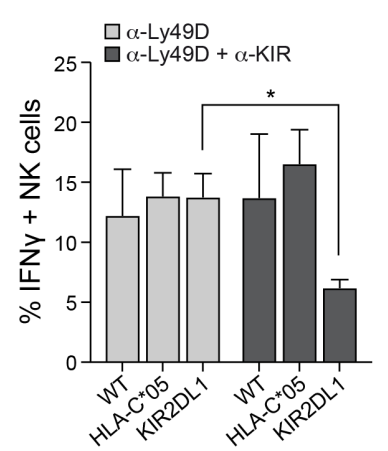

Figure 2
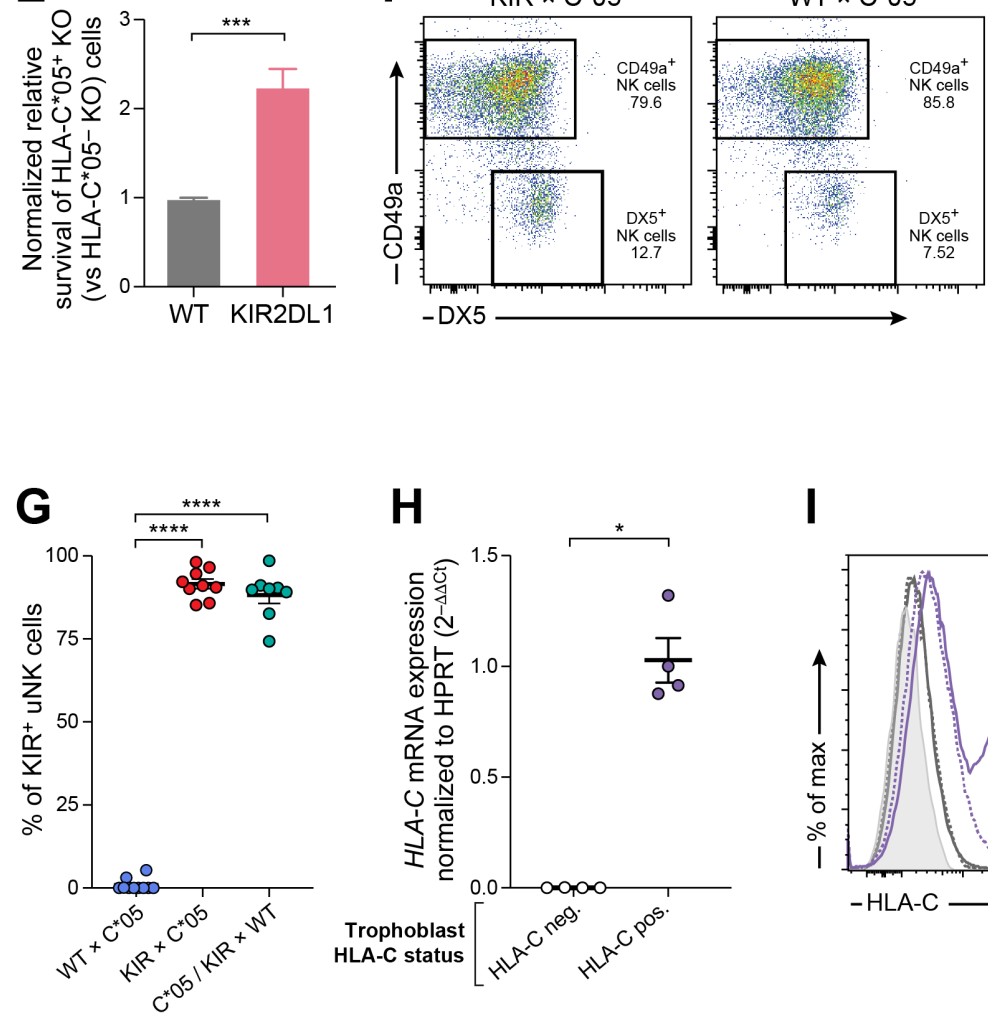

H

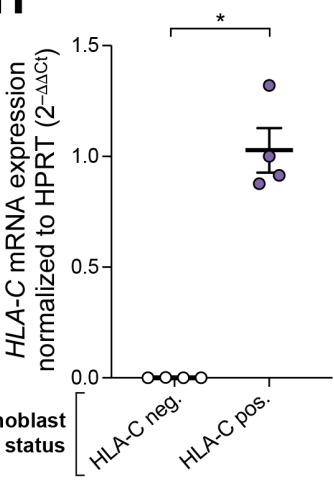

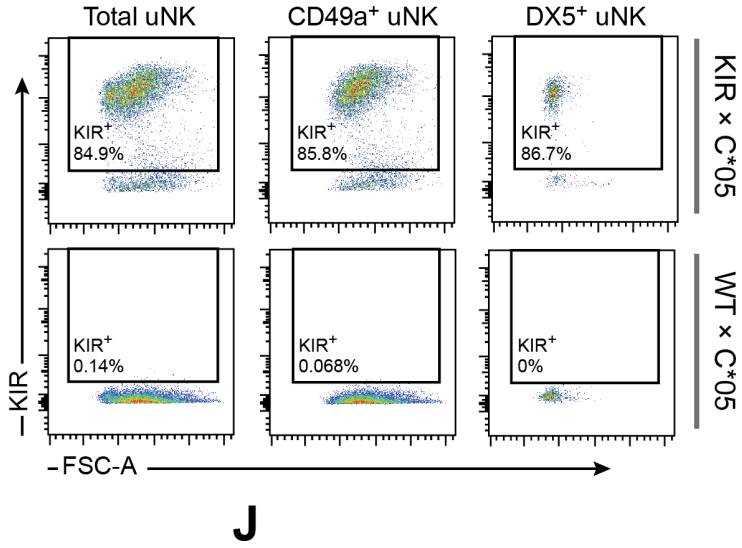
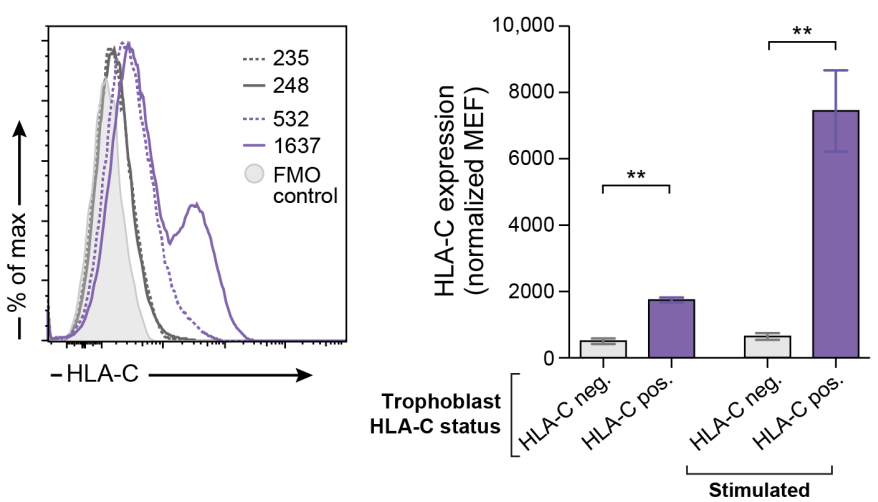
Figure 3

A

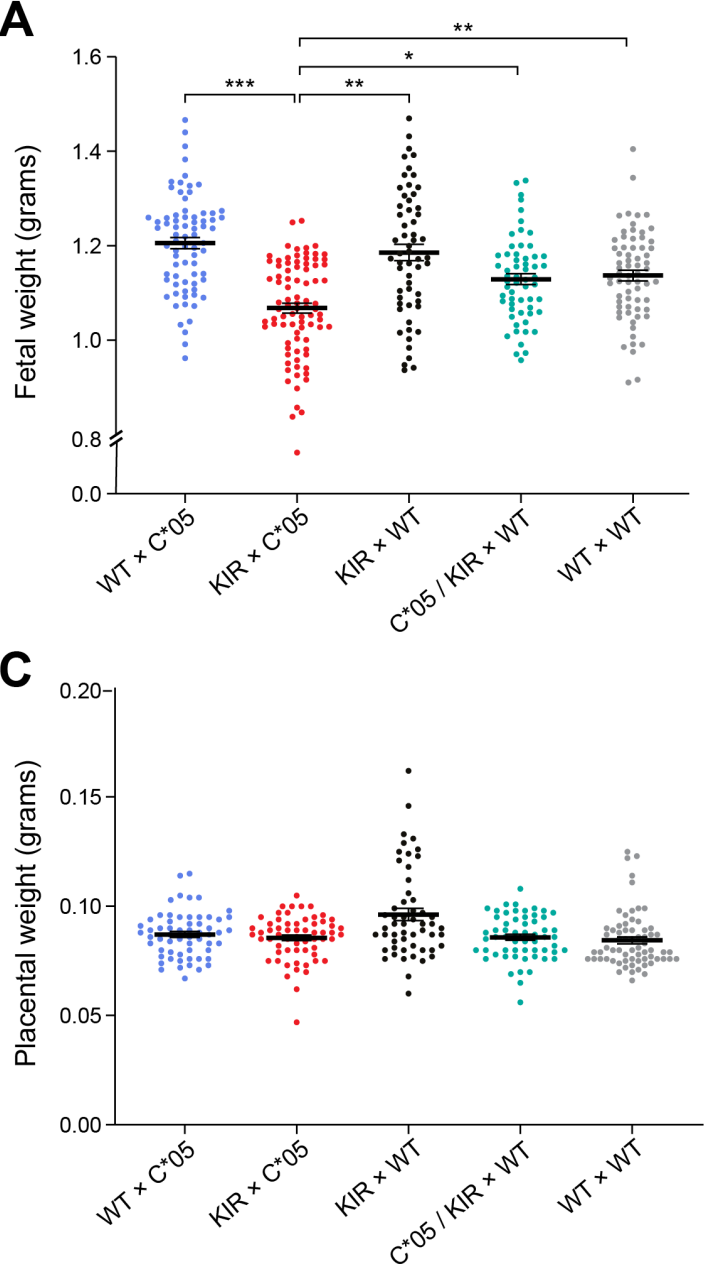

B

\begin{tabular}{|c|c|c|c|}
\hline $\begin{array}{c}\text { Mating } \\
\text { combination }\end{array}$ & $\begin{array}{c}\text { Female gene } \\
\text { expression }\end{array}$ & $\begin{array}{l}\text { Male gene } \\
\text { expression }\end{array}$ & $\begin{array}{l}\text { Fetal weight } \\
\text { proportions }\end{array}$ \\
\hline$W T \times C^{*} 05$ & $\begin{array}{c}\text { No KIR2DL1 } \\
\text { expression }\end{array}$ & $\begin{array}{l}\text { HLA-C*05 on } \\
\text { all nucleated } \\
\text { cells }\end{array}$ & $\begin{array}{l}-5.41 \% \\
-5.41 \% \\
-89.19 \%\end{array}$ \\
\hline $\mathrm{KIR} \times \mathrm{C}^{*} 05$ & $\begin{array}{l}\text { KIR2DL1 } \\
\text { expression } \\
\text { on NK cells }\end{array}$ & $\begin{array}{l}\text { HLA-C*05 on } \\
\text { all nucleated } \\
\text { cells }\end{array}$ & $\begin{array}{r}-36.05 \% \\
19.77 \%\end{array}$ \\
\hline $\mathrm{KIR} \times \mathrm{WT}$ & $\begin{array}{l}\text { KIR2DL1 } \\
\text { expression } \\
\text { on NK cells }\end{array}$ & $\begin{array}{l}\text { No HLA-C*05 } \\
\text { expression }\end{array}$ & $\begin{array}{l}15.52 \% \\
8.62 \% \\
75.86 \%\end{array}$ \\
\hline $\mathrm{C}^{*} 05 / \mathrm{KIR} \times \mathrm{WT}$ & $\begin{array}{l}\text { KIR2DL1 } \\
\text { expression on } \\
\text { NK cells and } \\
\text { HLA-C*05 on } \\
\text { all nucleated } \\
\text { cells }\end{array}$ & $\begin{array}{l}\text { No HLA-C*05 } \\
\text { expression }\end{array}$ & $\begin{array}{r}11.94 \% \\
14.93 \% \\
73.13 \%\end{array}$ \\
\hline $\mathrm{WT} \times \mathrm{WT}$ & $\begin{array}{l}\text { No KIR2DL1 } \\
\text { expression }\end{array}$ & $\begin{array}{l}\text { No HLA-C*05 } \\
\text { expression }\end{array}$ & $\begin{array}{l}=15.25 \% \\
13.56 \%\end{array}$ \\
\hline
\end{tabular}

- < 5th percentile of WT $\times C^{*} 05$ fetuses

5th-10th percentile of WT $\times C^{*} 05$ fetuses

- > 10th percentile of WT $\times C^{*} 05$ fetuses 
A

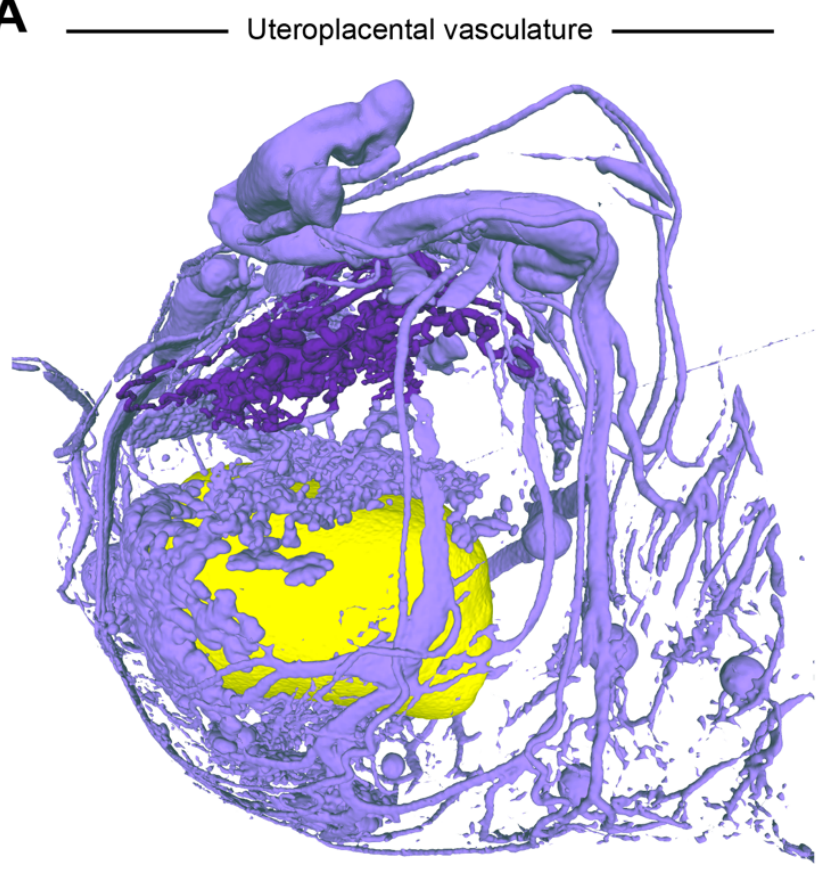

D

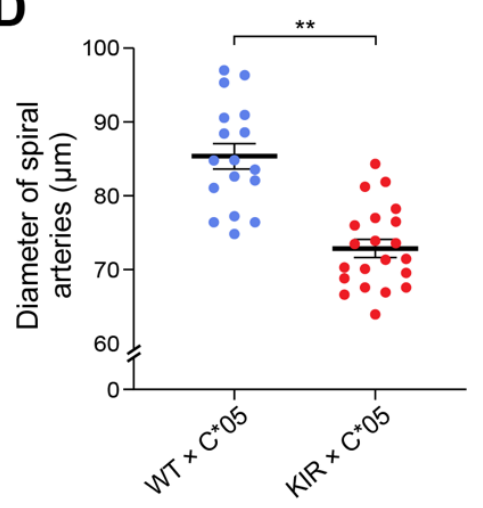

G

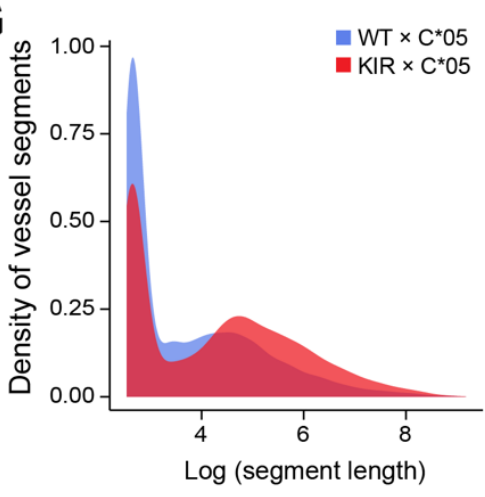

B

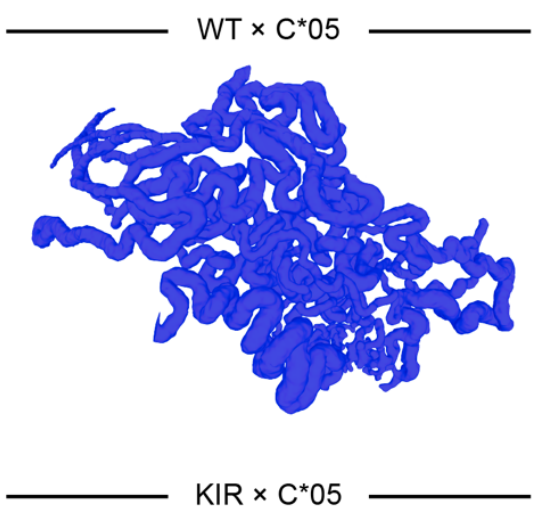

\section{C}
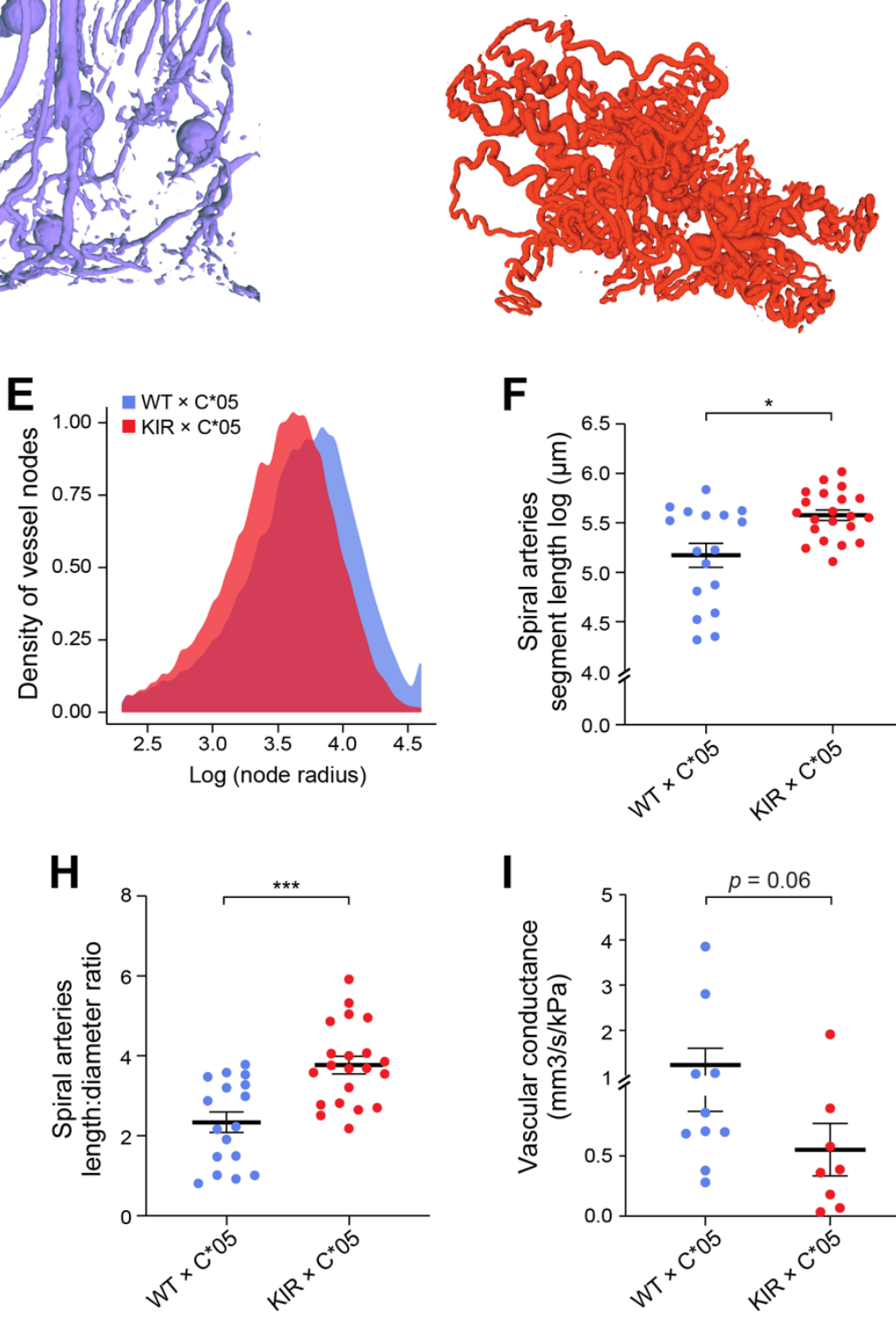

$\mathbf{F}$

Figure 4
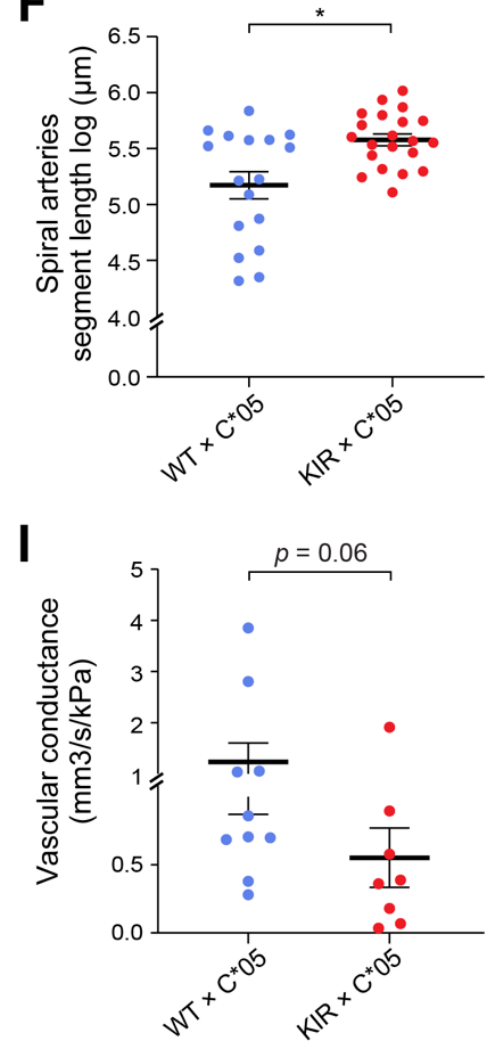
A

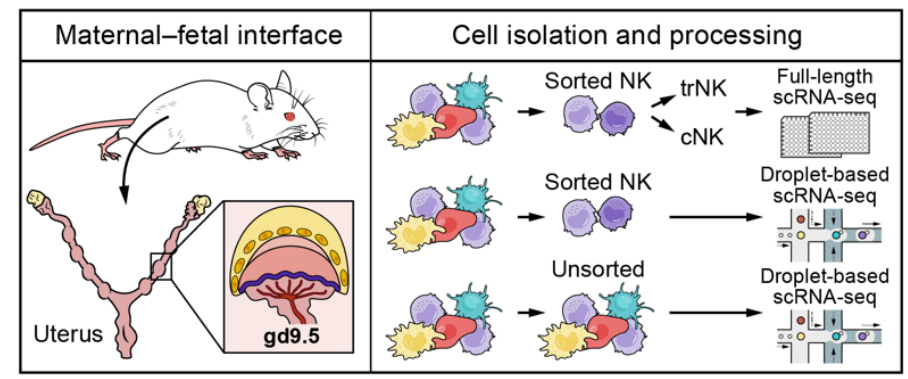

C

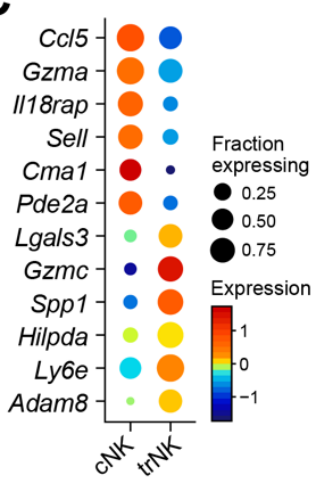

F

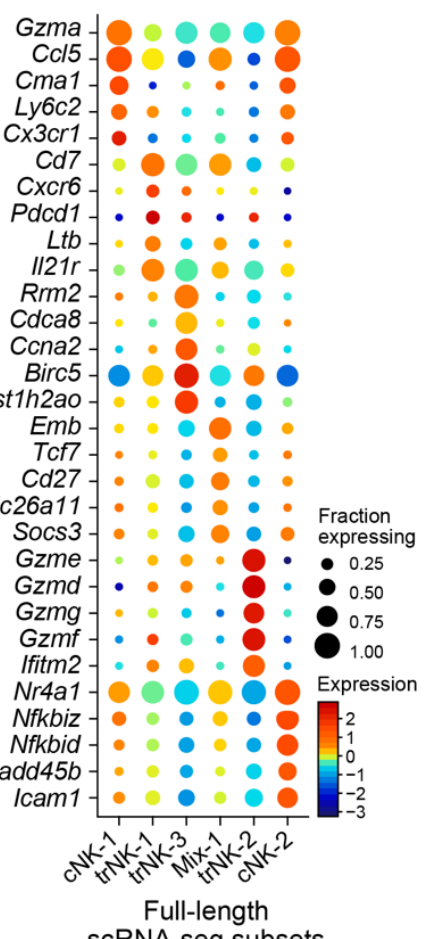

scRNA-seq subsets
D

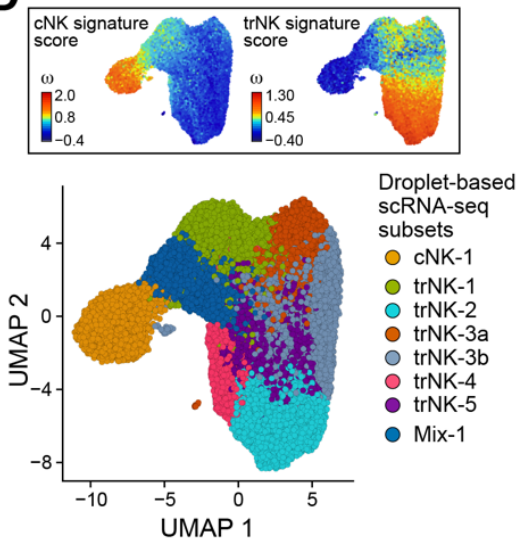

G

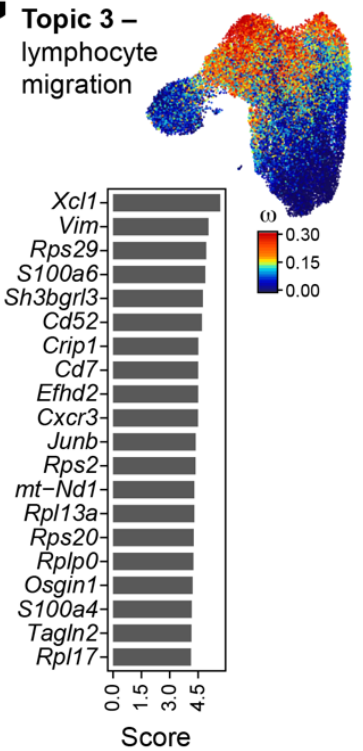

B

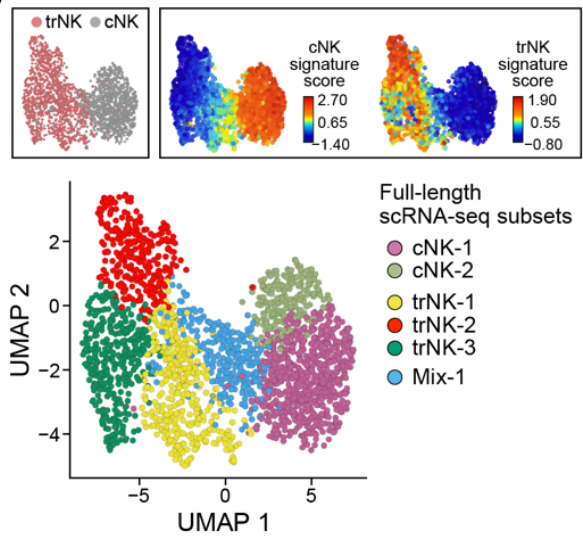

E

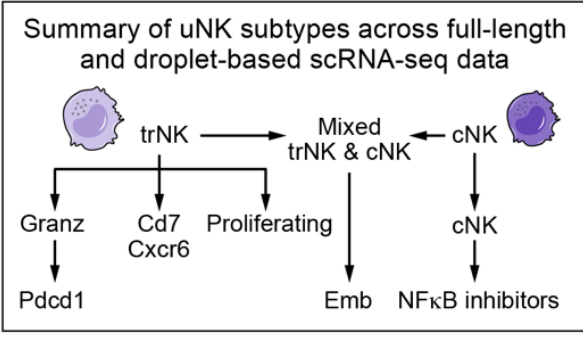

Figure 5

$\mathbf{H}_{\text {Topic } 8 \text { - }}$ transcriptional regulation splicing

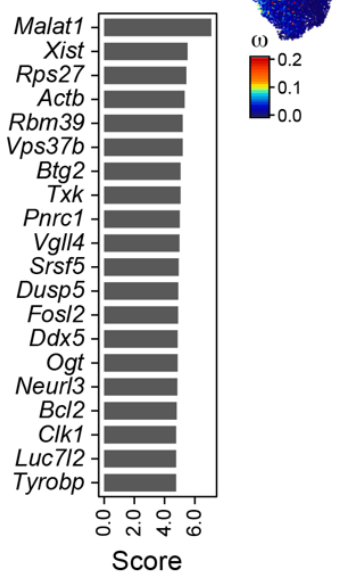

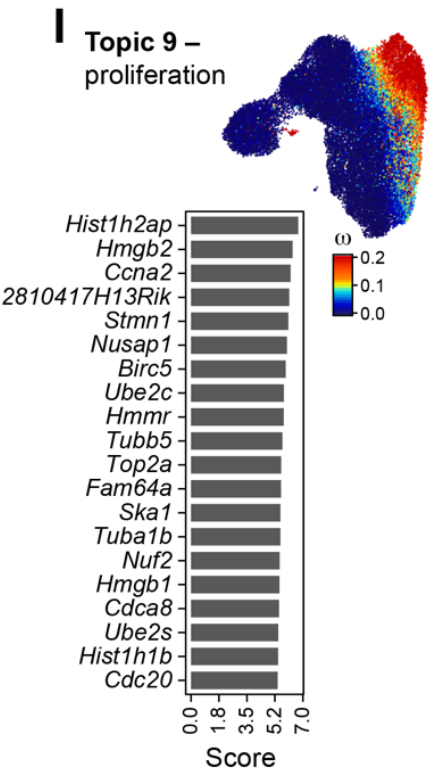


A

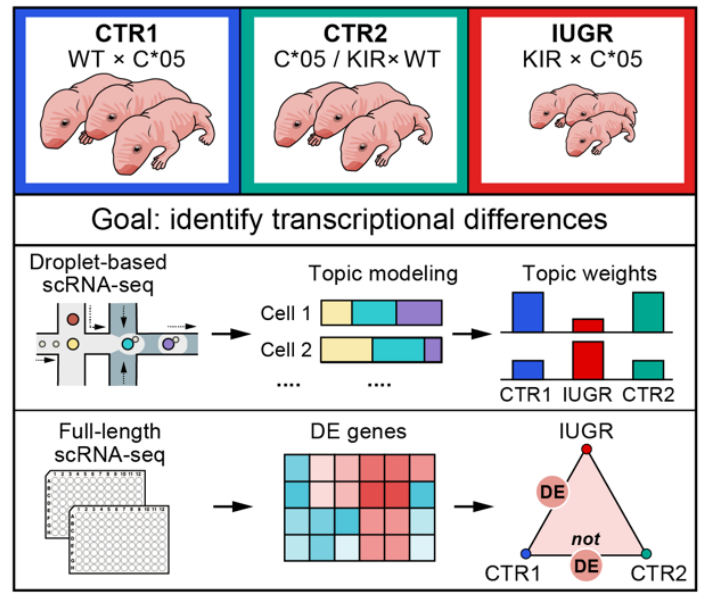

B

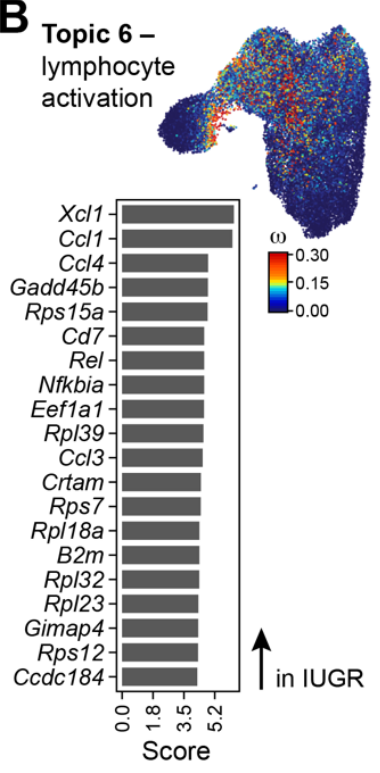

E
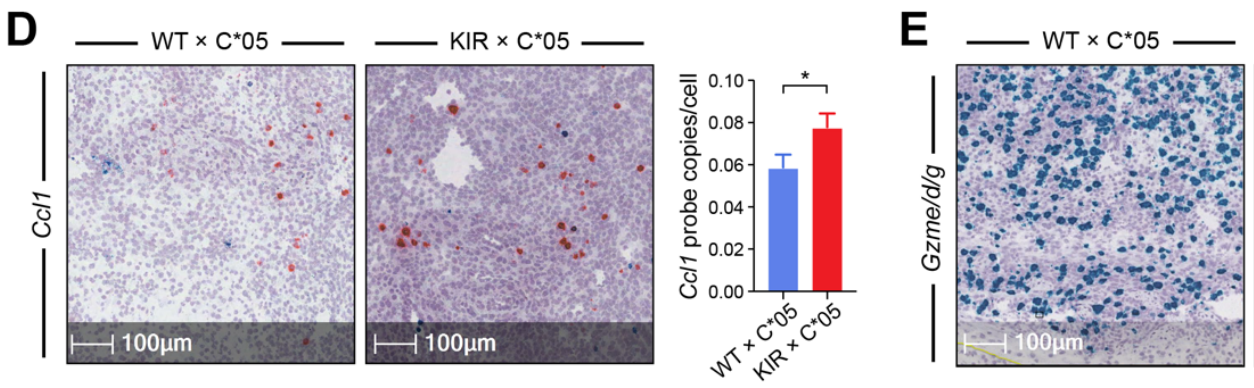
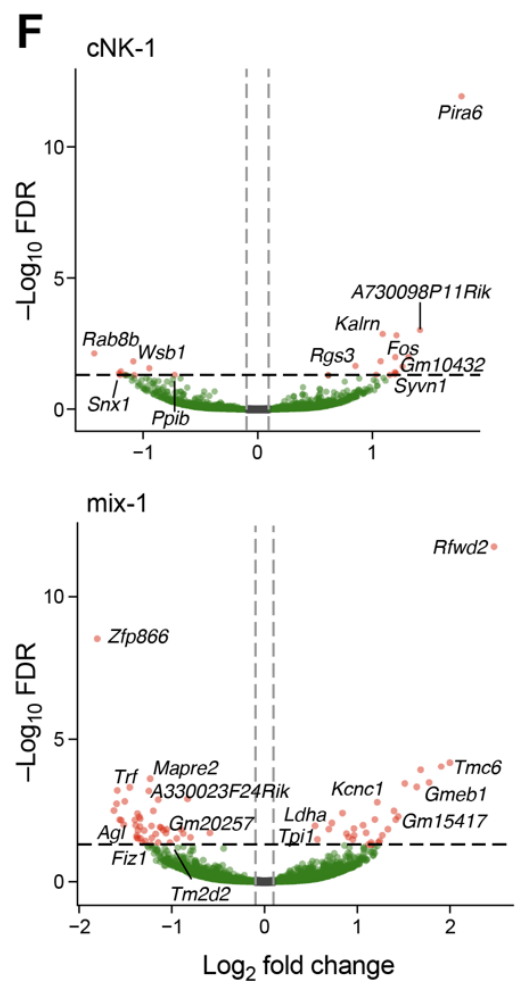
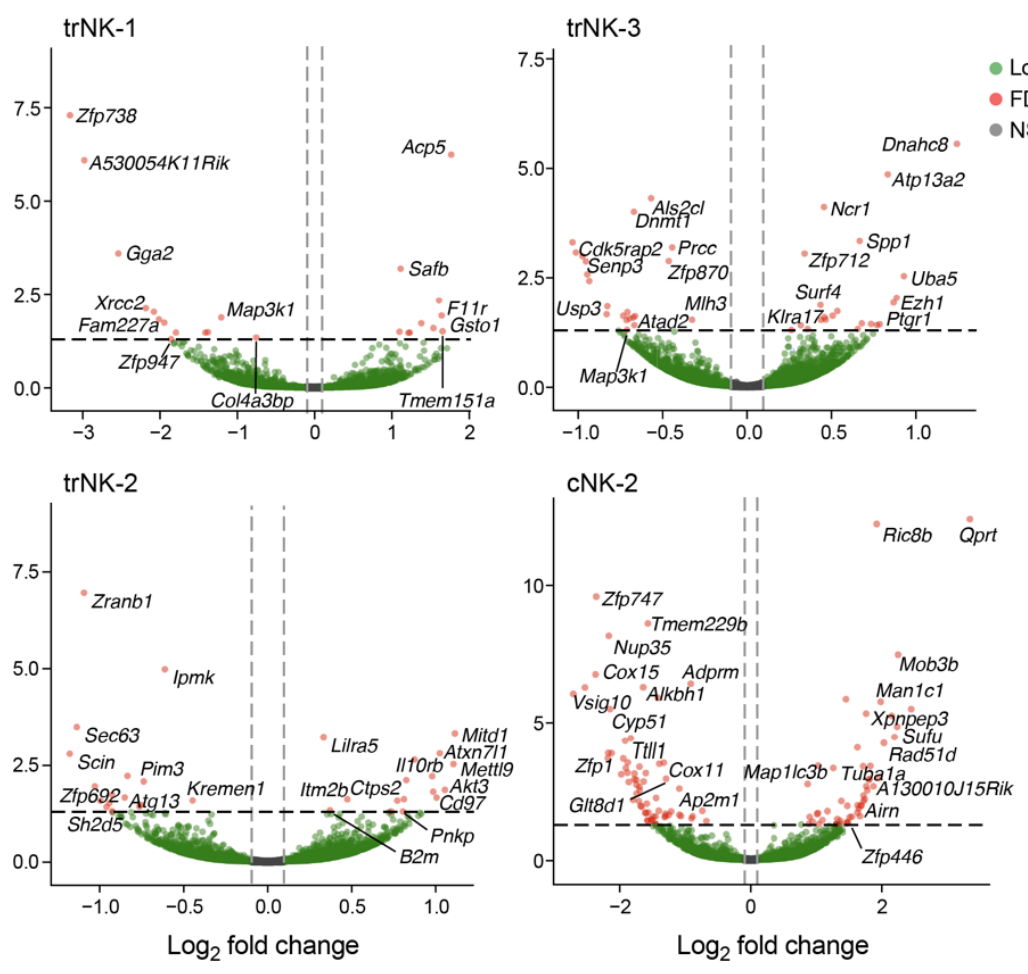

Figure 6
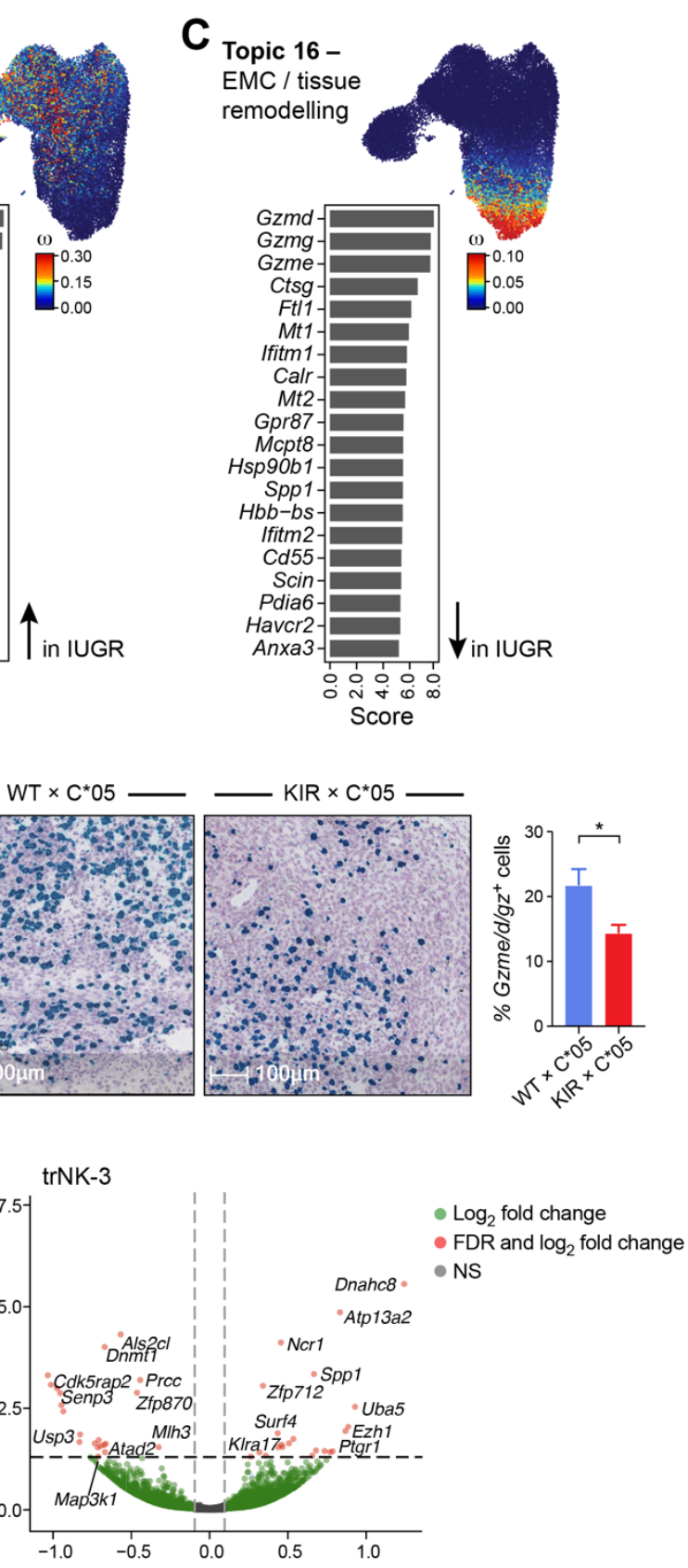
bioRxiv preprint doi: https://doi.org/10.1101/2021.03.26.437292; this version posted March 28, 2021. The copyright holder for this preprint (which was not certified by peer review) is the author/funder. All rights reserved. No reuse allowed without permission.

Figure 7

A

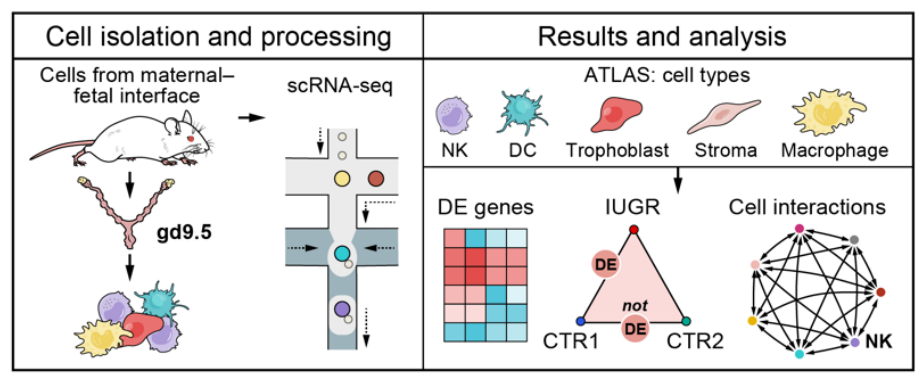

C
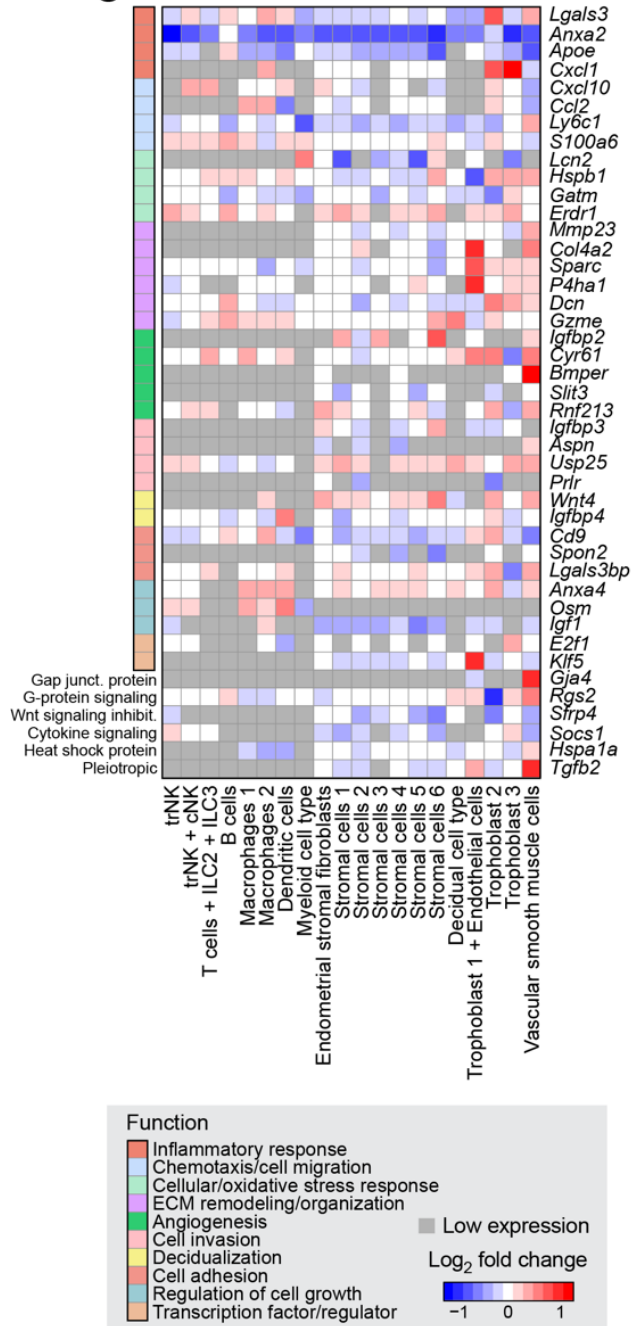

B
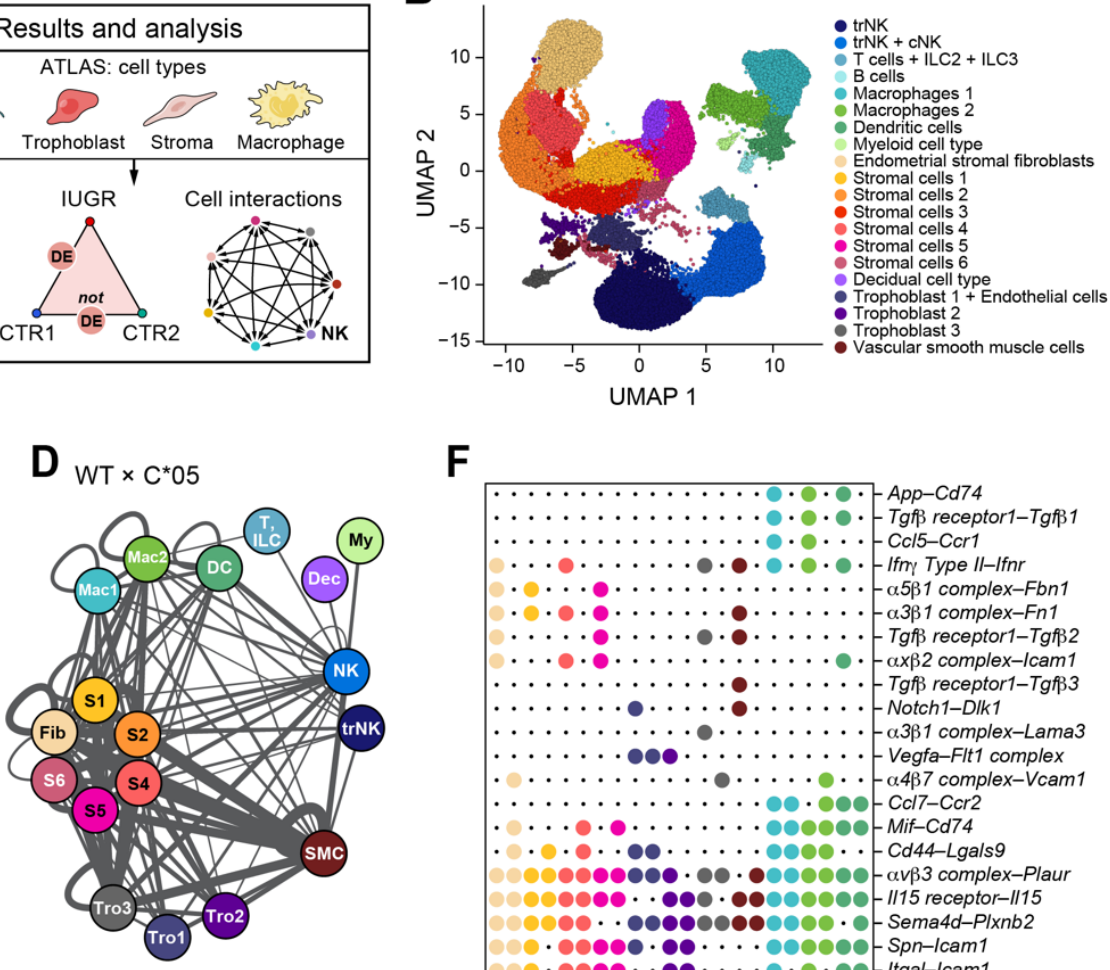

$\mathbf{E}_{\mathrm{KIR} \times \mathrm{C}^{*} 05}$

- Increased interactions

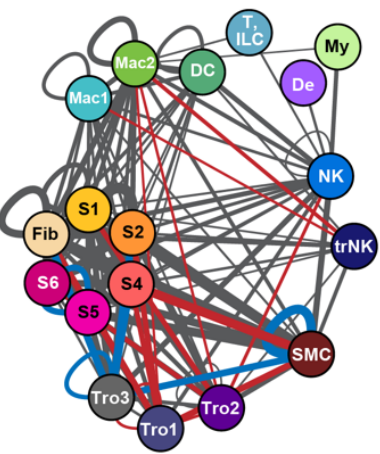

$\mathbf{F}$

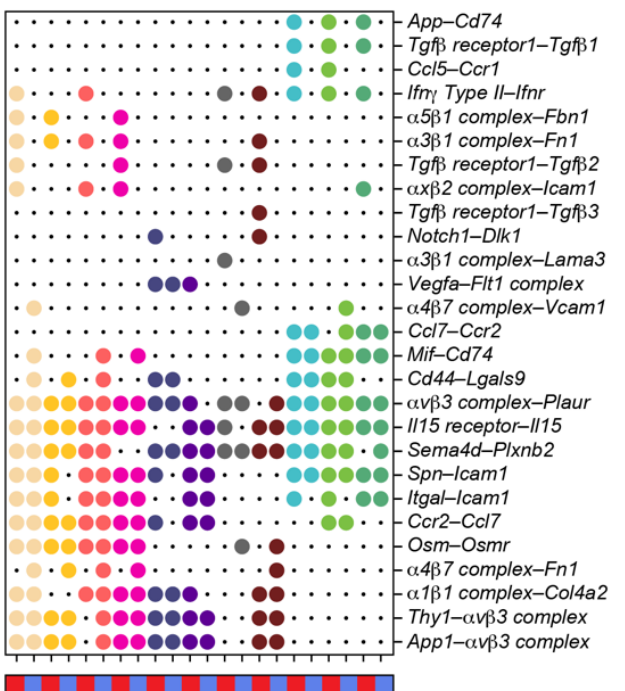

\begin{tabular}{|c|c|c|c|}
\hline \multicolumn{2}{|c|}{$\begin{array}{l}\text { Cell type } \\
\text { classification }\end{array}$} & \multirow{3}{*}{$\begin{array}{c}\text { Mating } \\
\text { groups } \\
=\mathrm{WT} \times \mathrm{C}^{*} 05 \\
\mathrm{KIR} \times \mathrm{C}^{*} 05\end{array}$} & \multirow{2}{*}{$\begin{array}{l}\text { Receptor-Ligand pair } \\
\text { (1) } \\
\text { Gene-Gene } \\
\text { (1) Found in NK cell } \\
\text { type cluster }\end{array}$} \\
\hline $\begin{array}{l}\text { Fib } \\
\text { S1 } \\
\text { S4 }\end{array}$ & $\begin{array}{l}\text { Tro3 } \\
- \text { MMC } \\
\text { Mac1 }\end{array}$ & & \\
\hline $\begin{array}{l}\text { S5 } \\
\text { Tro1 } \\
\text { Tro2 }\end{array}$ & $\begin{array}{l}\text { Mac2 } \\
\text { DC }\end{array}$ & & $\begin{array}{l}\text { (2) Found in interacting } \\
\text { non-NK cell type } \\
\text { cluster }\end{array}$ \\
\hline
\end{tabular}


Supp. Fig. 1

A

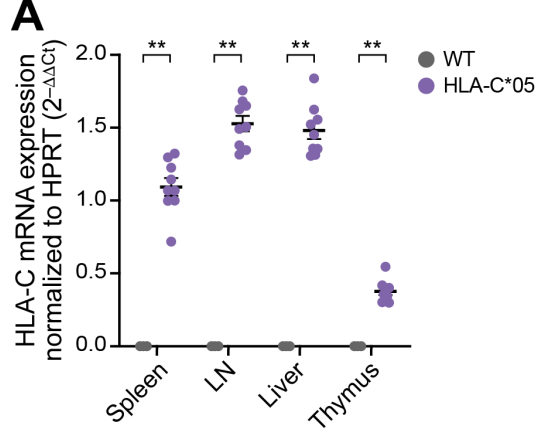

D

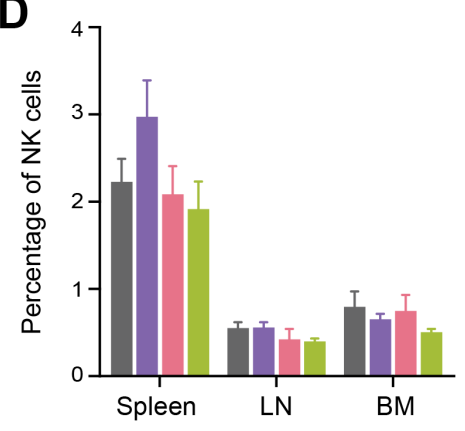

G

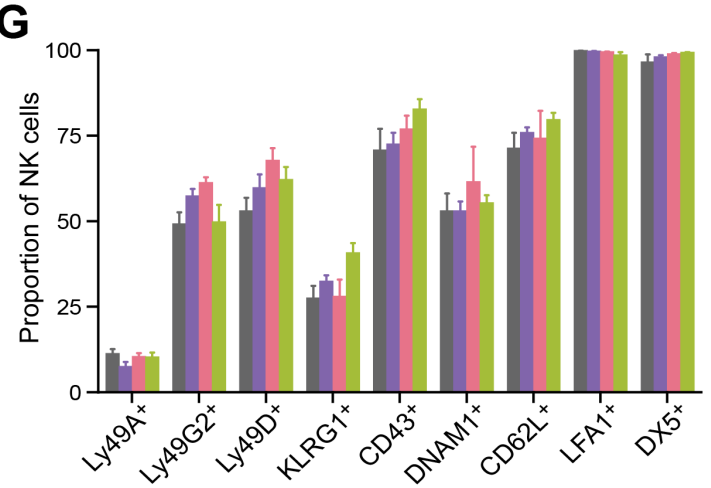

$\mathbf{J}$

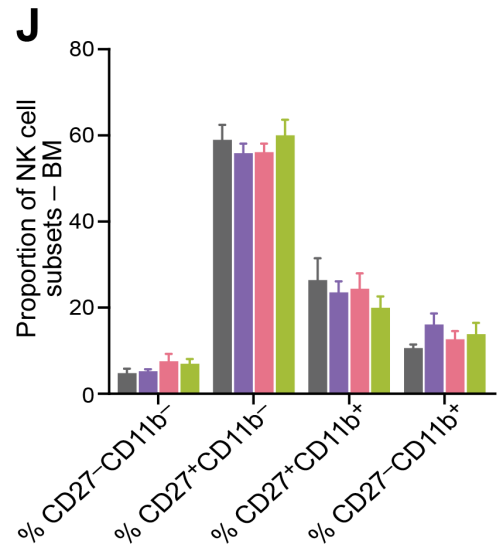

B

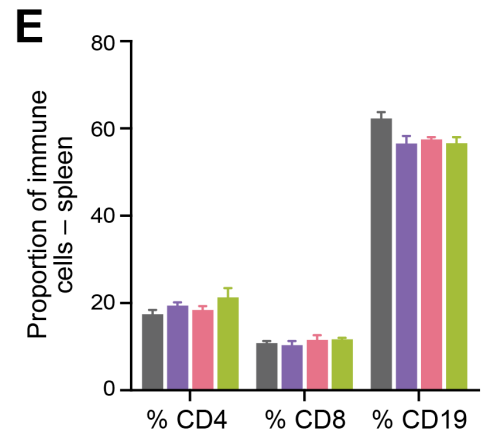

C
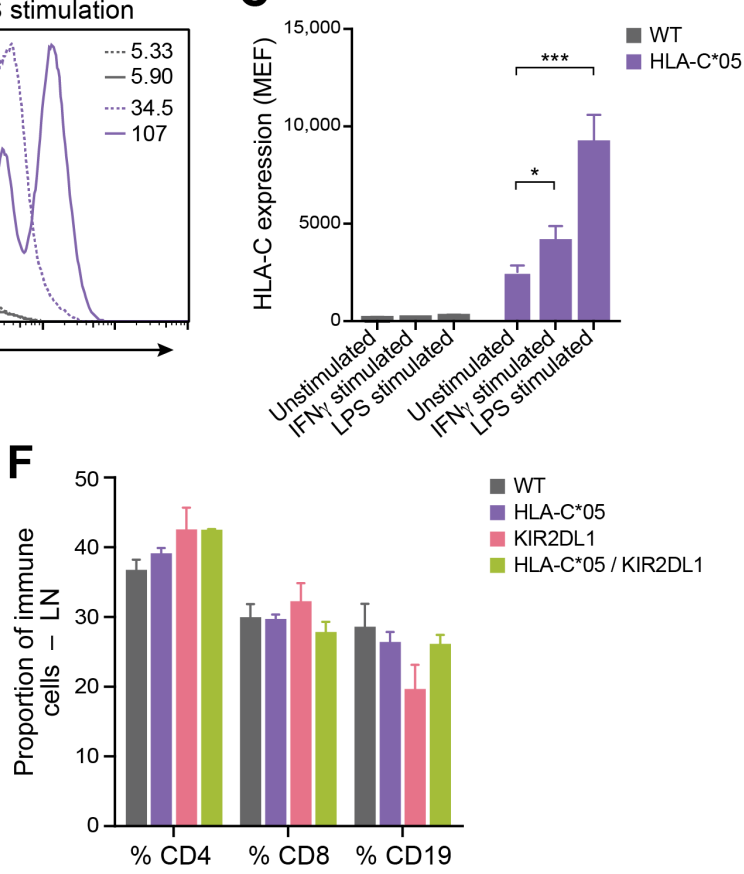

- WT

HLA-C*05

HLA-C*05 / KIR2DL1
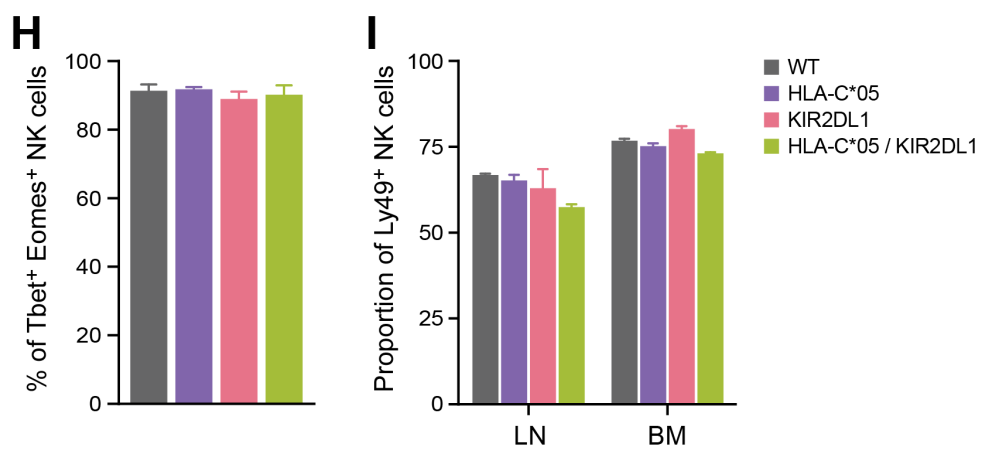

K

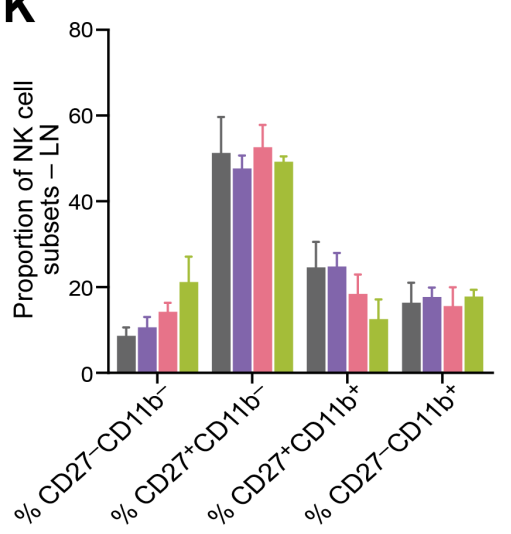

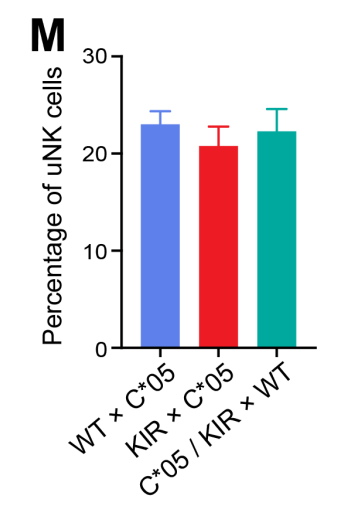

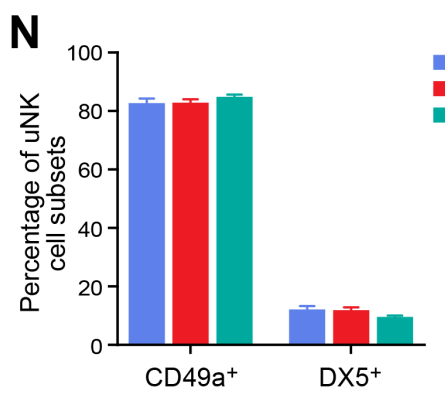

WT

- HLA-C*05

KIR2DL1

- HLA-C*05 / KIR2DL1

WT $\times C^{*} 05$

$\mathrm{KIR} \times \mathrm{C}^{*} 05$

$C^{*} 05 / \mathrm{KIR} \times W T$
L

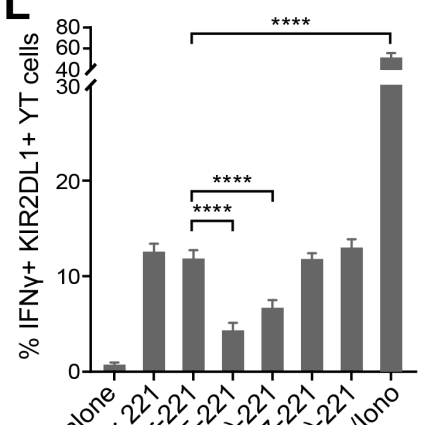

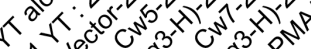


A

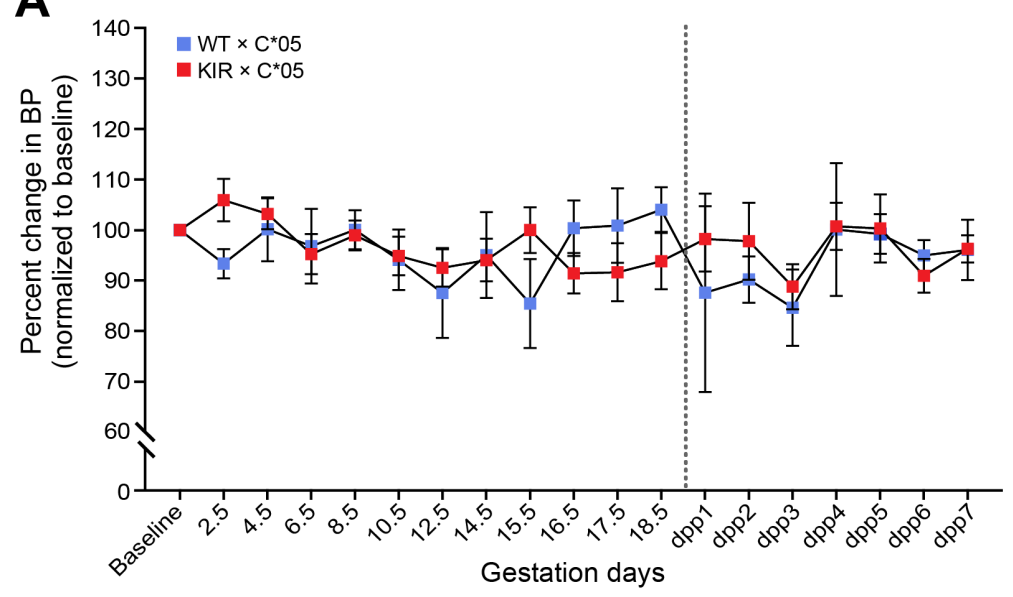

C

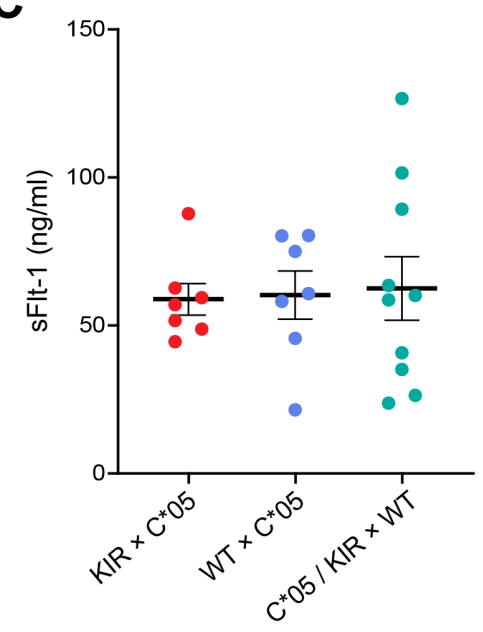

B
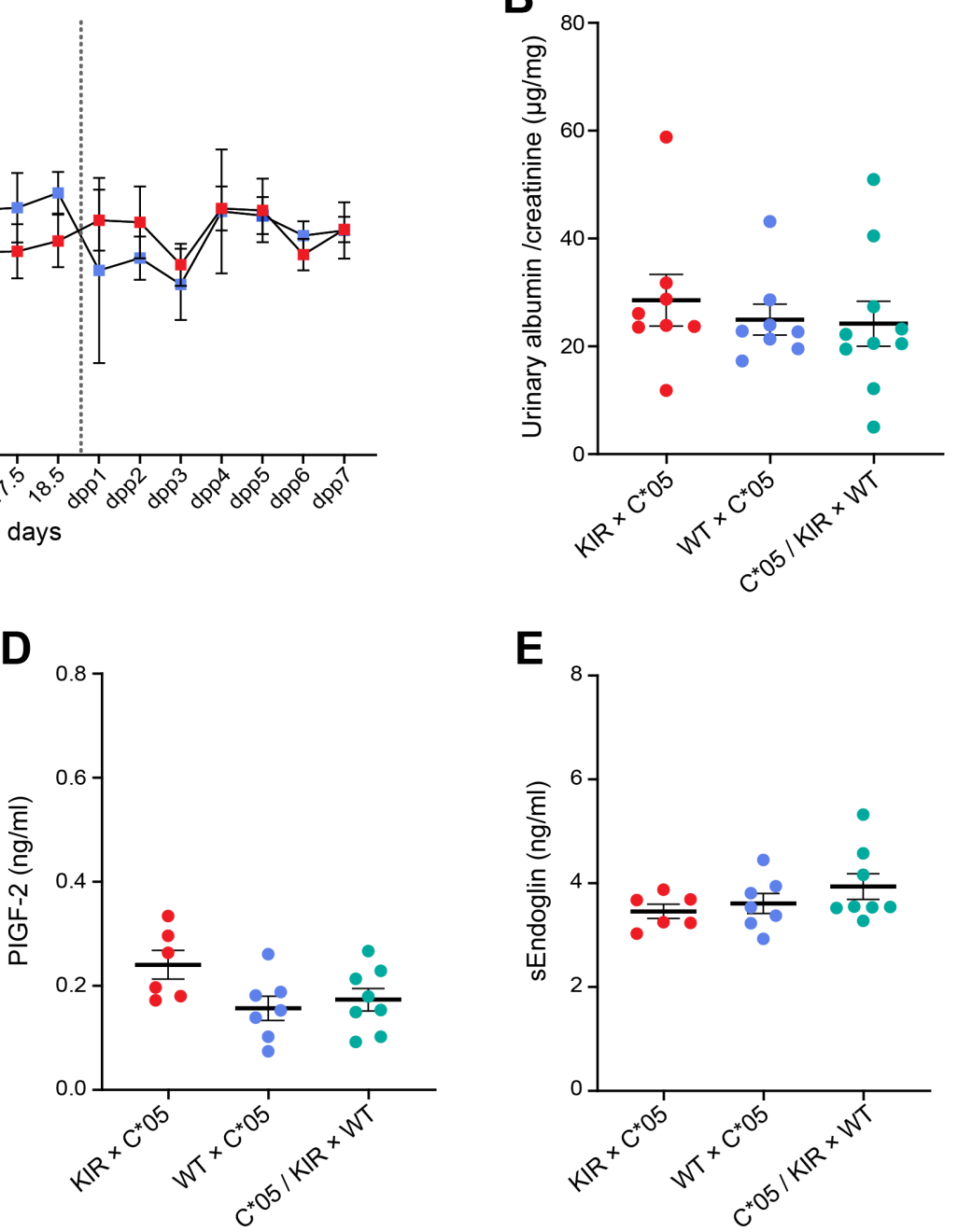

E

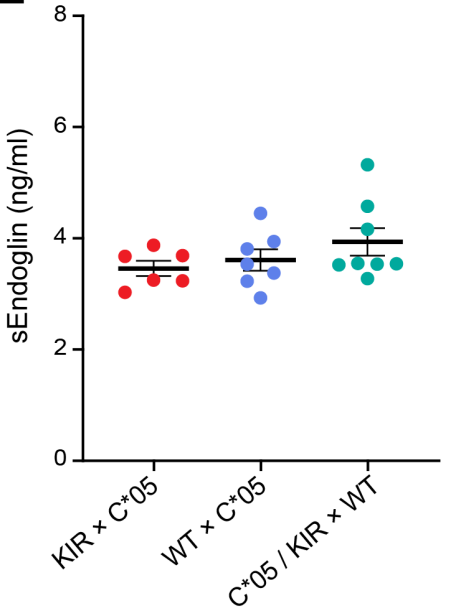




\section{Supp. Fig. 3}

A

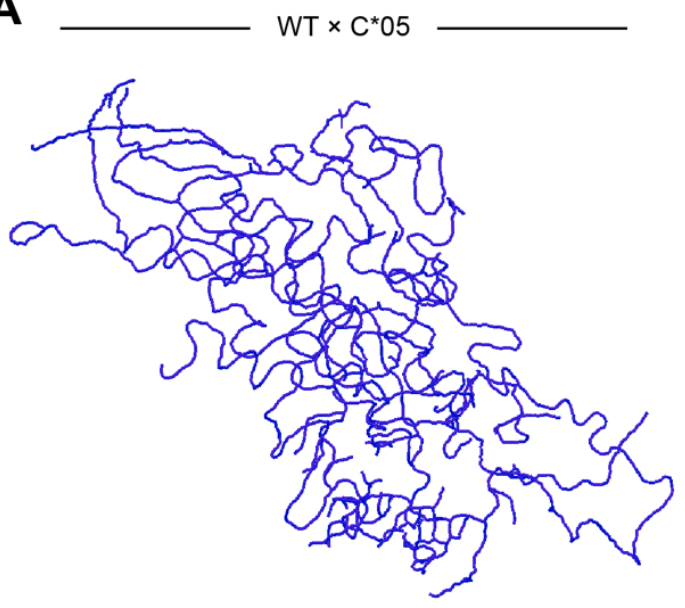

C

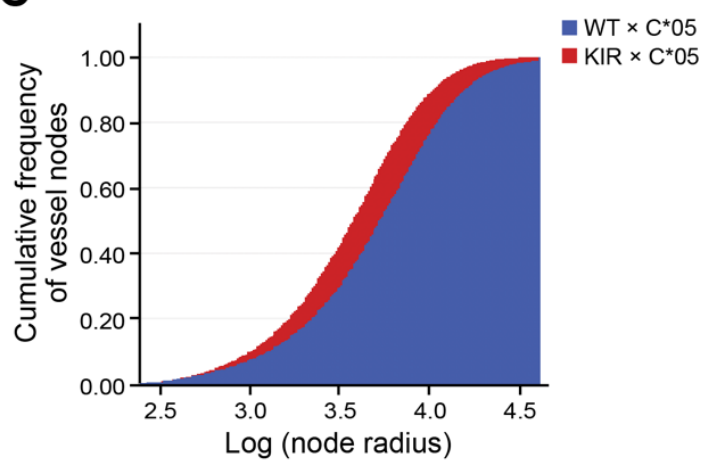

E

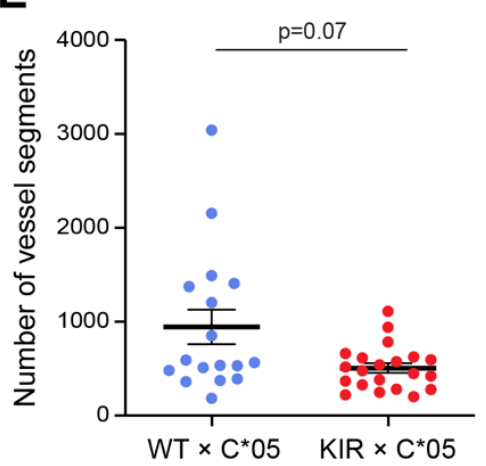

$\mathbf{F}$

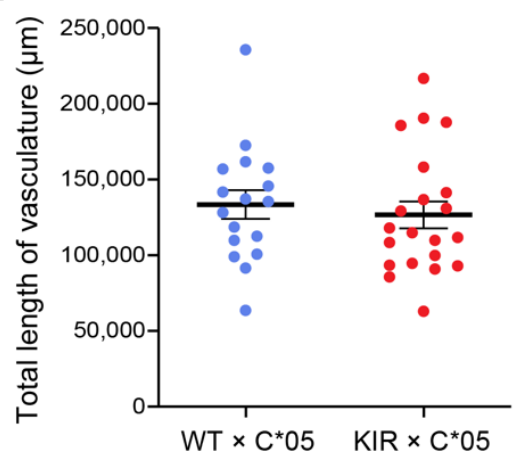

B

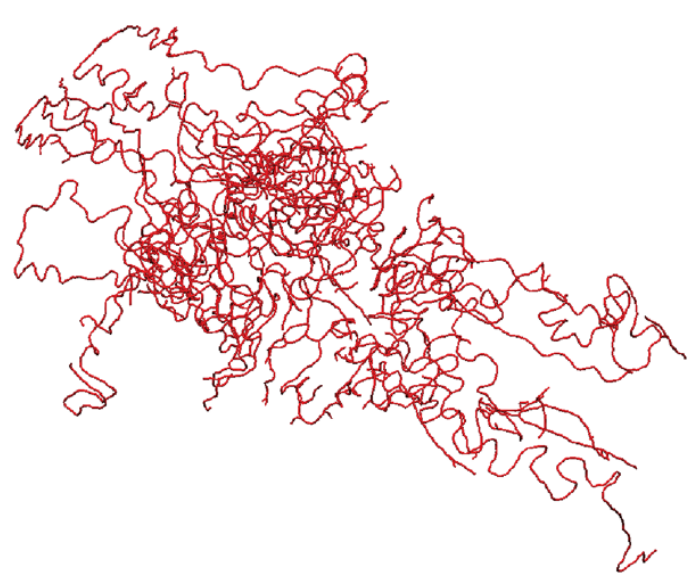

D

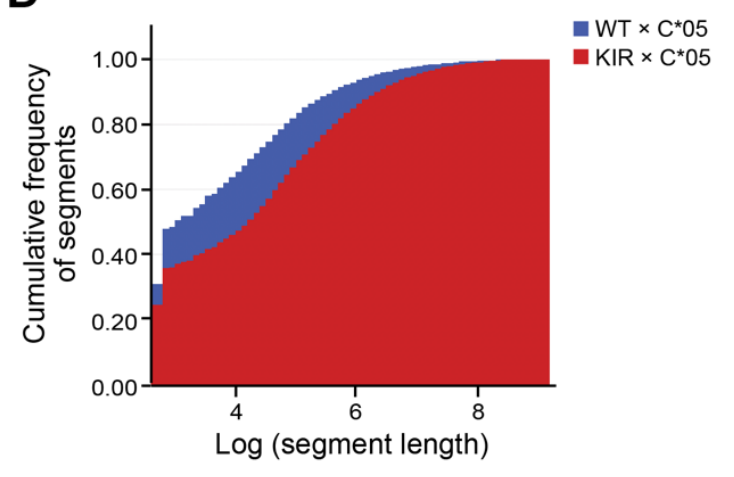

G

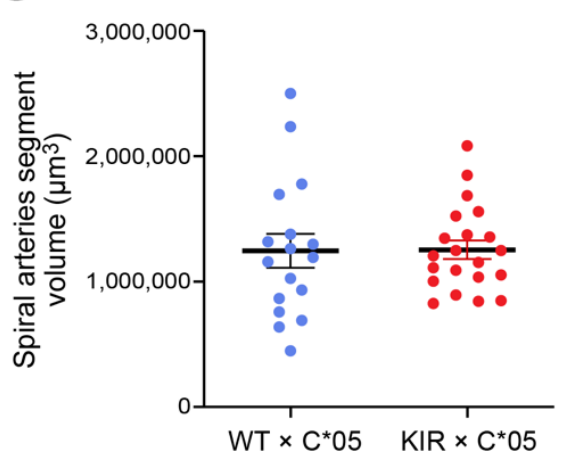




\section{Supp. Fig. 4}

A

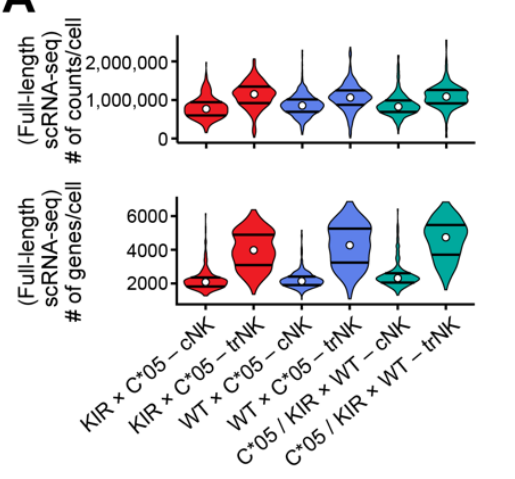

D

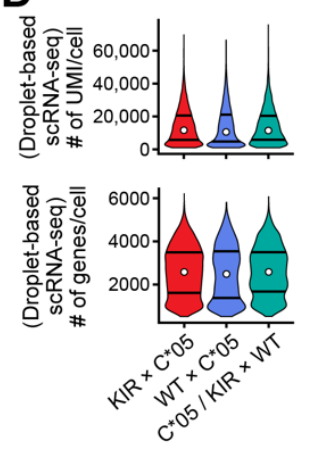

E

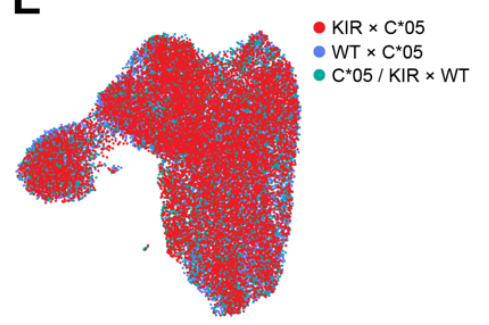

B

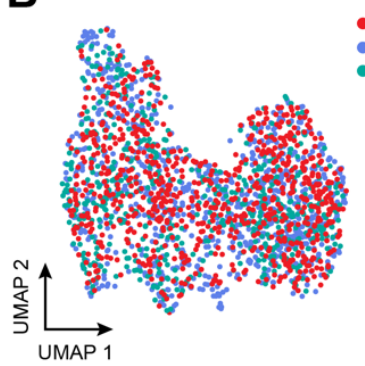

C

- KIR $\times \mathrm{C}^{*} 05$

- WT $\times \mathrm{C}^{*} 05$ $C^{*} 05 / \mathrm{KIR} \times$ WT

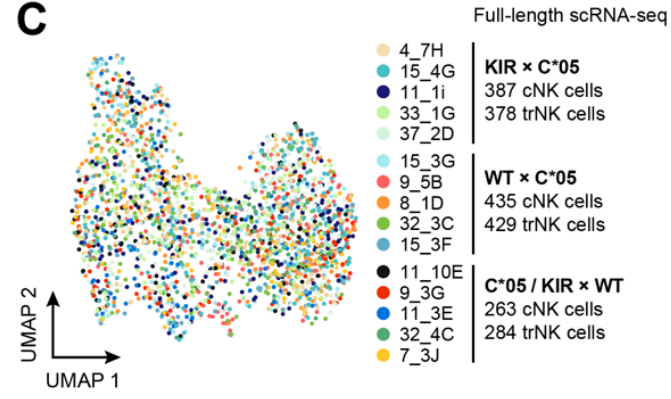

$\mathbf{F}$

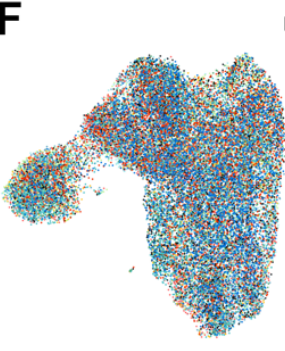

Droplet based scRNA-sec - 47_1P; 3505 cells - 45_3E; 3490 cells - 17_3D; 3803 cells - 43_1G; 3700 cells $43 \_1 \mathrm{H} ; 2925$ cells 46_4C; 2464 cells - 47_2D; 3126 cells - 47_1K; 1788 cells - 46_2E; 1977 cells -16_2H; 3369 cells

G

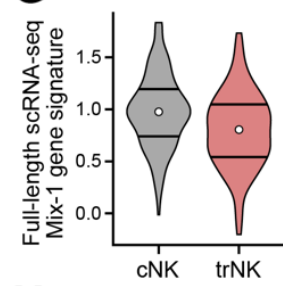

H

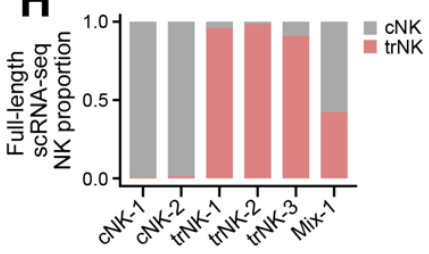

\section{I}

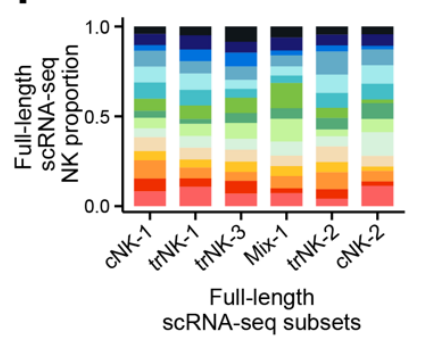

L

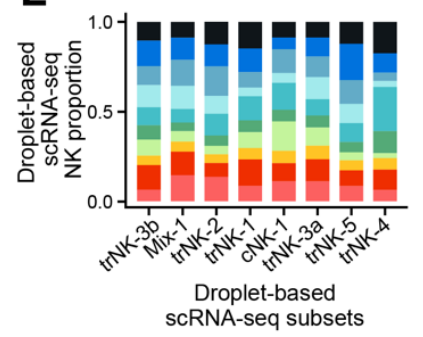

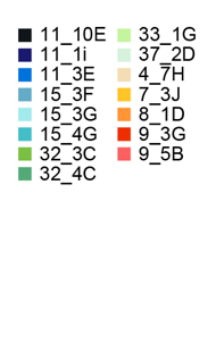

J
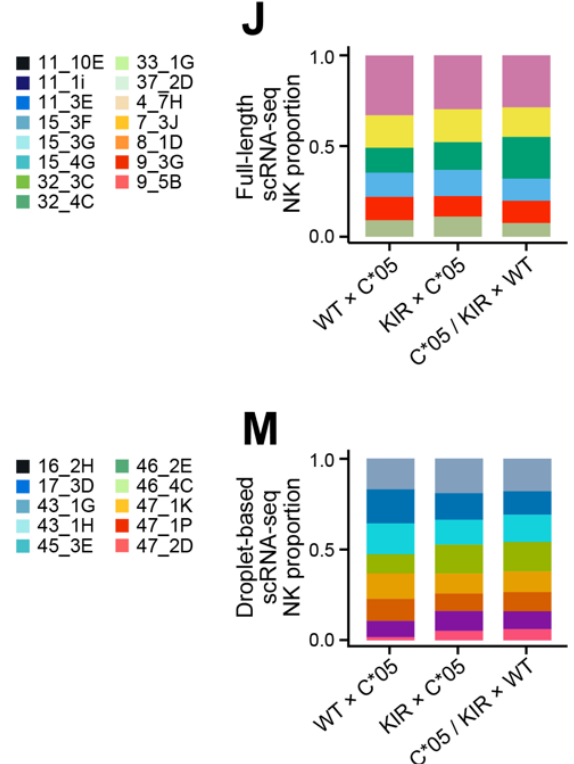

M

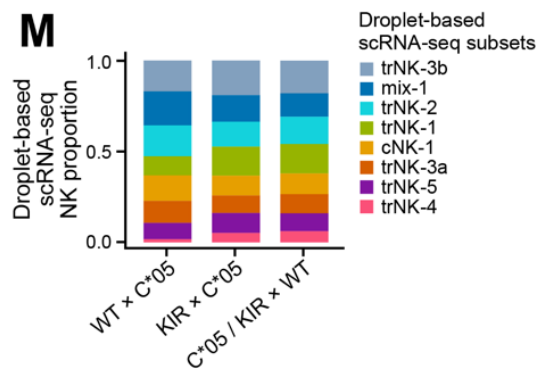

Full-length scRNA-seq subsets aNK-1

trNK-1

= mix-1 $^{2}=$
K
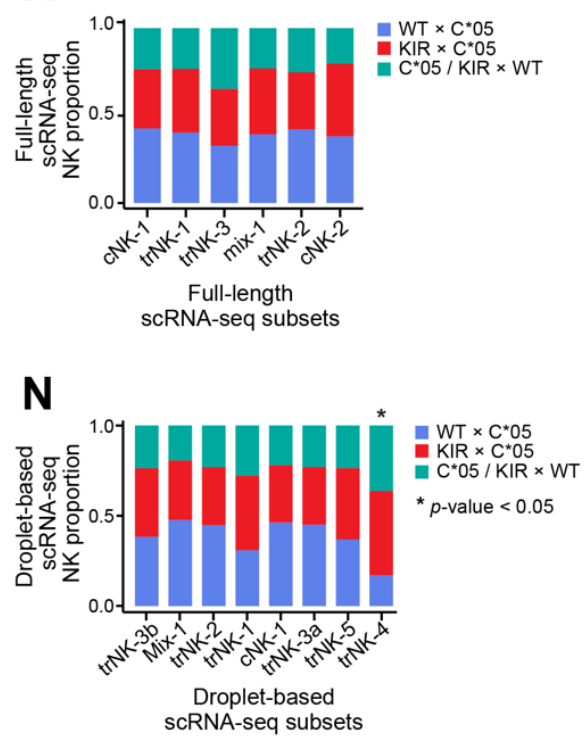


\section{Supp. Fig. 5}

A
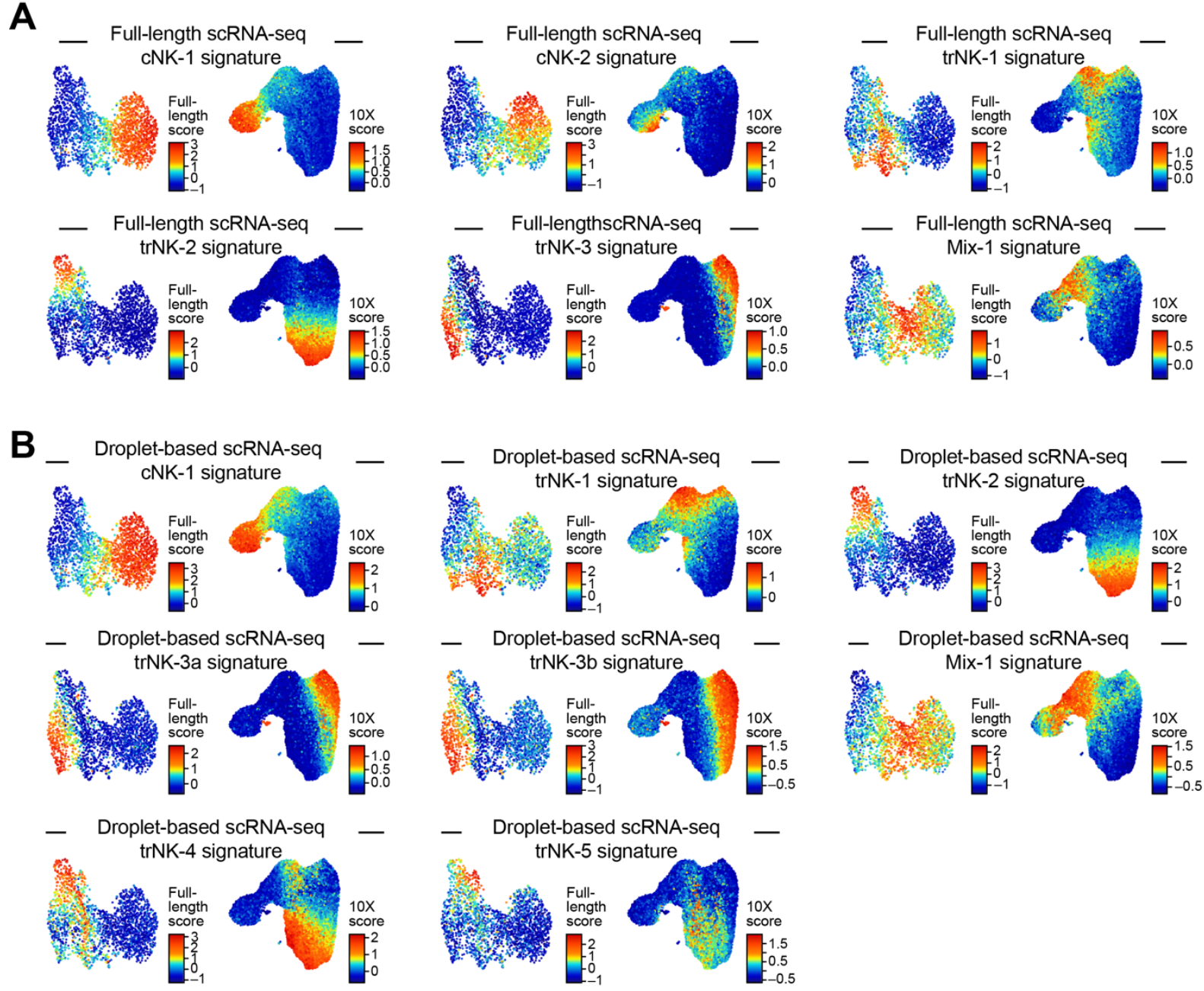

C
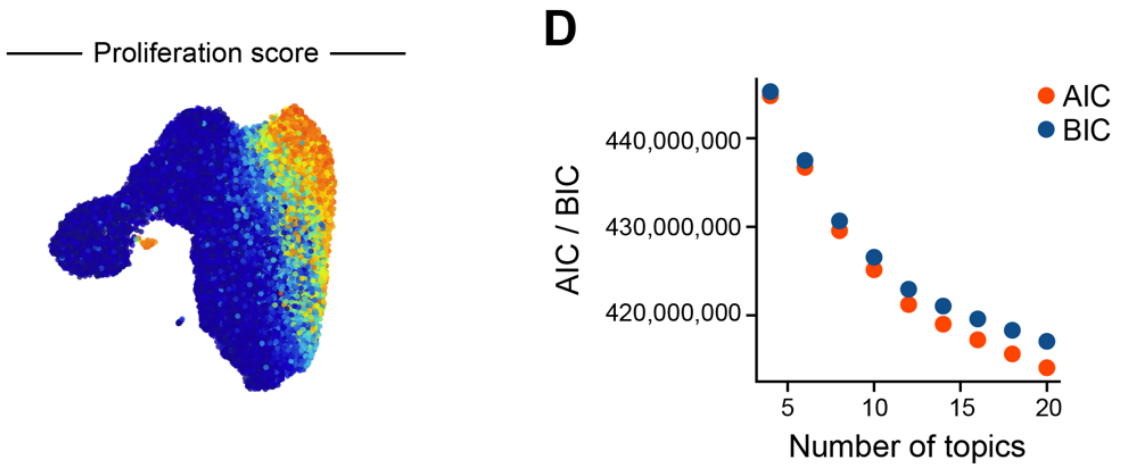


\section{Supp. Fig. 6}
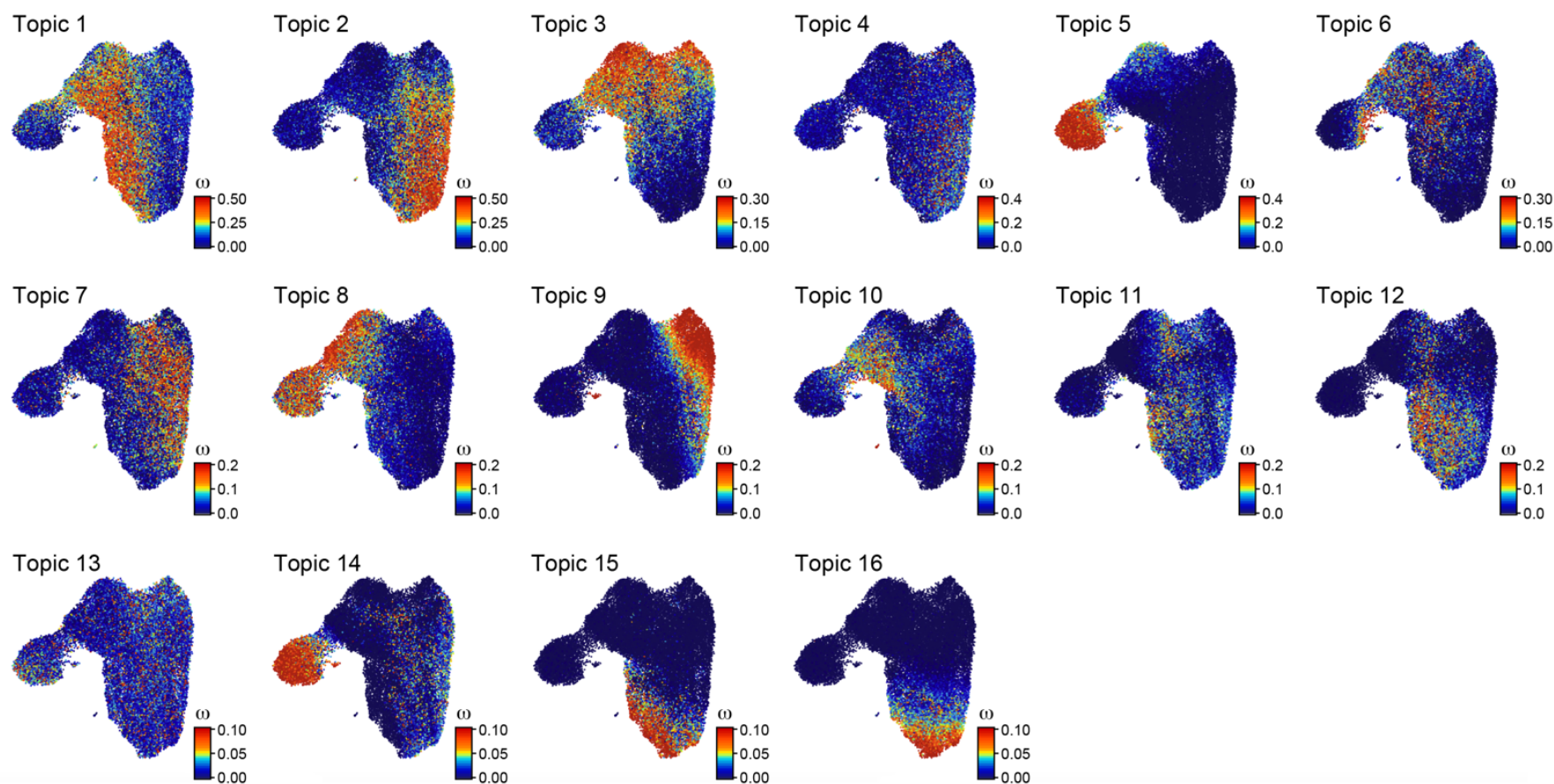


\section{Supp. Fig. 7}
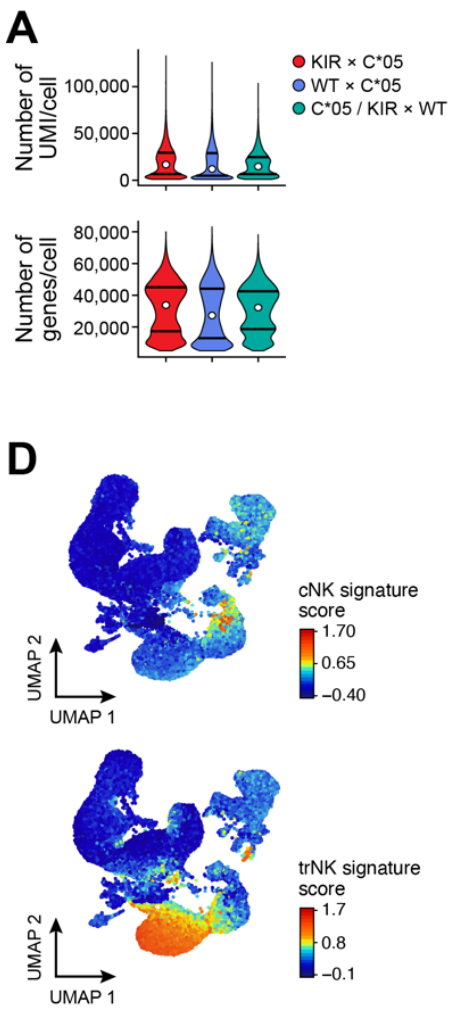

B

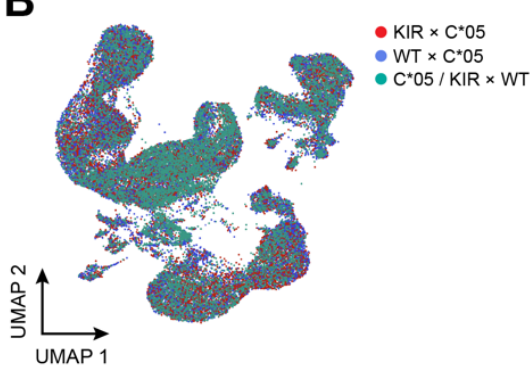

E

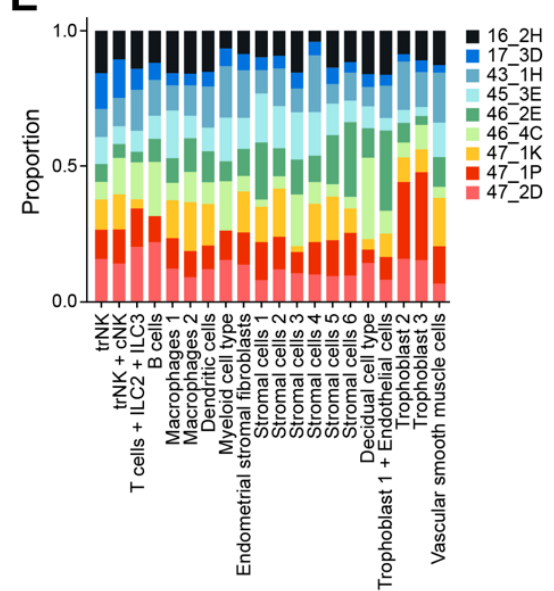

C

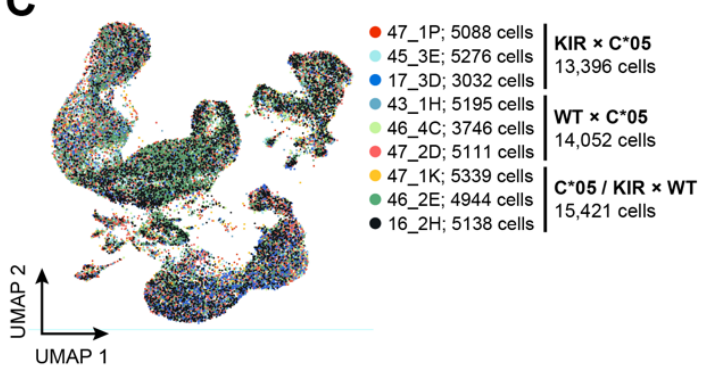

$\mathbf{F}$

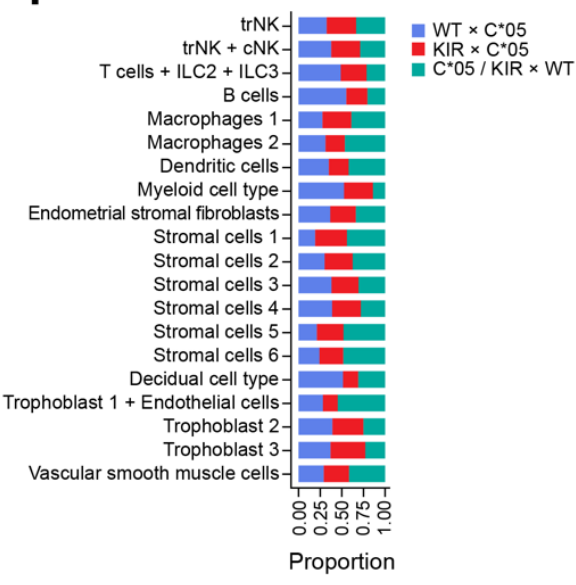

\section{G}

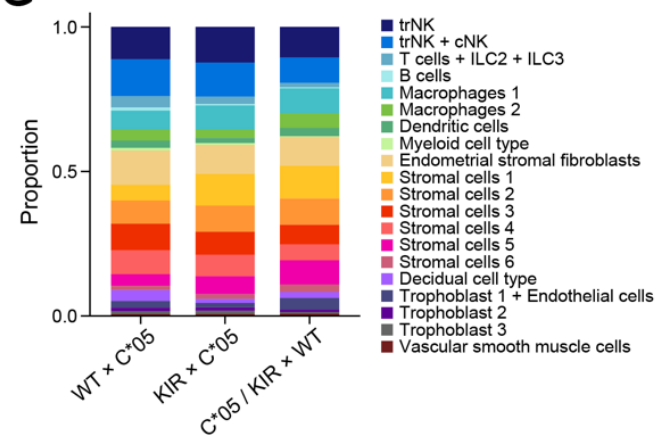

I

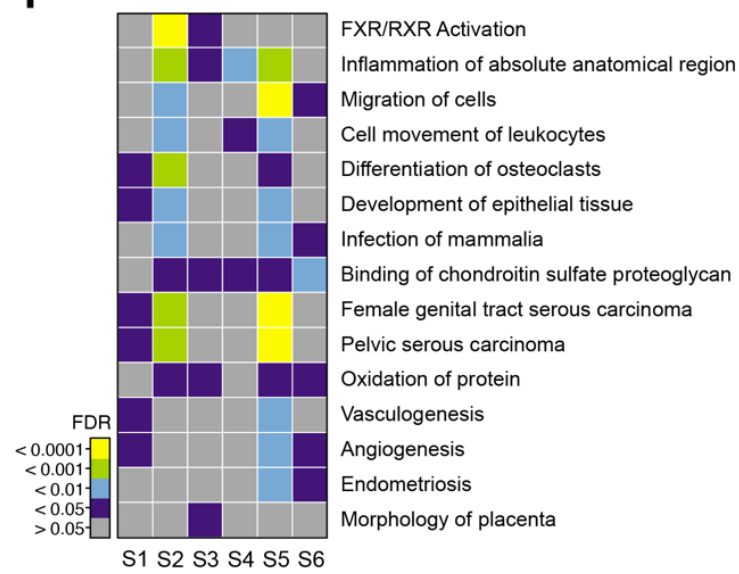

H
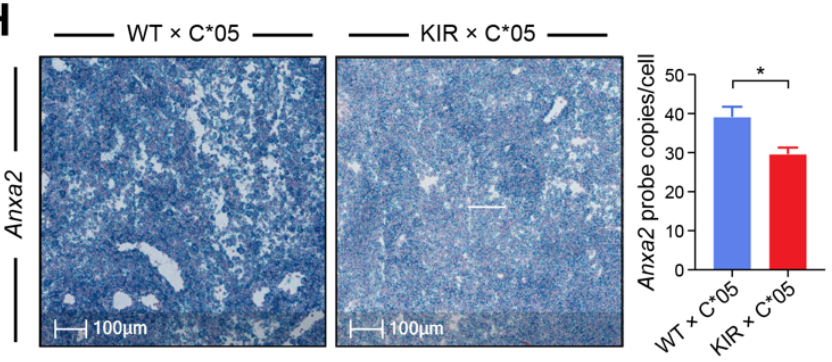

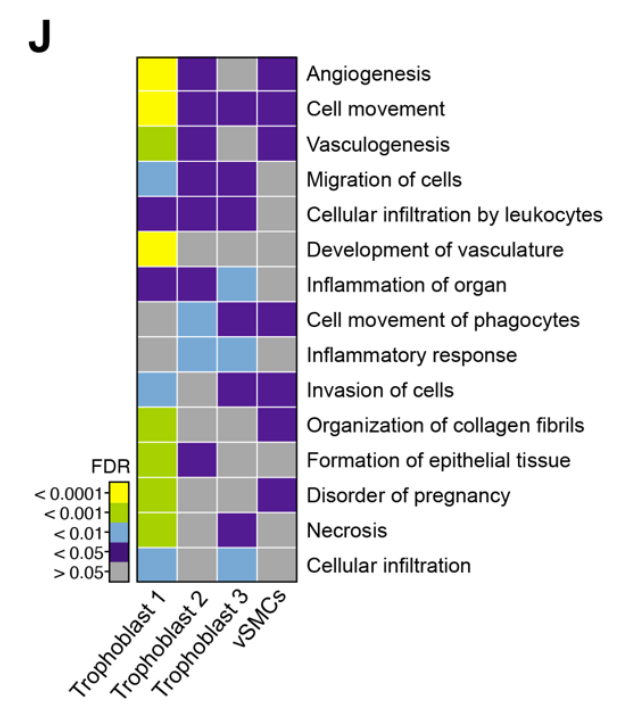

